$$
1915
$$

NorthDakota

Canada

Oregen 
3509

21

Vervan Bailey Warkington, D.P. 1834 Kelorama Rerad 1915 
May $2 \%, 1915$

Lft Wboshing $4815 \mathrm{Pm}$

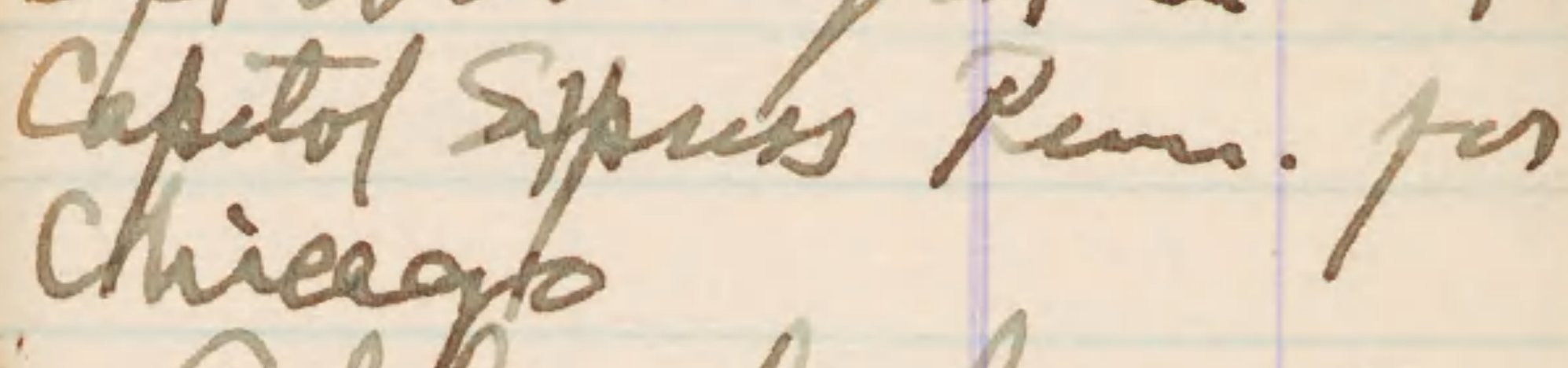

cold a d clanefs Vigitation unfurenx́

Mayso Orio

Chireago $2 \mathrm{cms}$

Thay $31^{\circ}$

Minueapolis $7.55 \mathrm{am}$

- Elkliber 10 am

June I Meradow Vale

Vimes.

Eiphrogus eyciostlolus 2

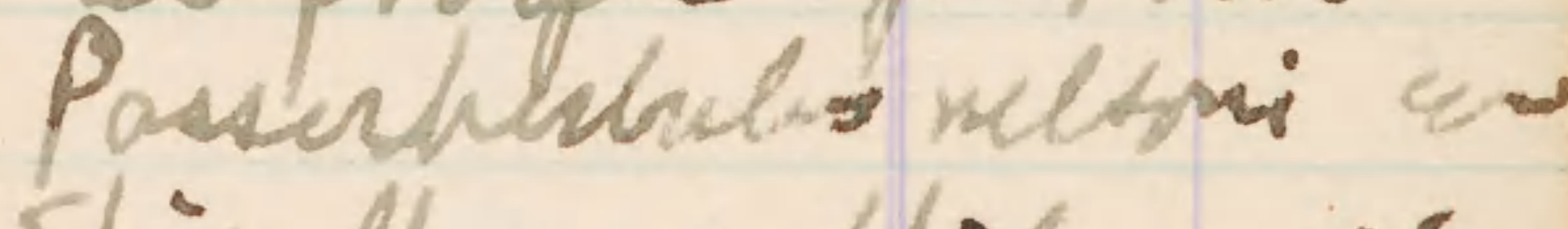

spigillo parlida

1. pusilla

- socentis. 


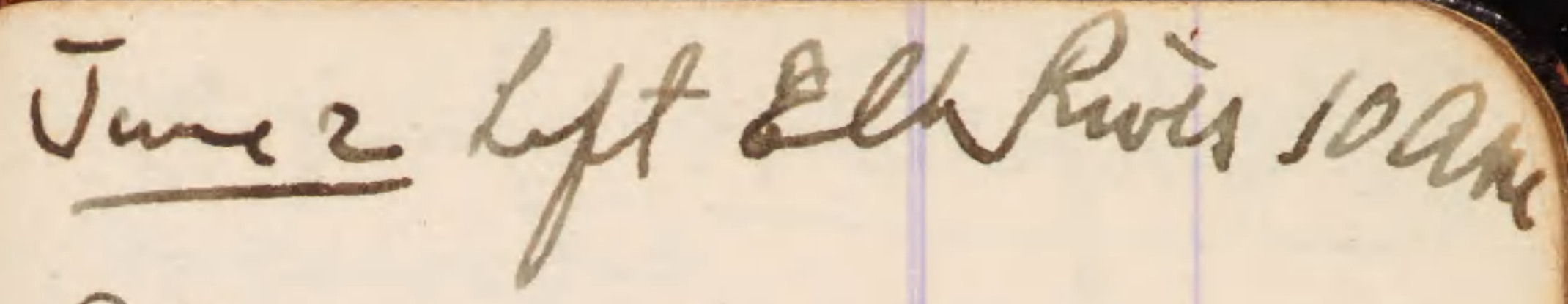

Stgples, chn peras Wadena

Battle Lale, a very lage s hounty it Labe

Fiqus folls

Walupes

GPM 
Wahpetan fur is. Drjobots parasas , oprea,

Iuliea

. Iymponnghere

- Inaidura

- Aryle alayon

- Agrains

- Nolothras

- Quicseahes annero

- Dolijelama

- Sturmella pregucta

- Letrias qabrula

- Zyramino Tyanoso vistisolis

- Myjochanes ivenas apes

- Enjpidernax

Mulanezpos

- Sitta

- Ravelucatid porfeal

- Whearciga

- Areedita.

- Ammoroliarmas

Cotrermeculas:

- Prapure truanig

- Hirando

- Pettochilion

- Cyar acita
1 Traglodytes

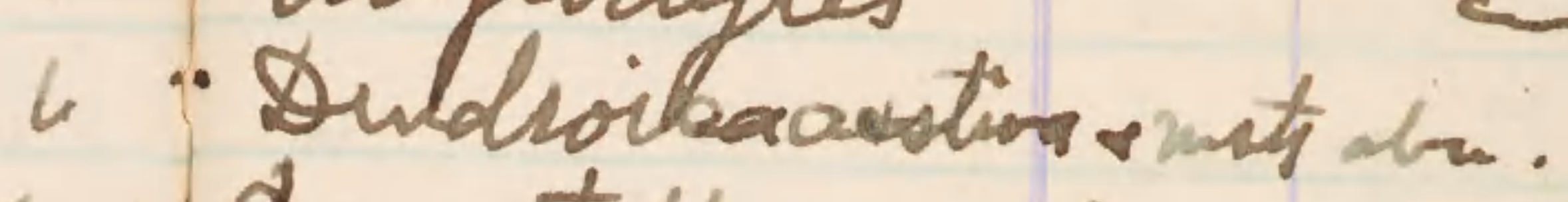

co Dumatilla candinasis C.

- Robir r matíg

- Sialia

- Cocenzus

- Colofar

* Amplila cudoram

"Dpyelus

- Gedtas

- Astragalins

- Spigilla socialos 1

w. Comns pállida

$3.7 y a s t m a$

$a \mu n$

a

a

c-

列

wo

.Vireosir y.

Vires gilons

- ¿1 alivacitas

“ Ripacia vinsts

sttlgadoptryx ? a fw

- Evechista ciodis I

woiciens

" Buto platyptrans

- Cusípitis ulo

- pus hisachas ceri tro

- stillsedostras

2

a

a fow

I

1

c

*

;

$$
1
$$

an

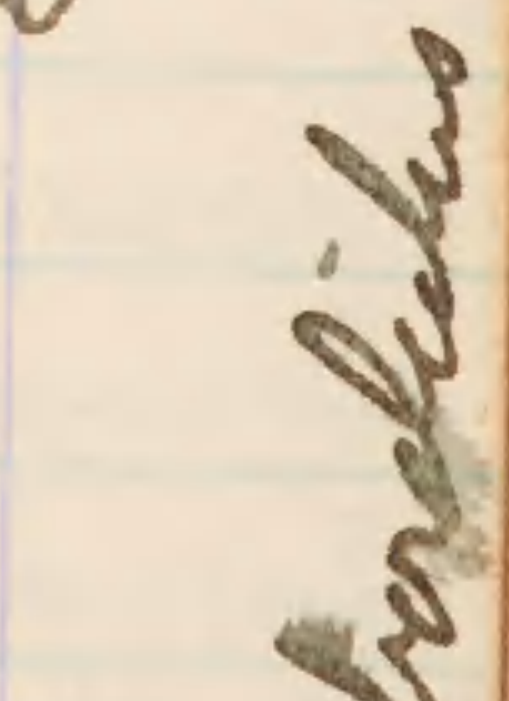

l

1

2 
Malpiton

uhuns

Nequndo

Trations

Quepens marocaypa a/s

Salix z

Papulus, notuation, or

Primis nelanorearpa don

Cratigus?

Prenuns wervensos ator

Cermus

Rrbes, flode,

Vitis

Menarperma?

C.eltis, fiegtres, con

Vifarrume apow

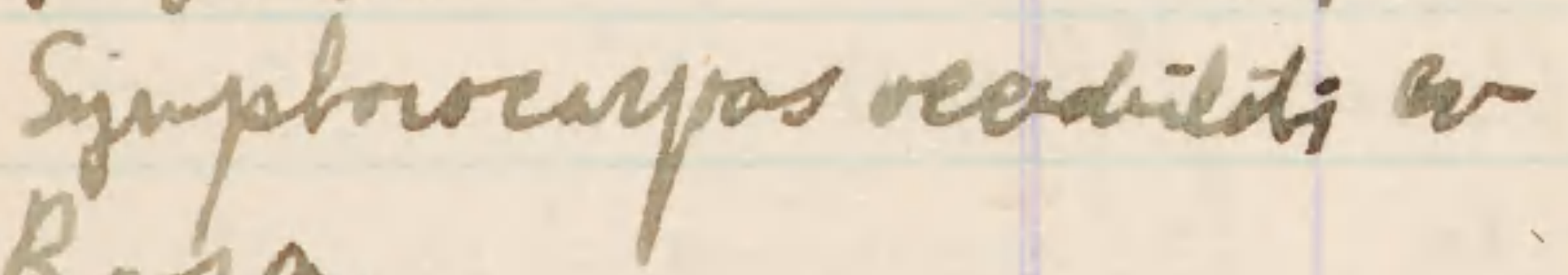

Roca

Grasulacia.

Poosaled argophylla

Anemons chadusis

Artinisian ludorration 
Watpetos. Jus-4.

Juich To whitiRadigpm

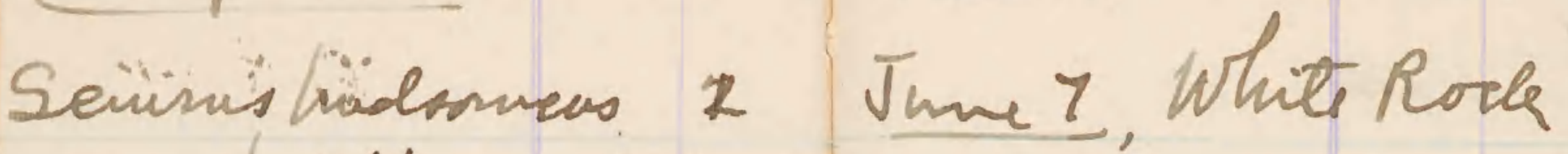

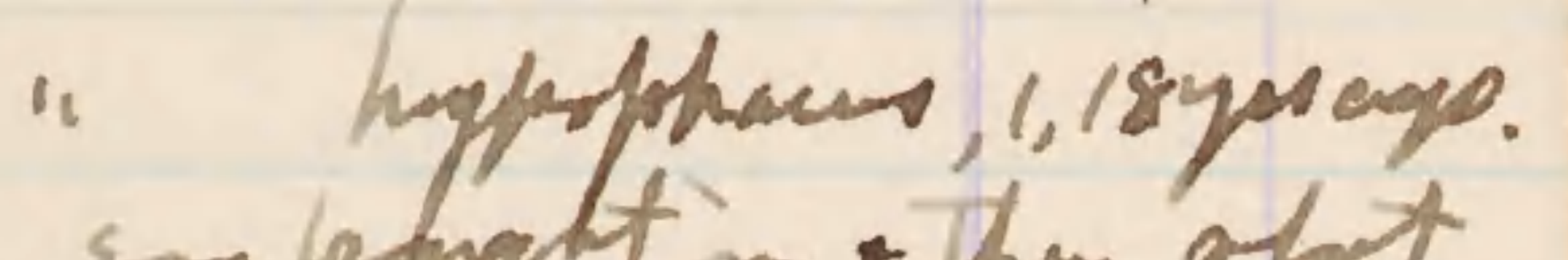

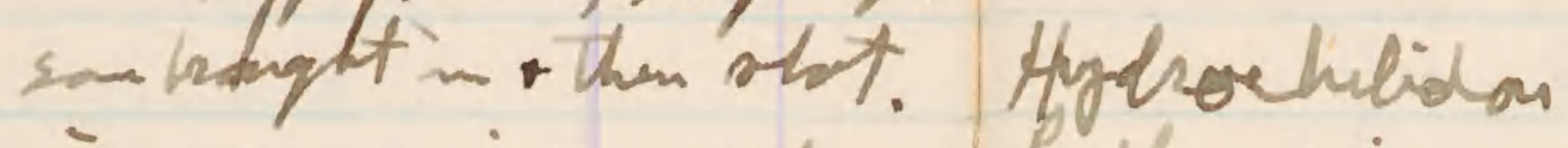

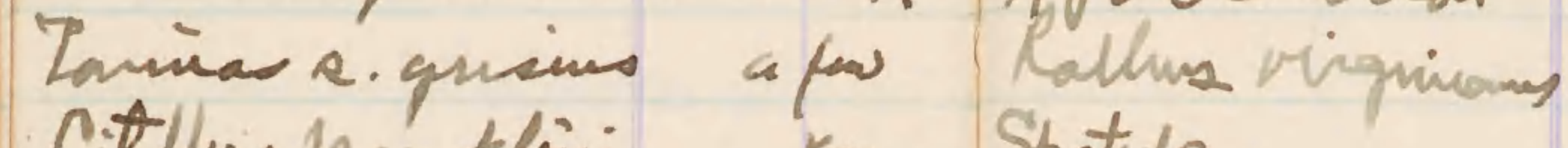
Cittlus pin klini zo Spatula

1. 1Elineatos is Xanthreaphalos

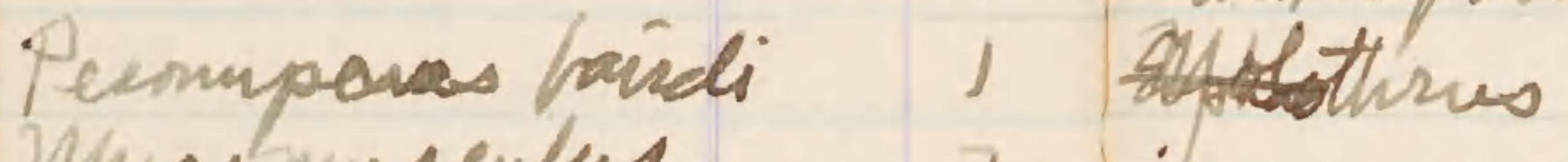
Mhararmentus Miesshes dew adi en

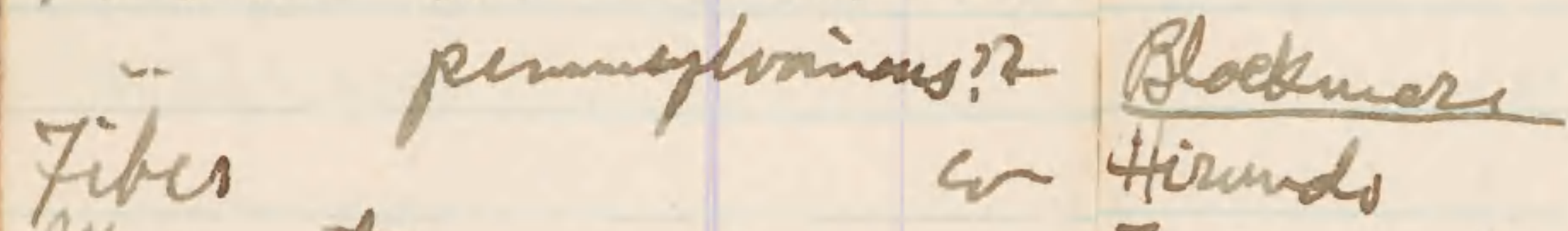

Maswota

quomys

i Treedyinta bieder rinest 5 coge.

- Strot eand aol +5 yg.

- Cyanvitta

sytirlagubs indis

is Stumella.

Pupesrugalis afes Cotmmentus taind

Proveyou

- Aurgolians sodudadium

Faxidea

Miphites

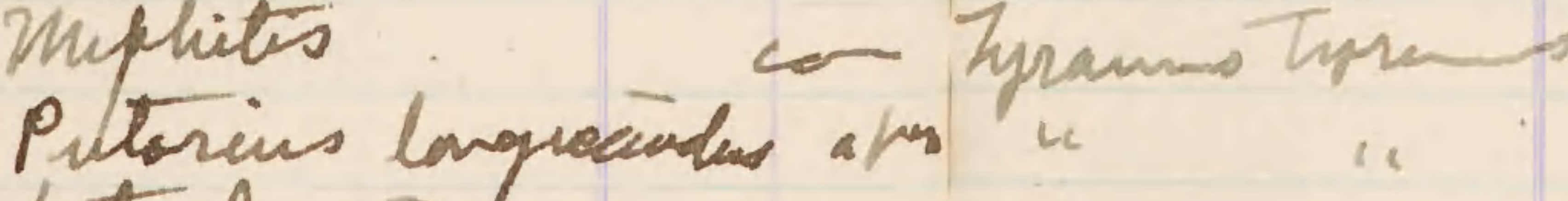
Lutrola visan

Blarivalurinecunda c-

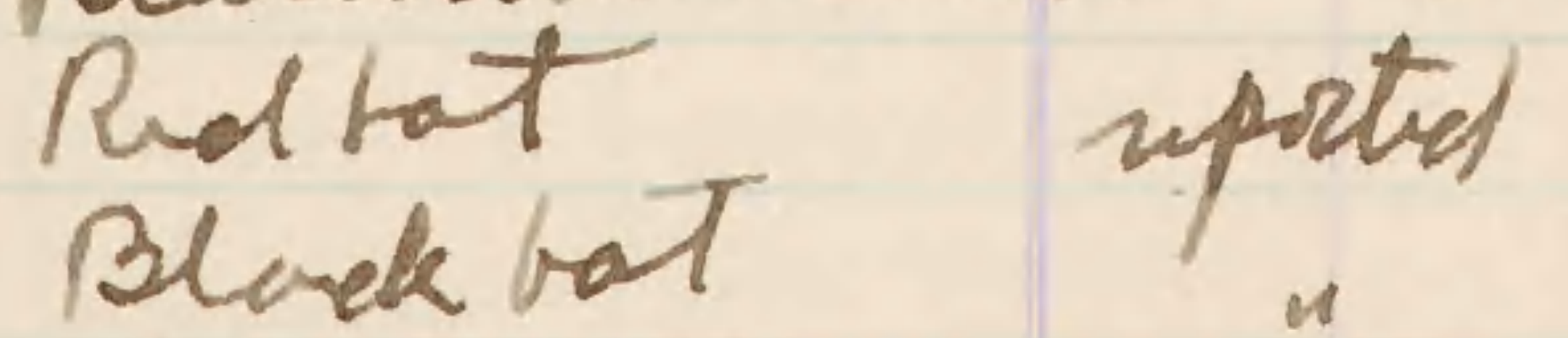

Hoarybor 


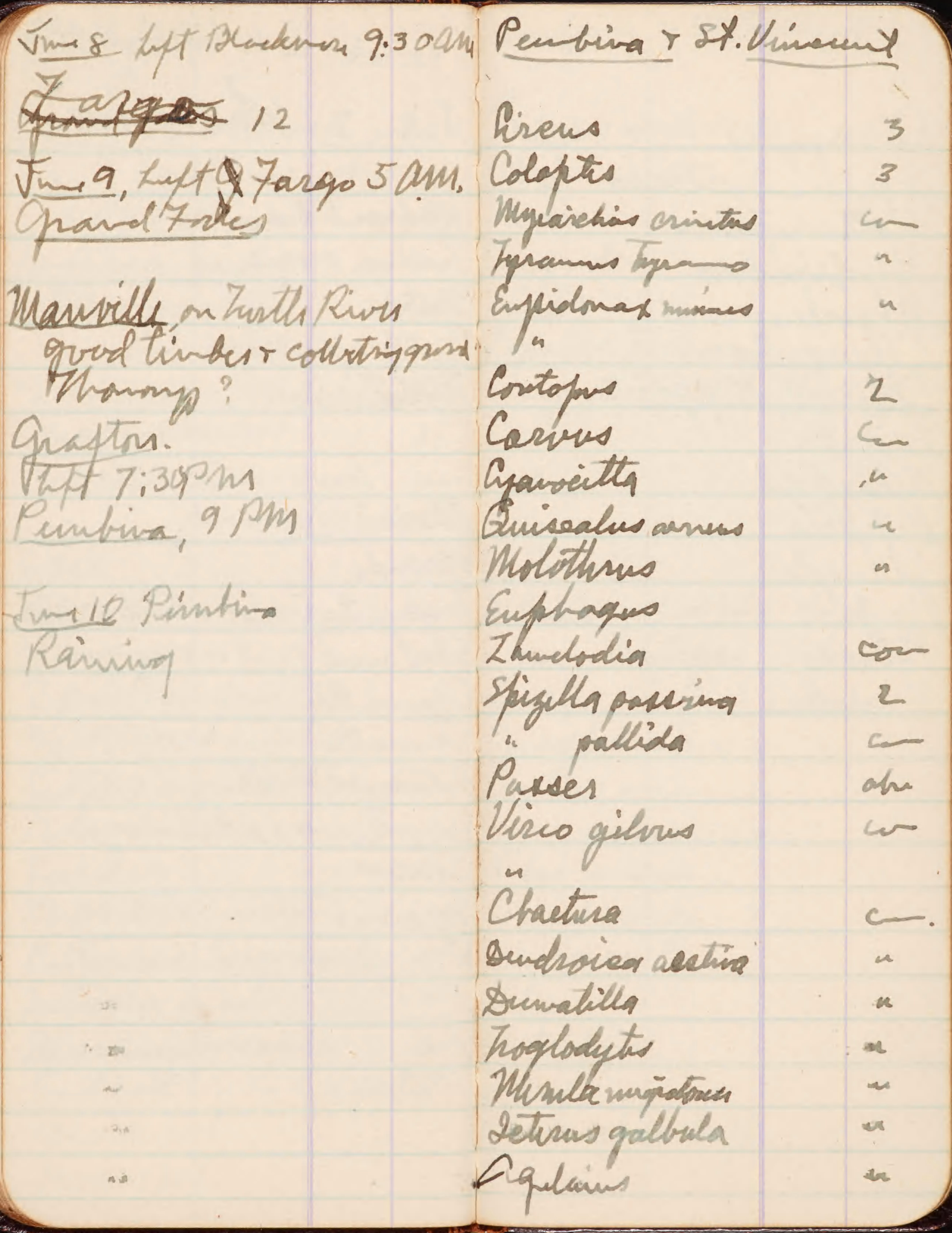


Putind \& St. Finérent

Coeeyzus

Typans ustivechy

astragalims

Hydeplubitas

Oyjuems

Chardiles

sturnallo snoghas

Poveritio

Melarpiga

Riparia

thimglo

amplis

Pedhocits corring

Zemaidma afew Onersus maceocerpo

an Hhuns areveana

- Trapinus

3 Muquado

con tilia.

1 Populus turubiales

an a stlowoods, introdad,

- Falix

" "

- Osctiqus

hased Viburmen opesephin

an " butage

3 Corrus

Corylas anineanas

Prumus arincaso

¿c molarreapa

Rifes arevecuna

Symptoreagpens

Vitis

Aupulopsis

Inpue

anmlanchies alisplin 


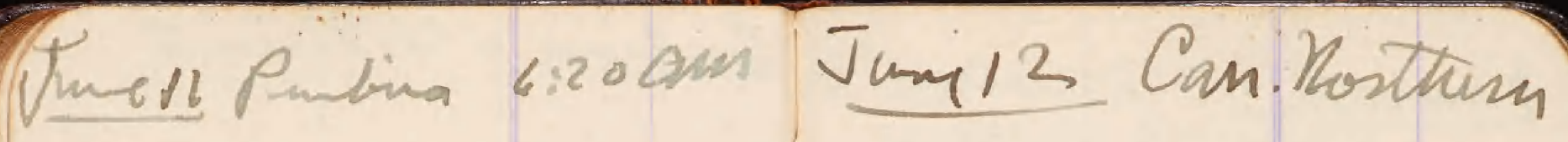

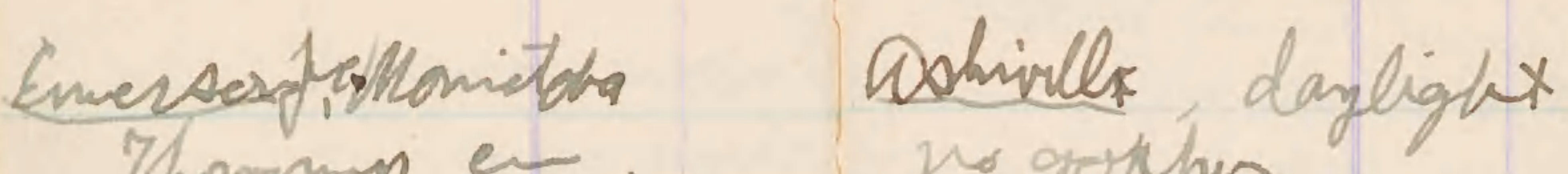

Thrming en.

uo go thes

Disaveltes as

Massis

shurdin

Riplins cosa. fint

no gophriniels

stagathe

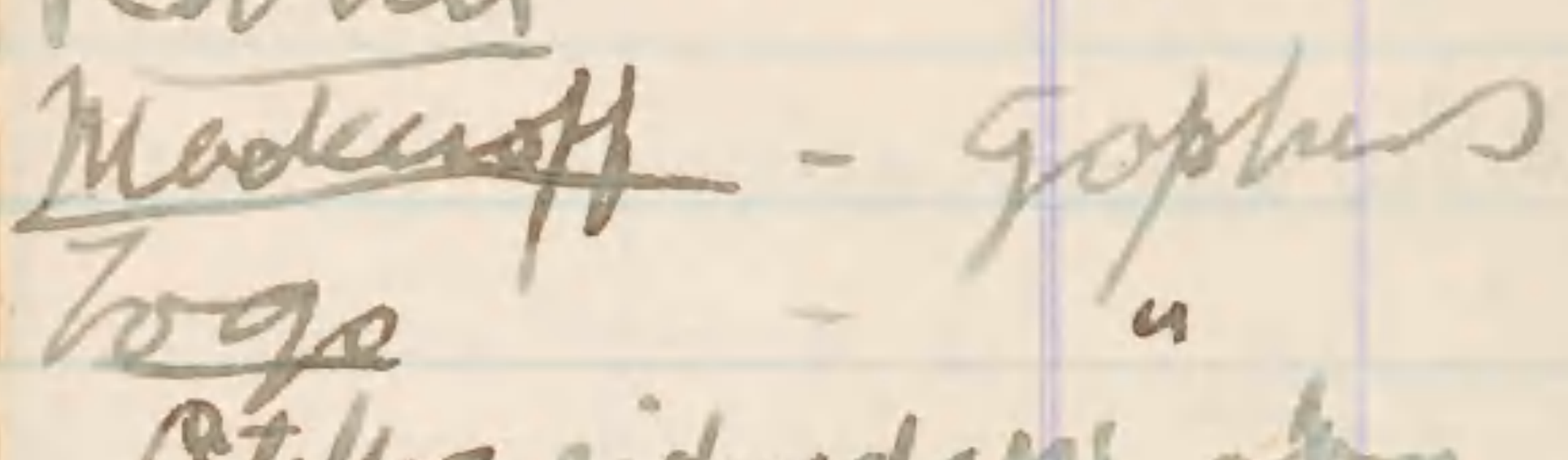

No gopber tills

sthobret

Atrhes riduadapes oftro.

in trulete

sysing glan youg.

Portag Juetion

Populan tremilbicts

Wimiper 9 A.M.

Manily timbar.

Poputies tremulides

Whoms halogmifera

Negundo

7 rastiuns

Queransmiesocanten ap

Solix sured

Viturnom lentago

Coryma

co. Gasainpera

dren

tarix

in aramper

un Piera

- Cosples anarieonoms

en Primses

" Salix

- Eymptroneapas

chagnus

Ehagmus

atm

an

4 
Kamoack 8:20 am.

Necimen 4.t.6.

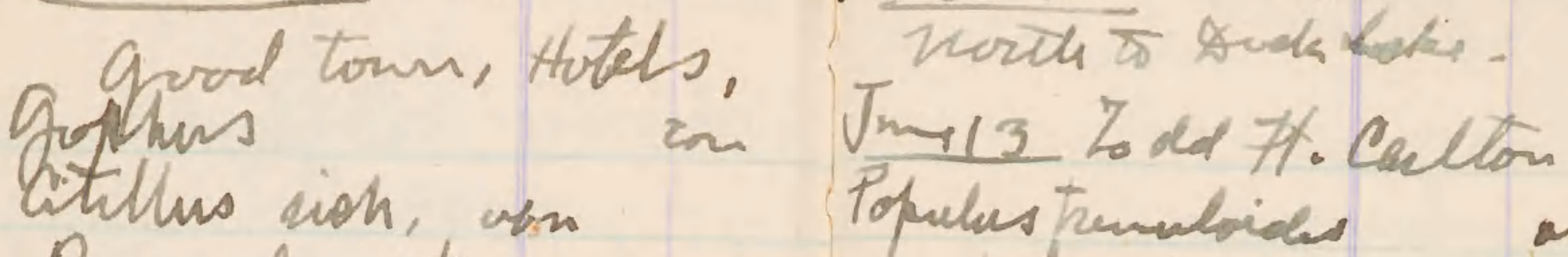

Proser domistiens, en.

Corvus

Populus tremubioles

ab

1. untomifera

Sativ candata

4

Batto esvanosini ofur

salat riga?

4

Conora a quadtom.

thommon hilb

citellus neharbong

Niqundo apus dong tives

Tiny goptinstiels

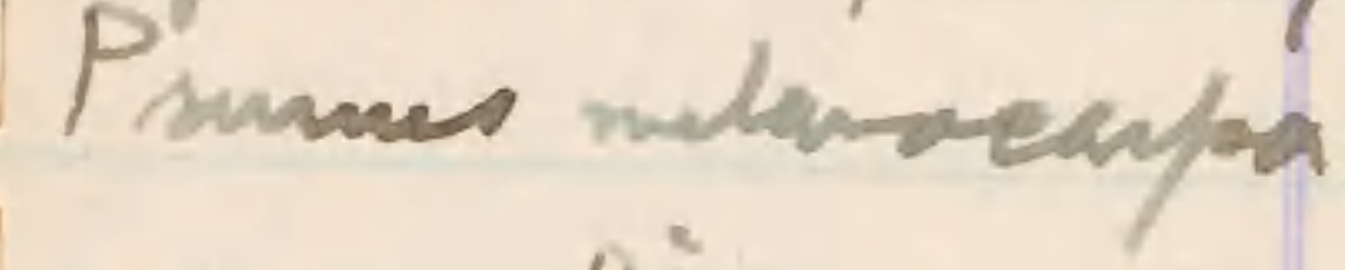

Buchanan

a pins

Amilansties chipolis

Vitarsman afulifohim

durerway gophestides Puhus stroquens

Marefo
Mbelina

Clain

Rosa

goplun hills

symephorcaspos revmasa? "

Qivellate, yophen

Conphes

Eliagunas

an

Hunbelat

Shipherdin

atre

canel

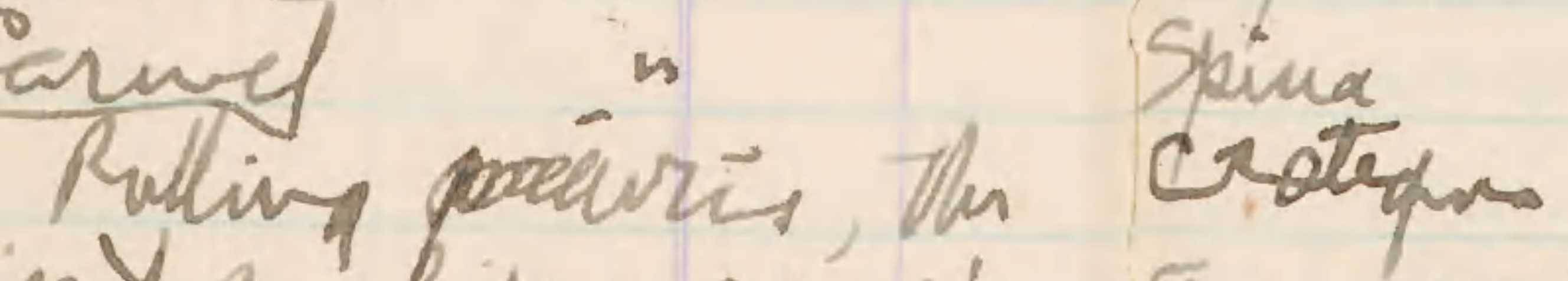

fisy ul pen privi Arosalkie

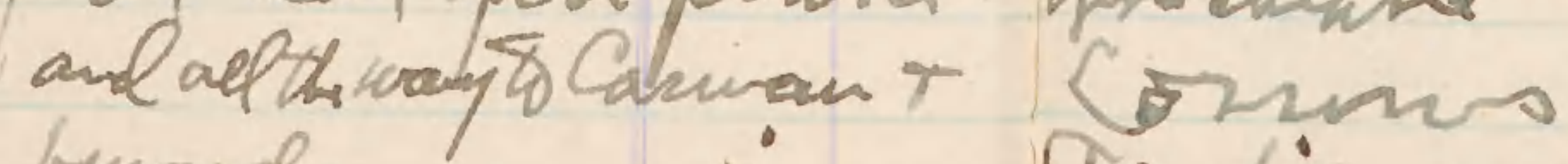

bupond.

Ar lia, asspeñlle,

c.

Pyida aonda 
on prancis

Elagras

syngtorivarpos

gaura

gaillucha

stow Fubea

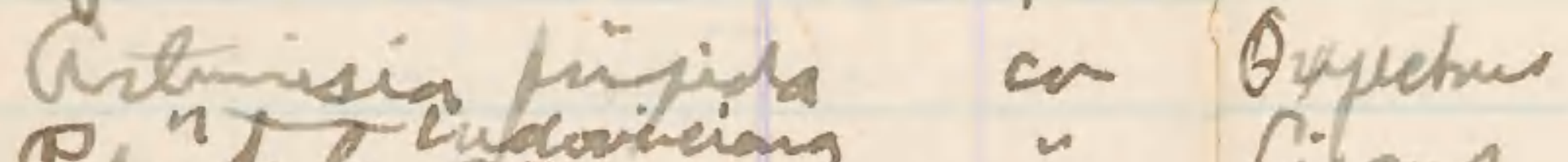

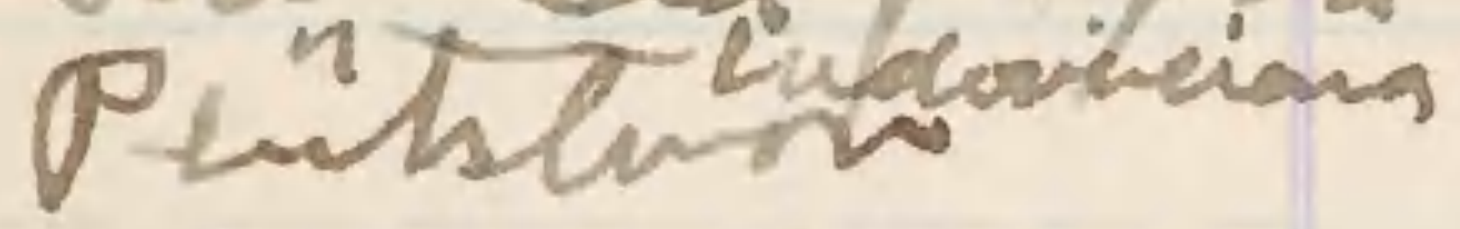

Yhumpis

Poturitha andina

Gyaphatim

Polytak atha?

pesterctata po

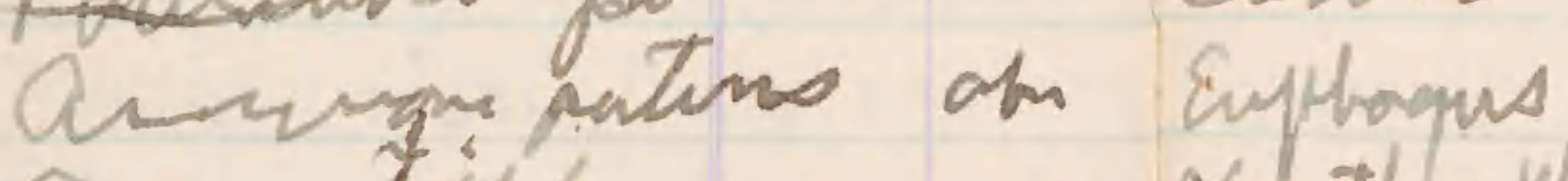

Gerum hiploser

Hraguses

int fush

Iinum posmes

Lithropurms

indotprepto

Comphers comeseann

- Buteo

- Coloptes

- Agulains

- Molotims
Pusk Lated Carlton geis

Hydrehelion

ots Anthyis affinis

¿- Querqudula diveass

w

4

4

- Chordiles

4 Solopspiens

- Empidonox

car xchathraftolus

- Letermsgalbila

- Sturnilla nugenta

- Iyranmes tyramins

Jowins wiquars

Aatrogalioms

Milostiga

Zourtivitia alturamdis

spigullas pallida

procectes

$N$
2

c

3

1

I

con

1

a

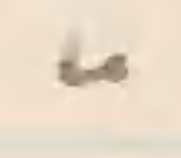

4

$\rightarrow$

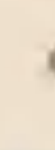

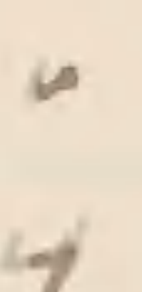

$y$

an.

6

an

noud

reot

and 
Sarde lake $n$. ming

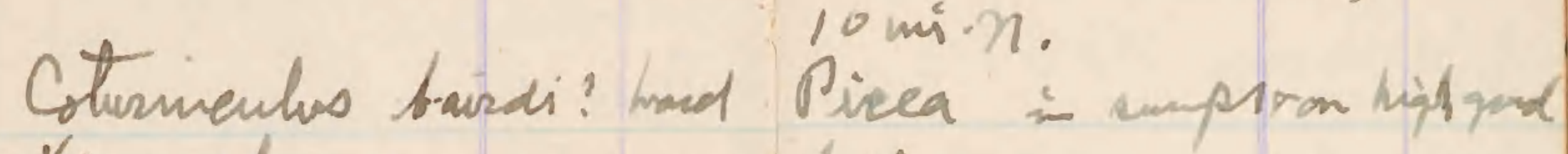

Vins gilow

Gudiovea autias

ifyecienla

Merula mingstoria

Lialia curroverichs

anpibs

- Lasix in manps

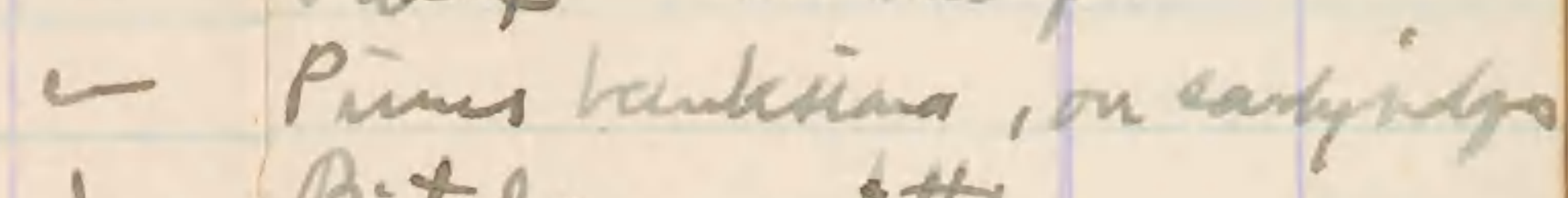

1 Bitula attr

- Popularshombiods alu

c- " baloangfua u

4

4 Juelt Fust.

Pinace Obeet \& Warman

Naman, oupupipinis

Rava

Sinua

e

Soinus hudsemmes

citillus richandoen:

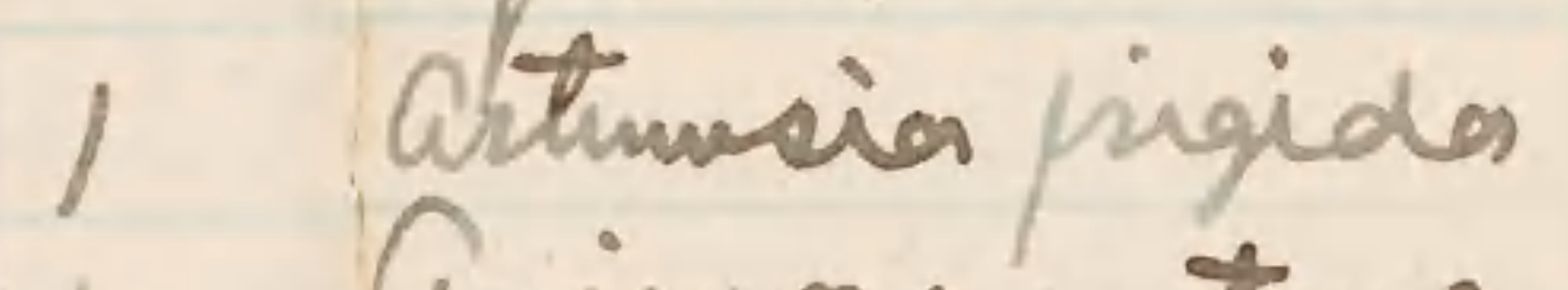

s 1. 3hitu

arm Animone petios as Lipus a means

Zives

- gen tifloms

- Zabidea

" theowopsiomontoumm wers

- Poorabia escaluita 2

Galum

Autenaria

Oxytropis

astergahs

Colitea

Eriqueron atits 
Jue14 Pmieallest ox

Vamom $708 \%$

ro bodm 5-4im.

Pipilo

Wilarfiza

Bordm

Tuliea

porectss

spizelra pallida

Quas

- cosses dpunatiens

outigutula

Cotragalimes

Cmpthojia

- Cumpelus garsulue 27

- Vino gituras

Gativage

cos Lainus

2 Sundriea autira

e

Bartramia

apus Opoothlypar

Tyupanohuos

$\rightarrow$ Eroglotighs

Pedicets

Borassa

can Taxastoma

- Planustims

Butro

2 Aylveidila

Colaptor ansitus

er Sialia cummaloikns

mosts un

Iysammins tyranne

Empidonax

- Zcmaidura

mat, "

(s

Carvus

drn

Xanithoofotoms

Agulains

,

Molothms

m

Exporagus

ors

stimlela

w

Leturns

afo 
Pondua

Bordur.

Drlas

jumpu peoenters, wer.

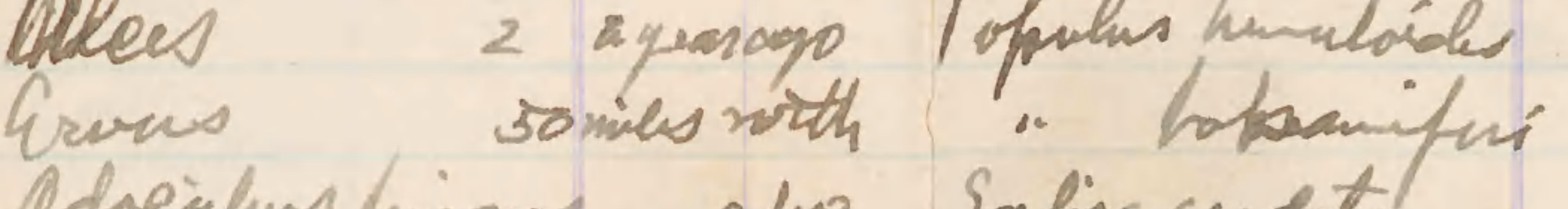

Odocirlus hemionas afeo Sabix cardata foraesanos "D ". many spioen

intitrecora

Secimonshudránew afw

Eitamias voredis cur

Citilus tradalin apos Anctanehies

"1 12linaths con Ribis

siebardens abs. Quesularia

Fibes

Castor

an Rufres striquses

atro Ehagmis.

abe

Thormp tarpoichs com shphutin canadunis

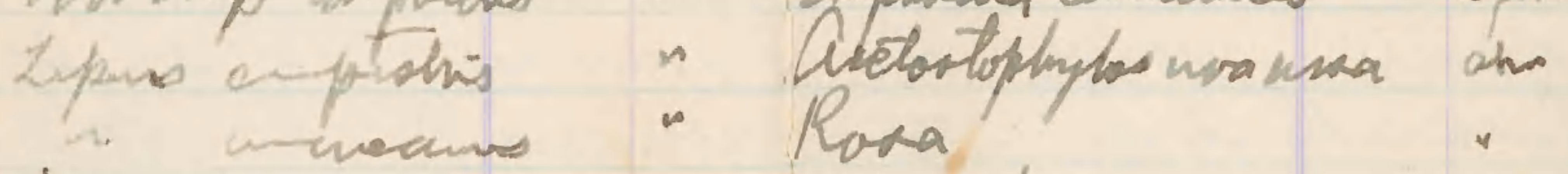

Vilpes pulous

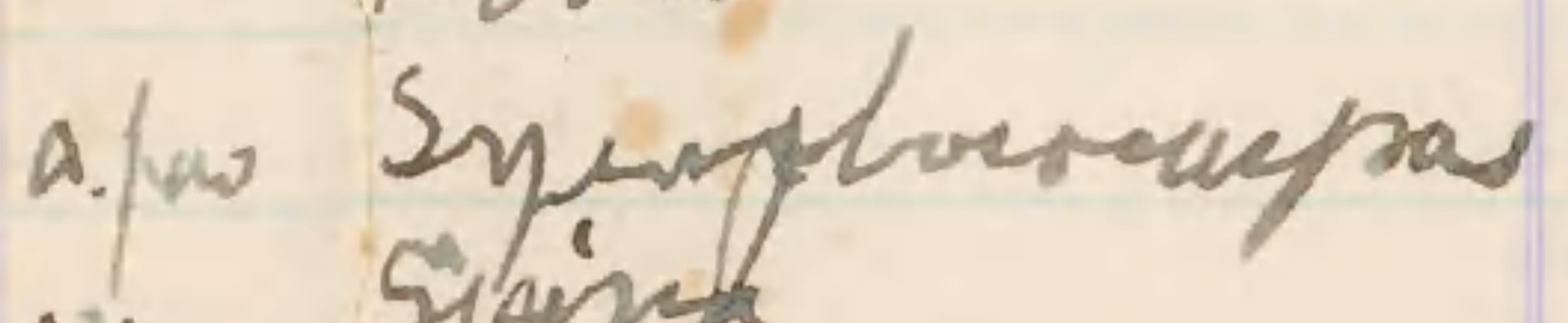

Comis latrans

- syink

com Astinisis vigies

Lymx coskinis

a fis

Putarinos cievegrani 1 Fulsotellapotus

Fatidea

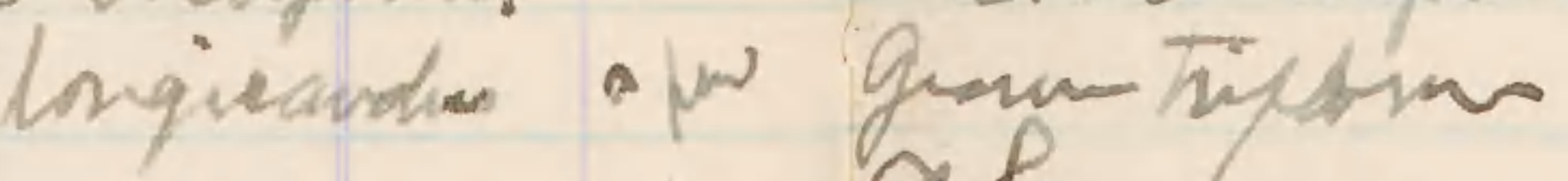

an gecterim

Avengen cundersis

itrenchua

of the projilis 
Vine 14 Left Barden KPly Tuie 17

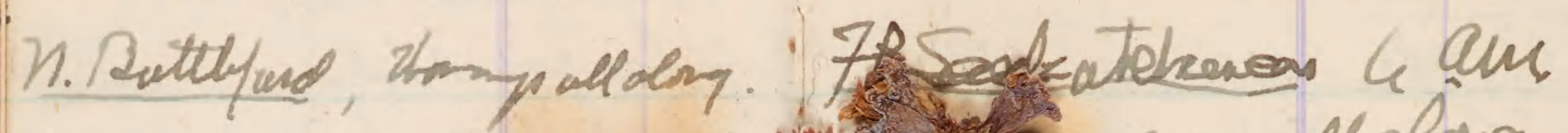

Paysution dach, thanozs sanitype of pen pecuoh dometry. Suls en, all along Wofin aloides an fuel ghin

Elumter.

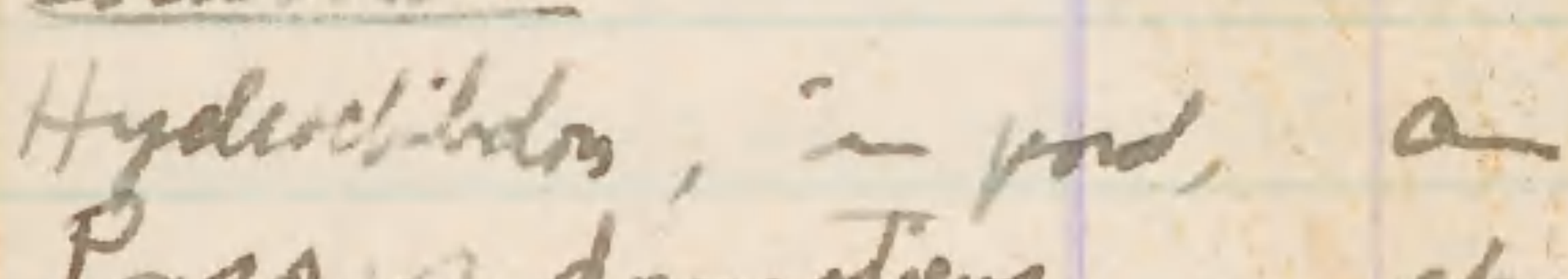
punifua molaveapa Pase 2 donnertiens

spguten polindes

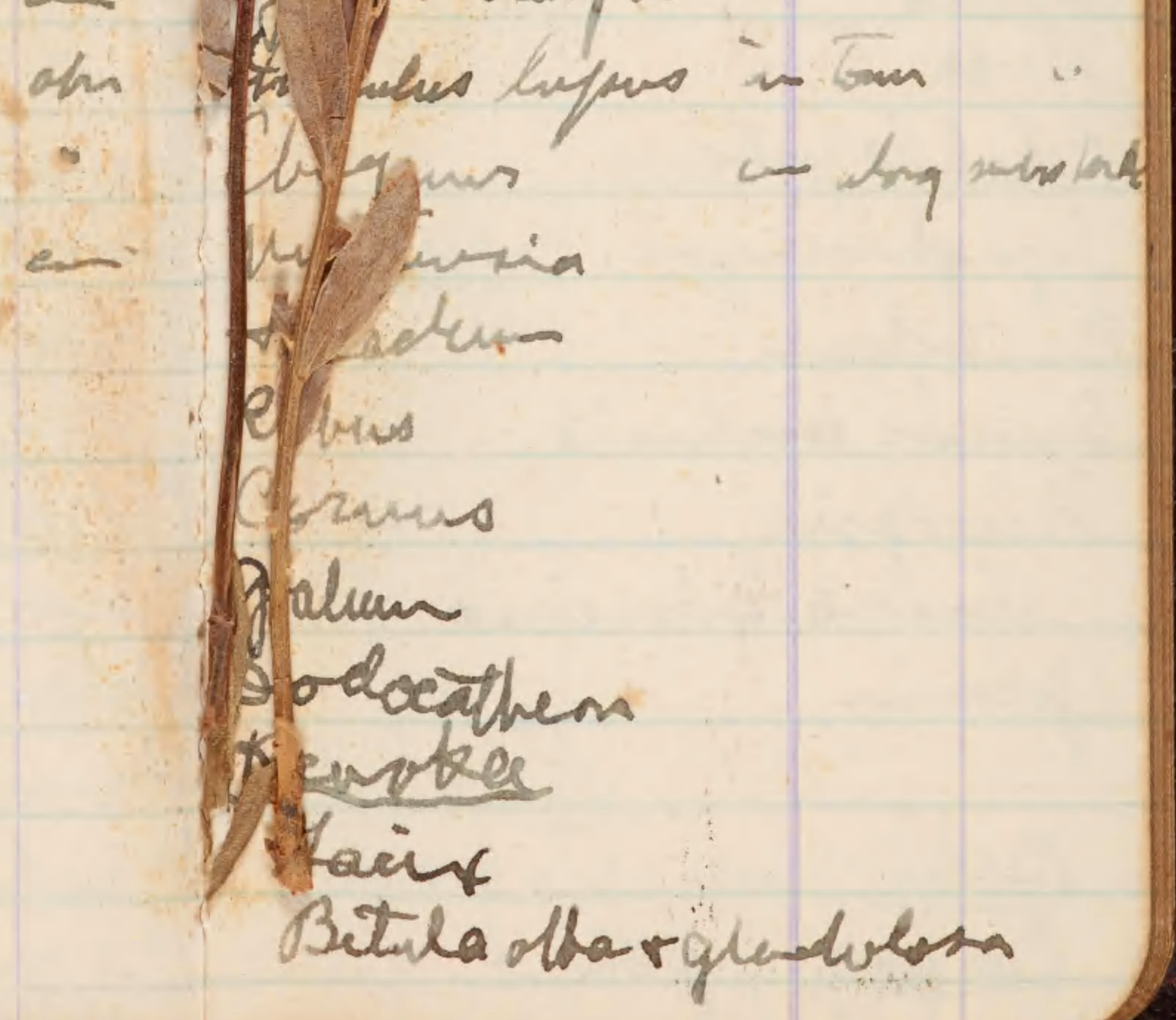




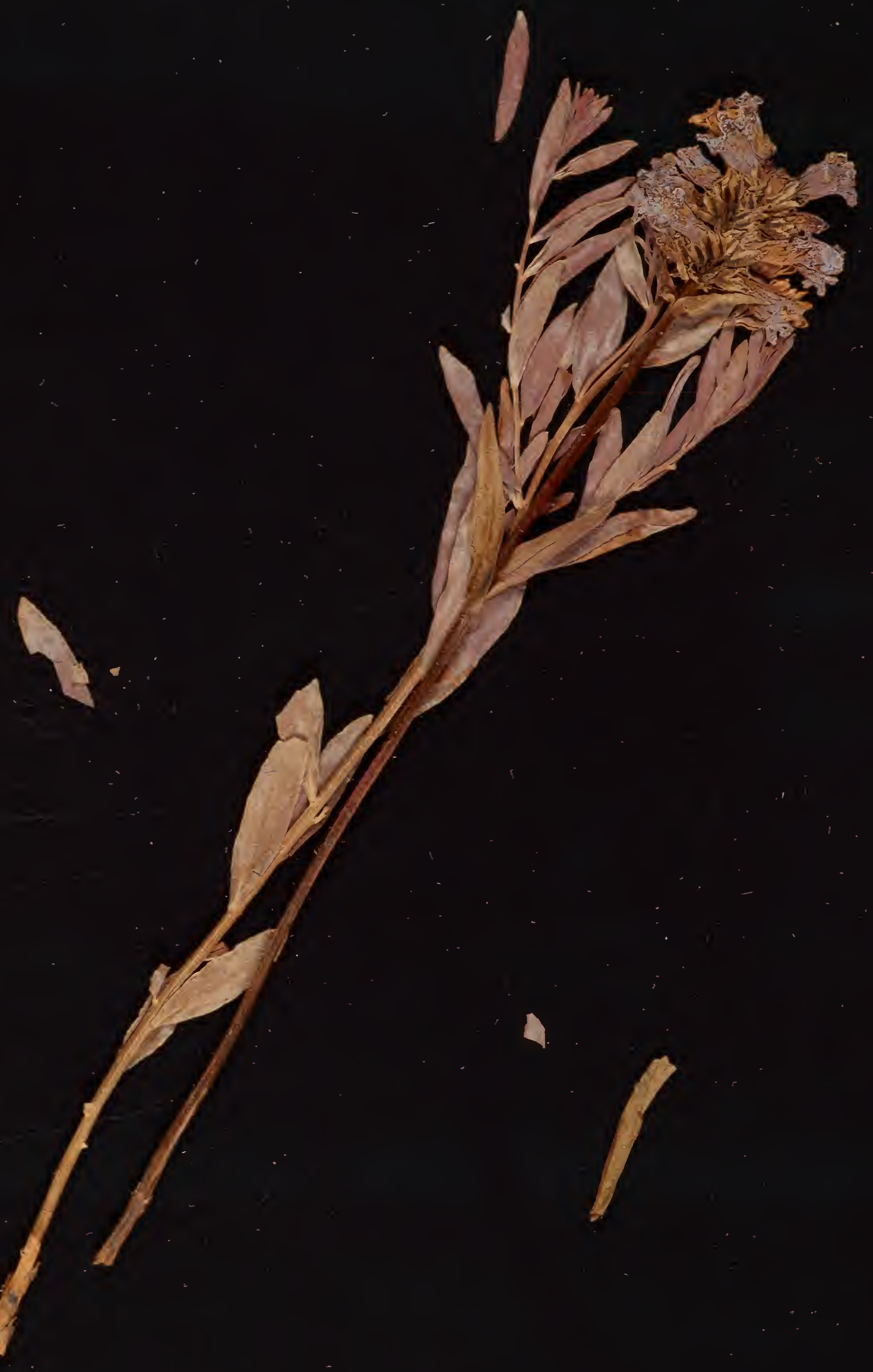


Vine 14 Left Barden kPly Tume 17

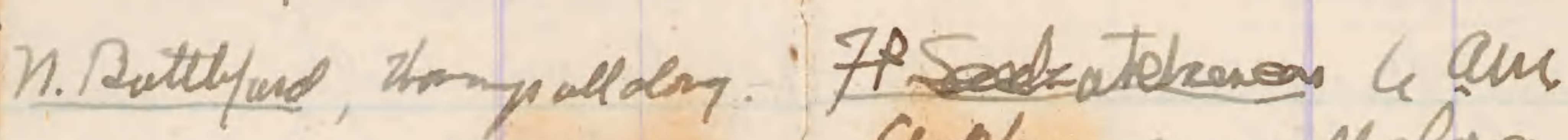
Goplus en. all along

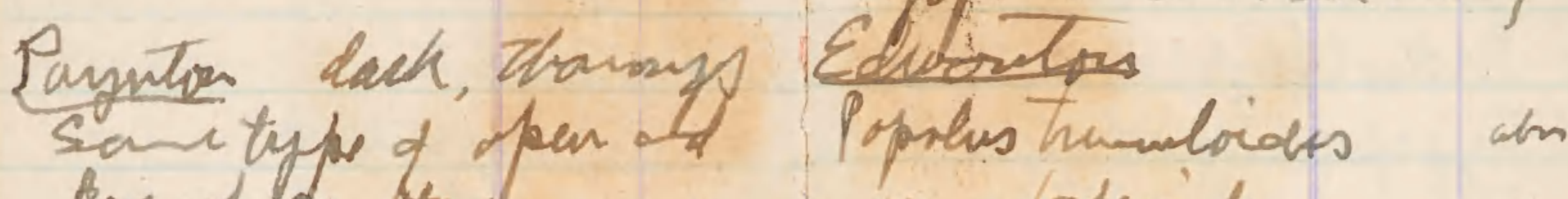
sanitype of pear. Poprlus trumbiaks an

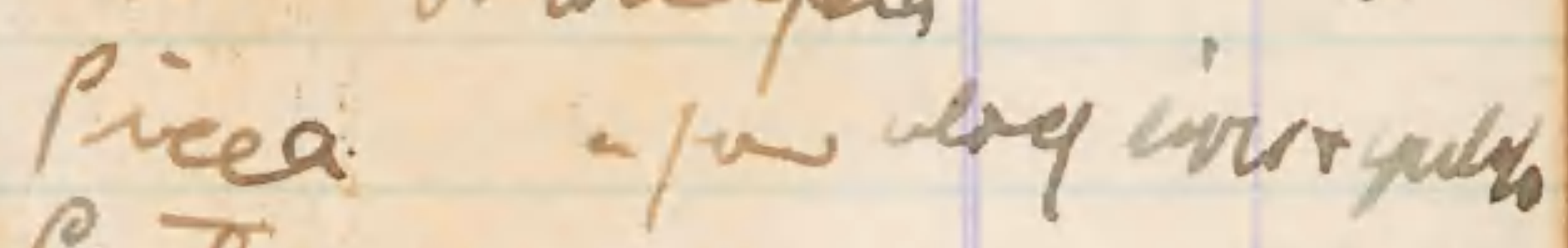
Cratiqus

Nequede incrans, don.

- Solix cardota a. mony 20 . Sindanchin Prumua noloworespa

Edumto. Rosa

Ayderobiblon, in pad, a isymploweapes

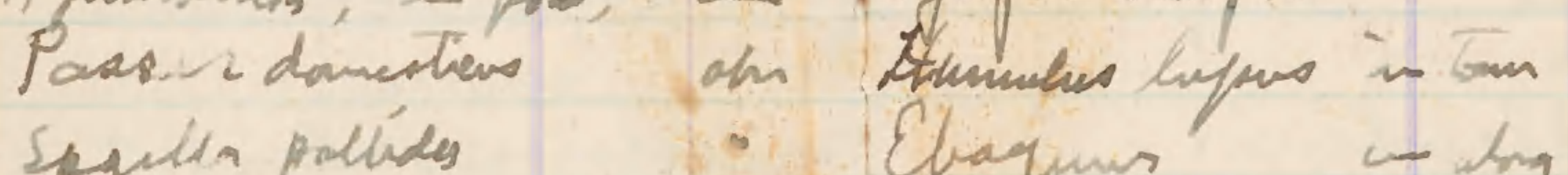

spgiten polides

Ebagums co hig somont

an Wyrtumia Higuadur

Rupios

Cormus

Galum

sodoezthem

Krenotes

Laint

Bitula oblo rgladulora 


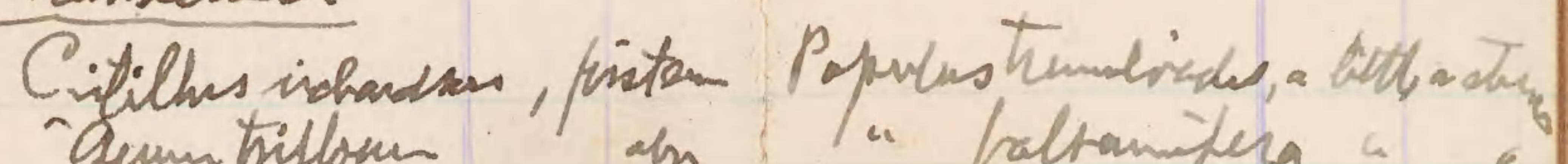

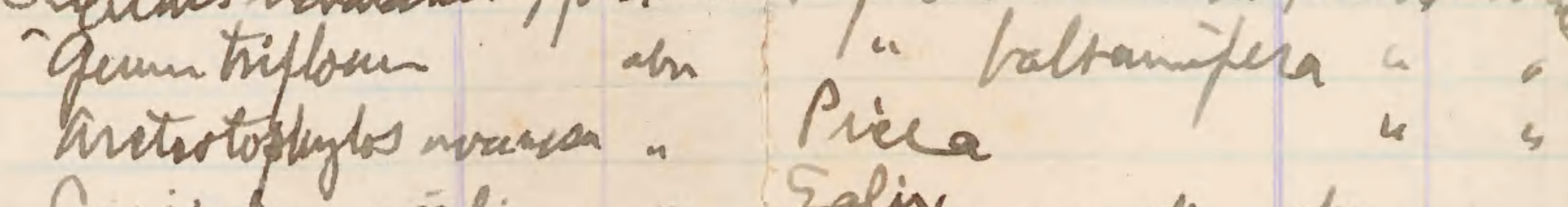

Time 18 Bolopery, 85:9m

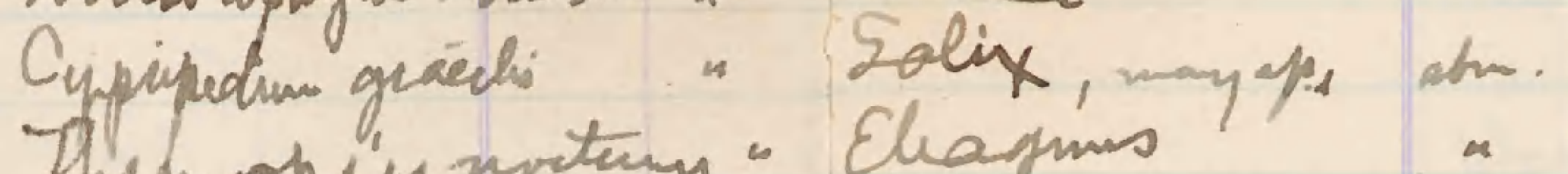

Dhinopies unteins Eliagmas

Real been

Symplaneapsos "

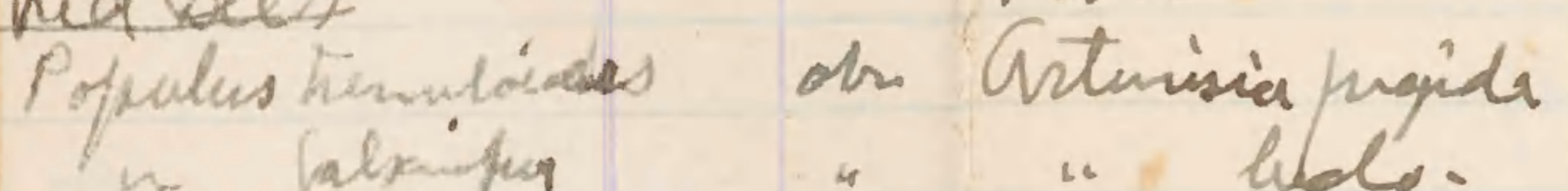

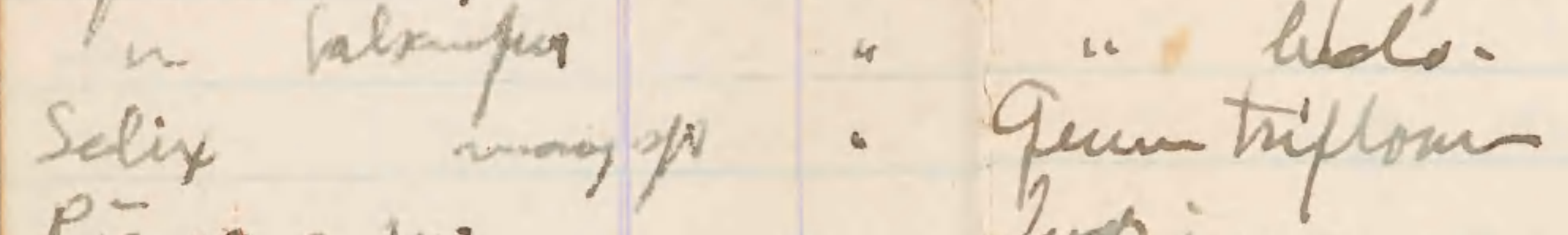

piéa, a jui

Cempros

Aproqupua ovadersion

spiva

zopims

Gynain

Patutilla querion

feranium

Pitentilla priticasio

Symphomeanpas

Qterioks

Dumstiplonn.

Cinumore onothás

theropsis

citrming hadriveinso

ripp

Geramin

Boden mon open prouns

Lthbridgs

Calpina, plaine 
Lthbiidgs

with

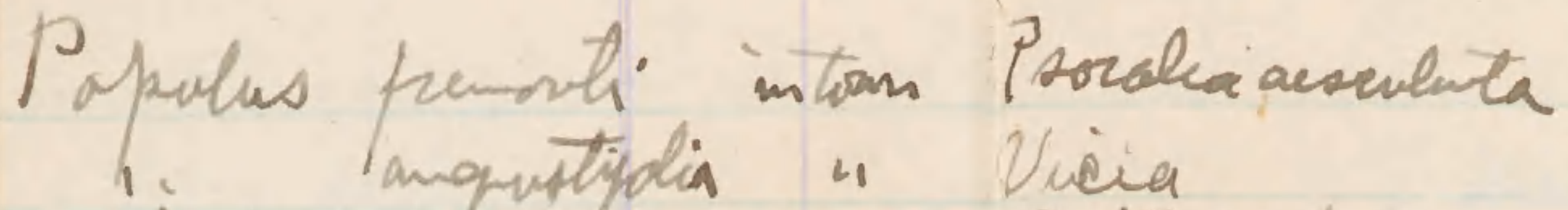

Salix

Frahum

Astraqalus

Migundo

intous

41

2hinus

4

Eleagmes

Rospla

Mus hilotata ope.

Artmisia ema .

n fiquda

Qmentwehies

u undooveras sp.

Qenutia nimsonumis

sp.

a

4

G.

Gaillaclia pulphulas.

Chove intuppolias evos. Iny PA

Sinas

yollee,

$n$

Zaqadins

Anturaria

Cochilia

Conguamila:

Zinin persums

Doutitina der

agiatertin

Anensme

mut losh

Gunira

Pentraturer

Mabristan oreinem

Erriogoum 
Time 19 Lethaidy, 9:40 ams.

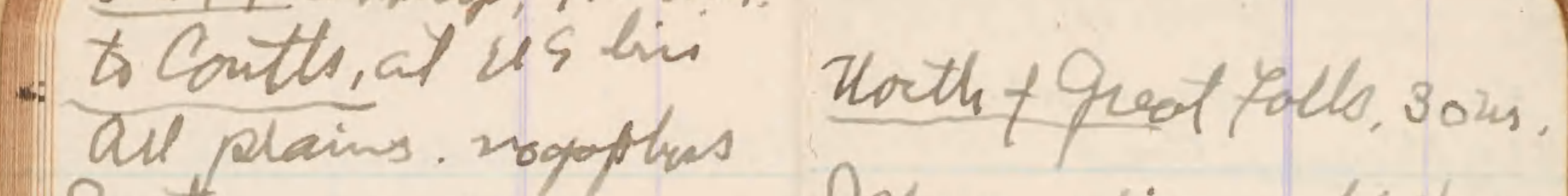

Couts + anth Colomorpisa, firstsen

Achinera pingeda

un pours

in Vaughn

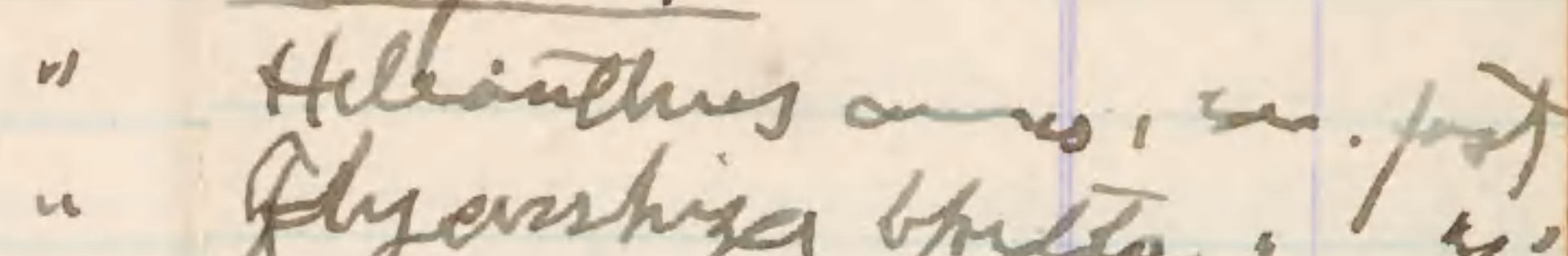

Symphoreagors

Sorodistus

atriflex mutallis

Qpentia

1) piimins $\approx$ pot

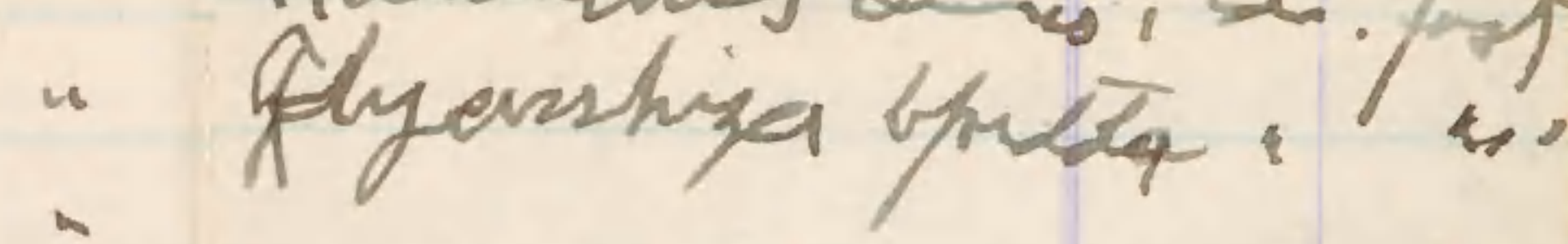

" Sumpion

" no qaption teturen

- Courad * quet Folk

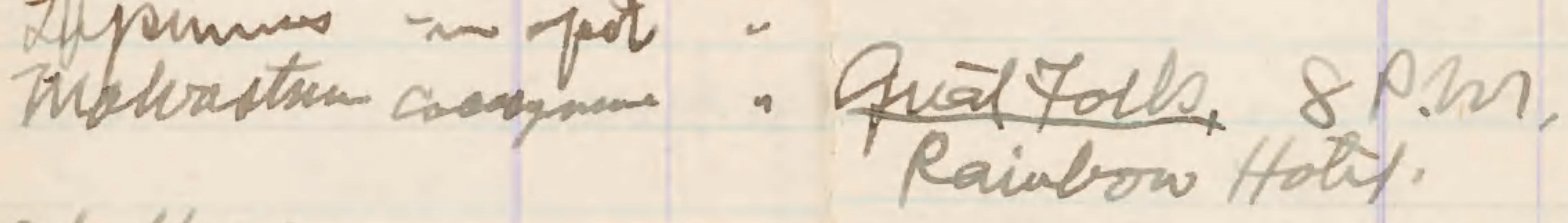

slulbype.

Zenaidura 2, kint arm.

L 7 an

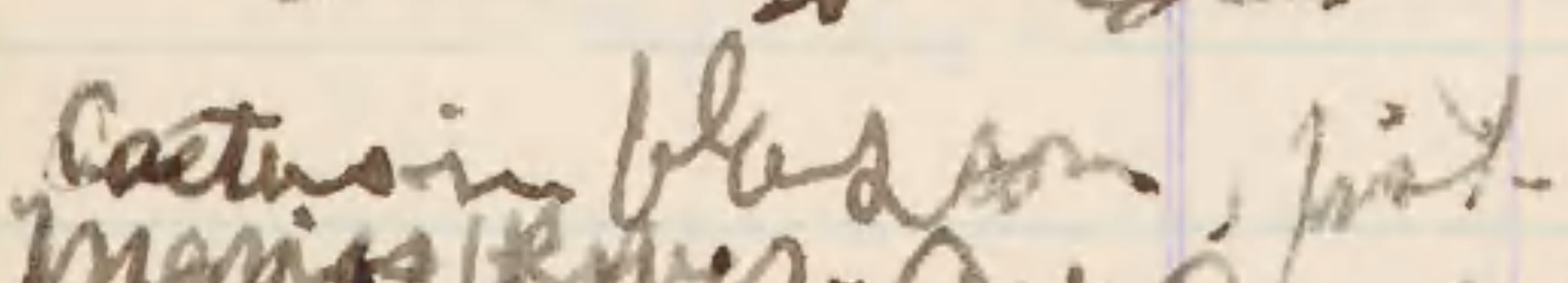

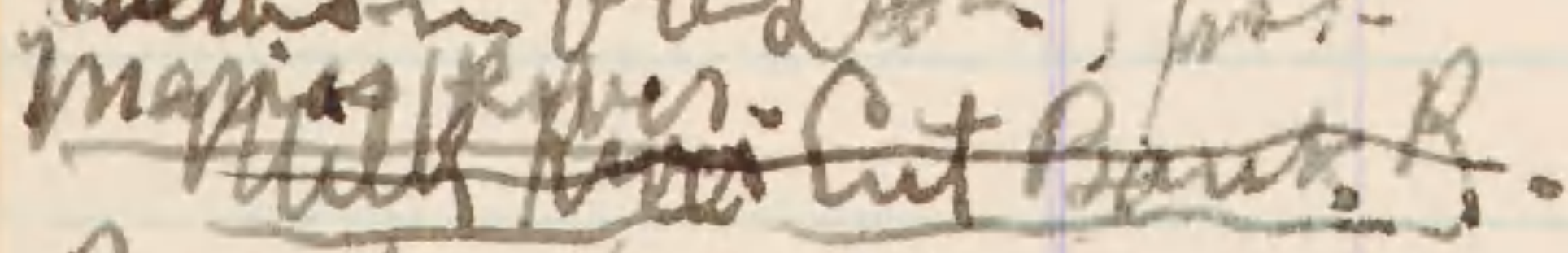

Poponlis poumti. atrs

.. avenotiplints

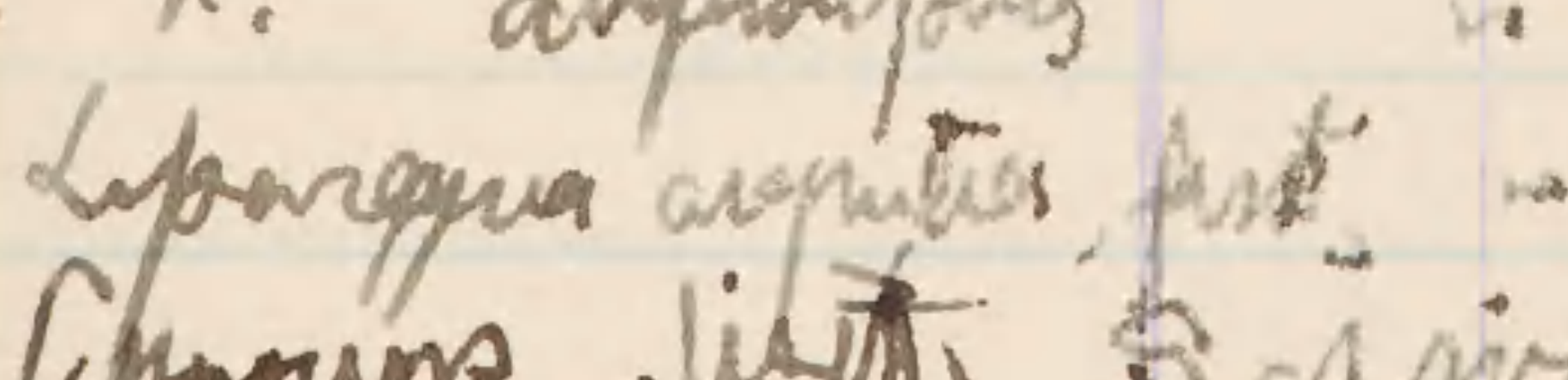

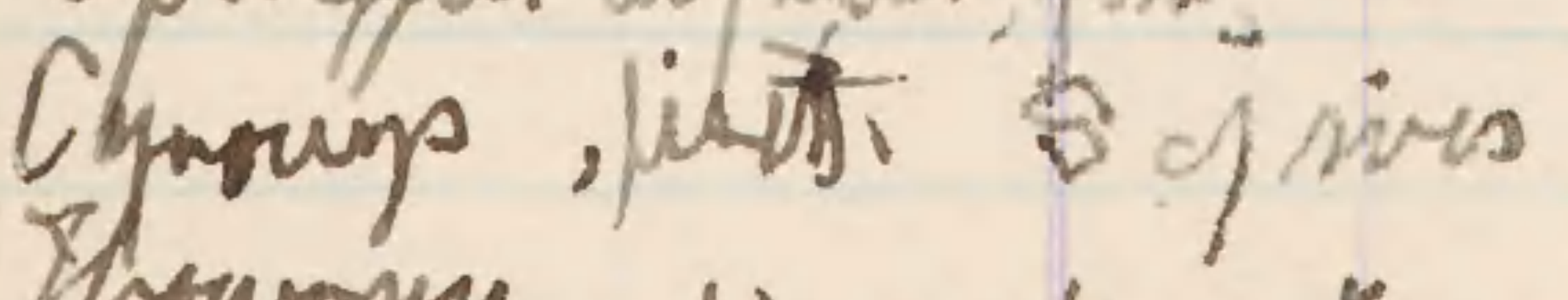

Muaphis "

Runber data a par 


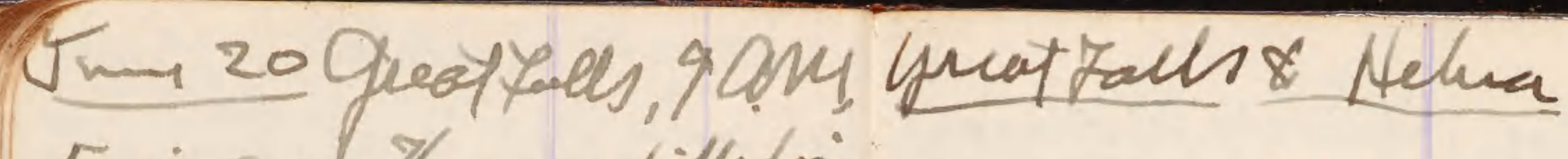

* 5 mi. S. w. Thampshills tepia Curlw

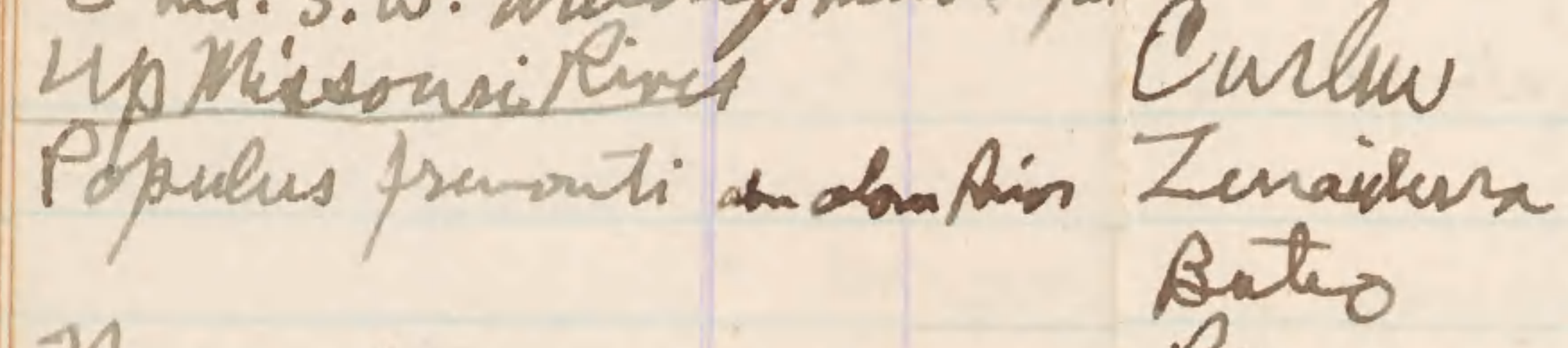

Negundo

a Piea

1

Salix opi on taloo phaclewa Rhwotildista s.alpes." Corves menewar 3

Rosa 2 "Colptes geyecrligs

yueea in Marm

punitia

Oitumisía cana a priqids 1 lindo. n' dradurembitio

Gaillardra Cinens $\infty$

stapdinis

- Cangor

- Craig.

- Carda

- Wreferak

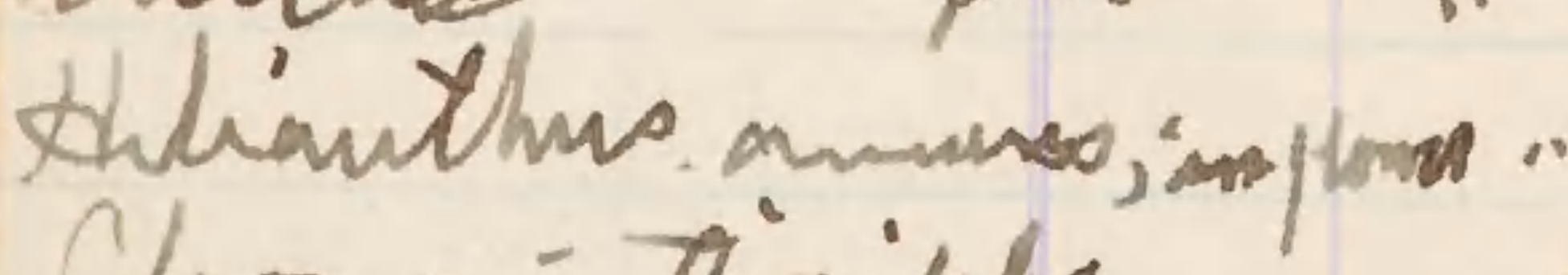

Chome intigiples

Wulalatus alva dagiR.

Lupinus

Whloration recumese

Gouna cxceinem

acodefinos spuacians a per

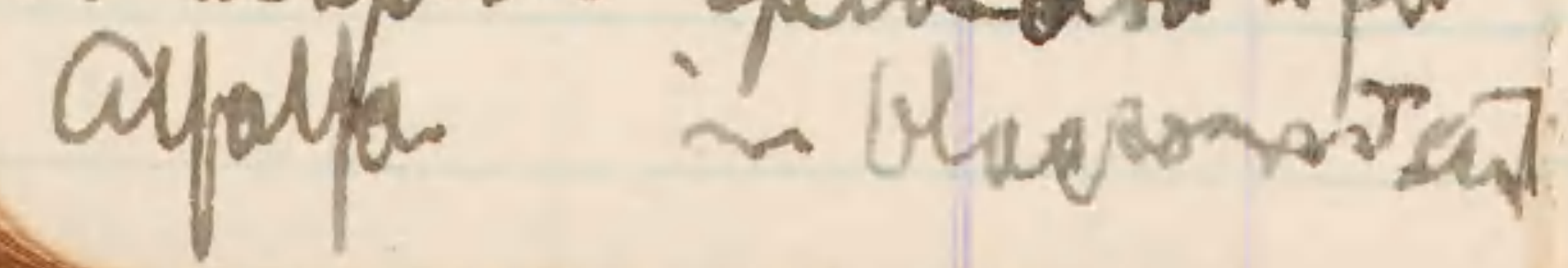


Hardy, boging 1 Campon upablf Cuese

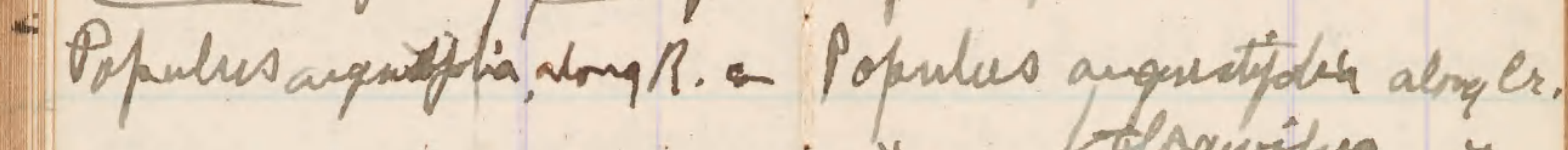

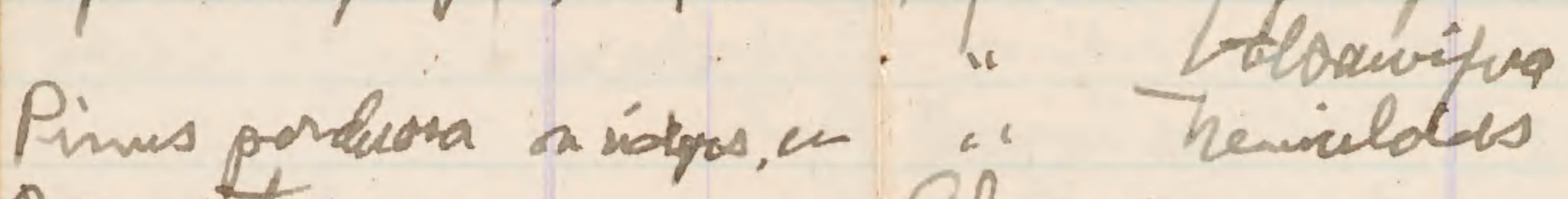

Psendatanga

- Alums

Pims Mritio .alitly Bilula putinadis

Vimifuns seopuetom ". Salix

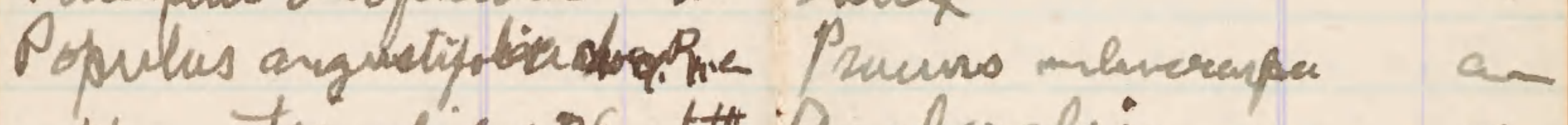

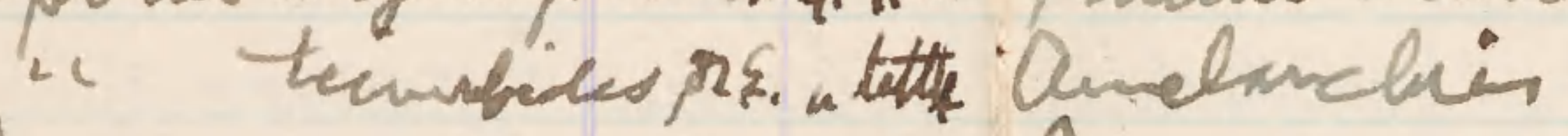

Prunus melarreapa is Pues po bova on indas

Crotrepus

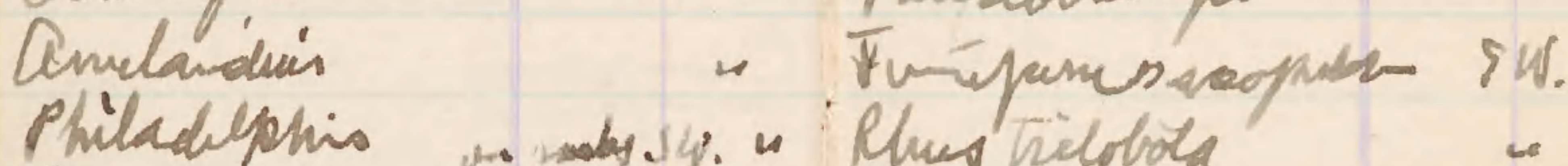

- Pansotruga

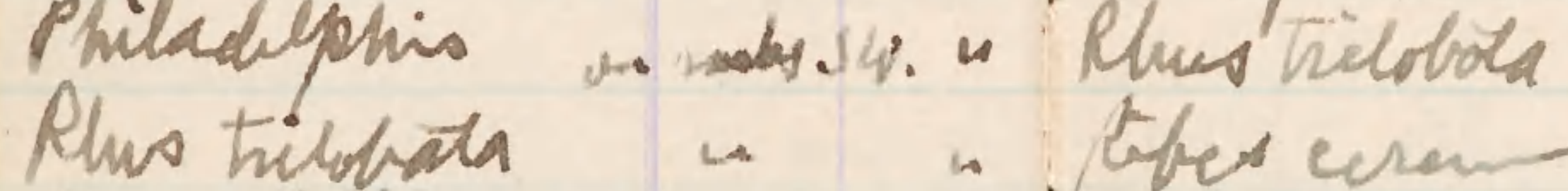

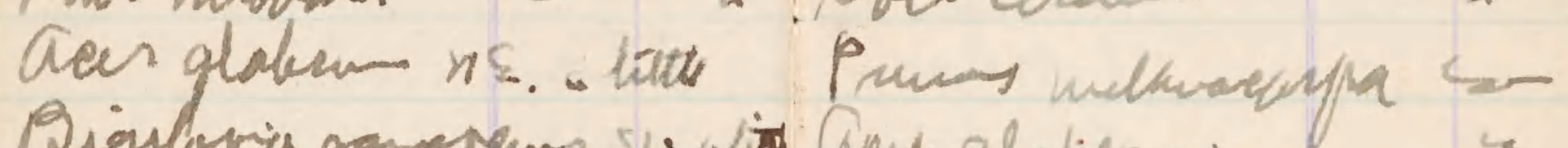

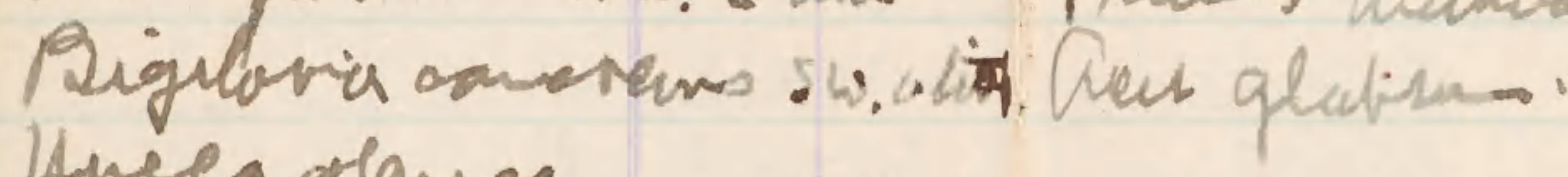

threea glanea

Qpuntoa

arsopiras ypeion

Roua log

Whatlean, arn.

$\therefore \quad$ Out ite Helua Valby

Arturisia riduitota.

arid Transition

Helua 12 g 9 Pus.

Craig-endy savyon 
Tur 21

Jme22 Buth, daybighl.

- Livingston of Gardines. Beer todge.

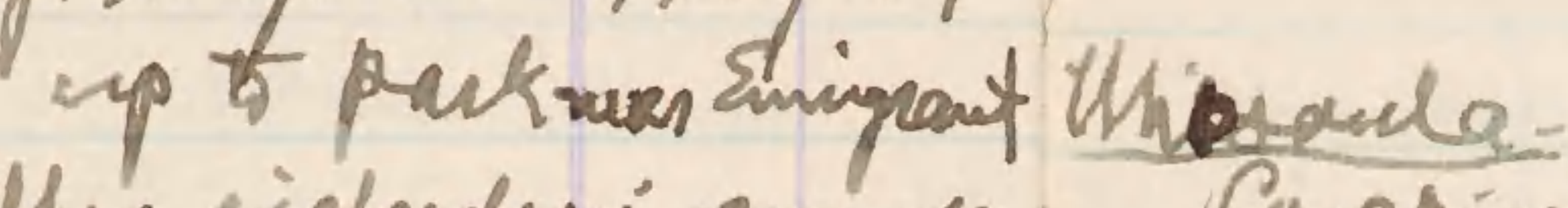

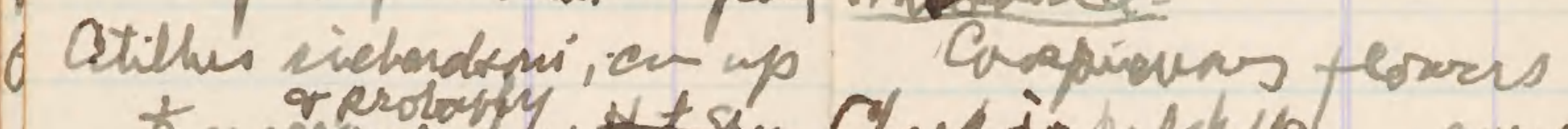

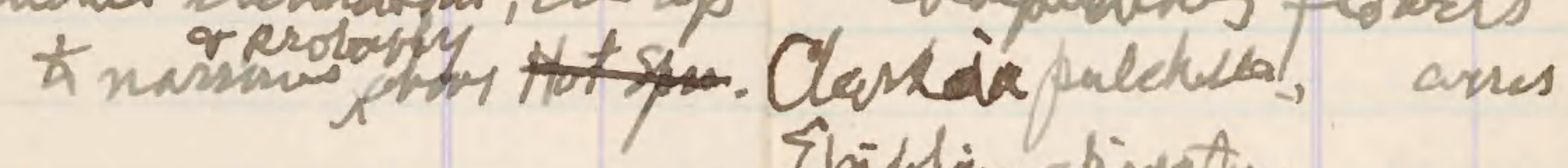
Eloibtim afivertim

atillo armatis, 1 at station Phacela

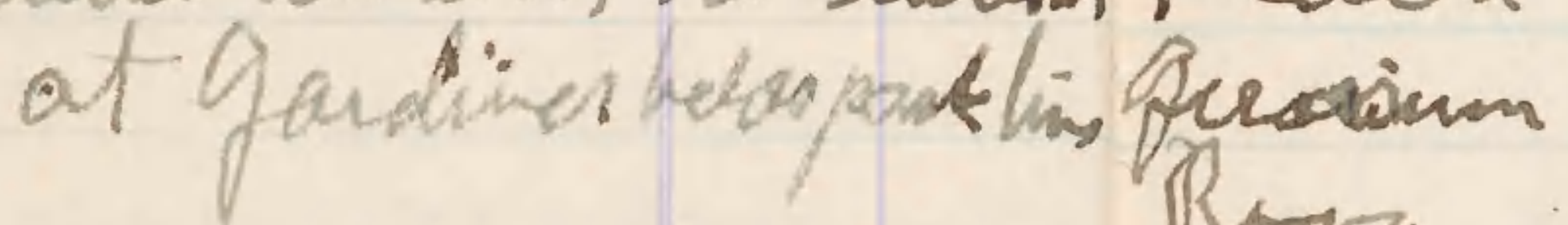

Roda

Espine

Pretstoum

Gaillarlis

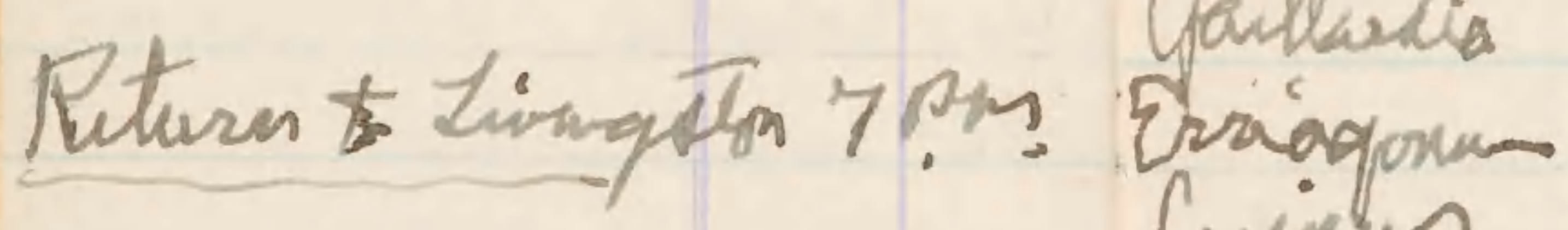

Cunevs

achilen.

Conuasia

5 encio

Custallija

Grealivin

Ricios incterendox

Ravilla

- Spokane 4\$8.30 Pu. 
Tme 23, Umatilla 6an

- Artmicintidentats an Qrygopsis Chrypothame tostiption?"

ah.

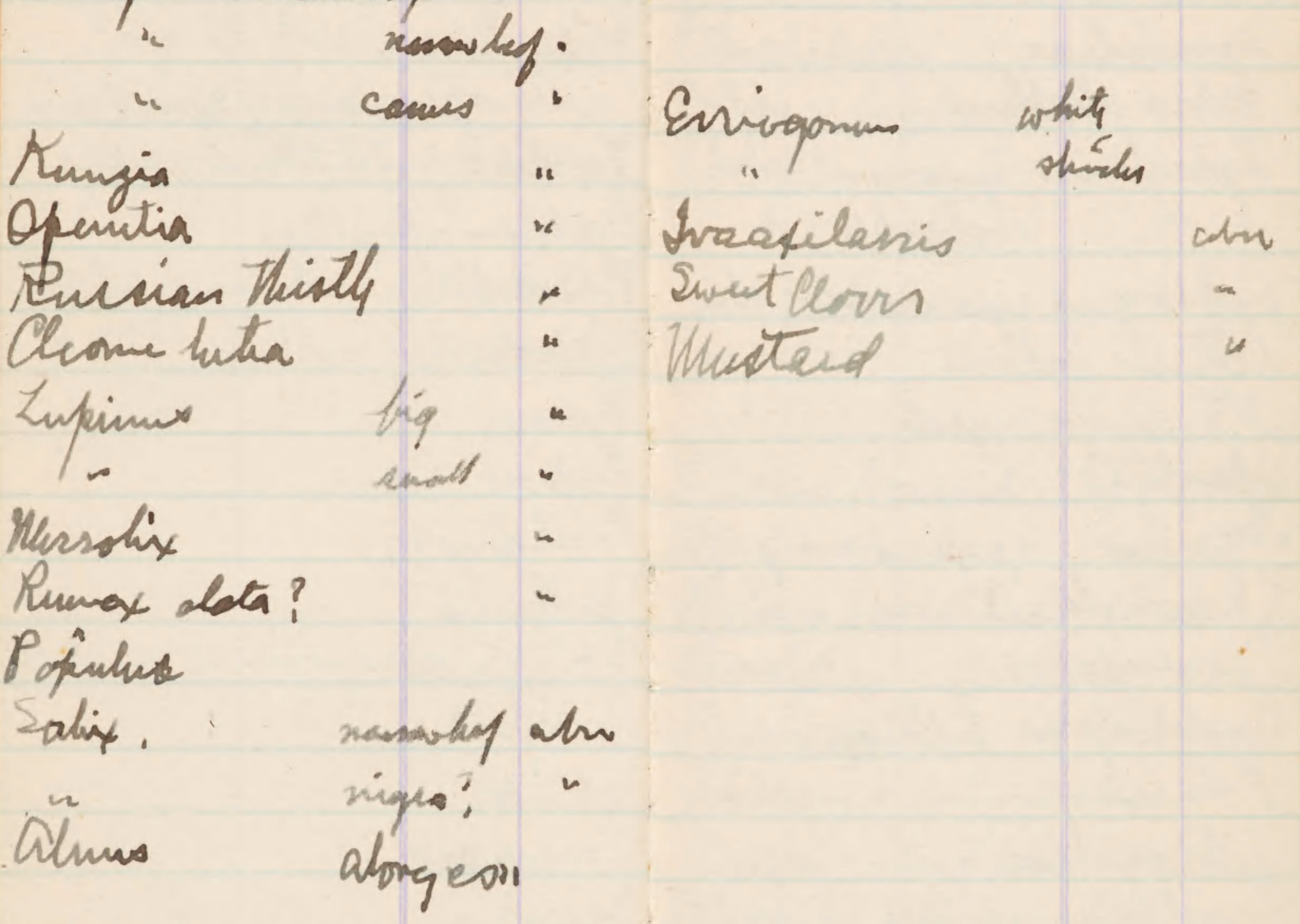

trinitglia

Plantogo potagines

aho

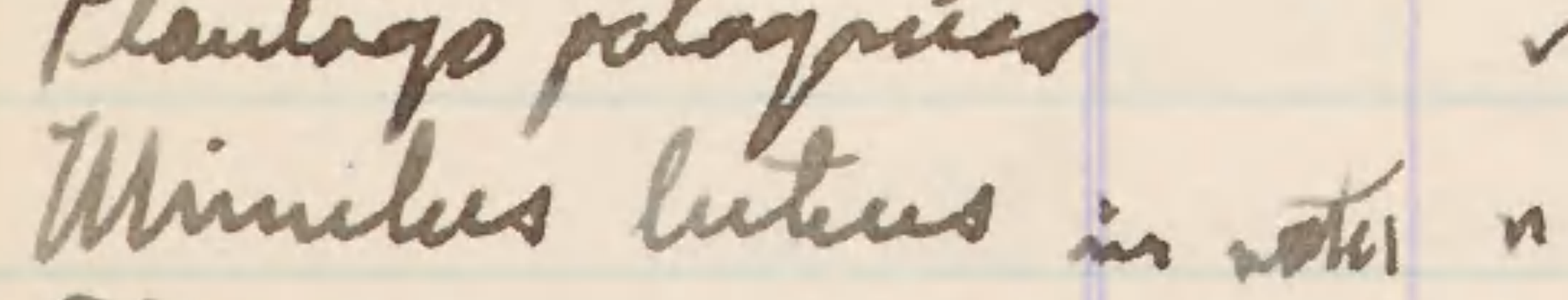

Abronia whis n

Pharchis resposias -

Heliatiopen

Elodiim

Euptorlia couputisa

$\infty$

$\checkmark$

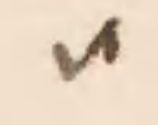


Unatille gn 23

- Mnjetarecrax

Oyyches

Cotitis

Znaridura

Fales phadena

Iyranum typanas mistiechs I

Euphagus cyaroeptolos stm,

detrum inllodi

Sturnulla nuplata

speotyto

Llains tyg. fanily

Clavíeda?

Carpodaces

Compliopiga belli yg. co

Astragalius

Denchoie asstiva

Plamentiaso

Covors amenearmos

Agelaiñs

Melarpiga

stilgadeptiug ?

Riea

Curdeils

Chordecto
2 Conves simath 2 .

2 Dlyierelus cincrasoms I

en Pirochulidon

Leteria v. lougieanda

Colaptroceps

hend

I

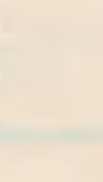


Ramela 2ur 24 ,

- Pins punbara apuors. Baloamarshiza sag. S. con woutiede a pro dupinus yuliow we marsyana, dro. the Pieca Peindotonga apis Lurix acichntals Salix Whonas uning op Populus trichreayso, How

co thenrpicis

- Trajua

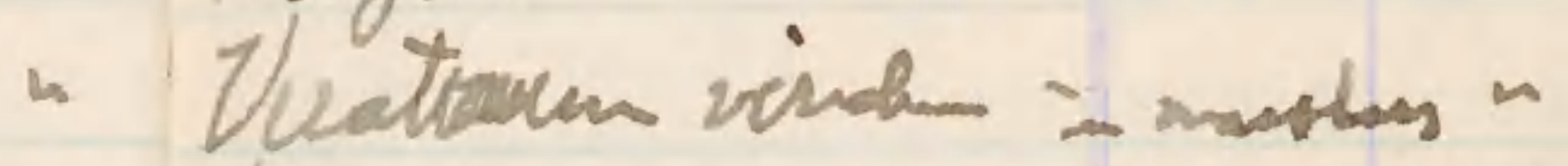

- Limeas ob

"Clintosia mifloss Loniara uthrouns?

L

Uynilegin Pyuda

Gers glotron Cinassus

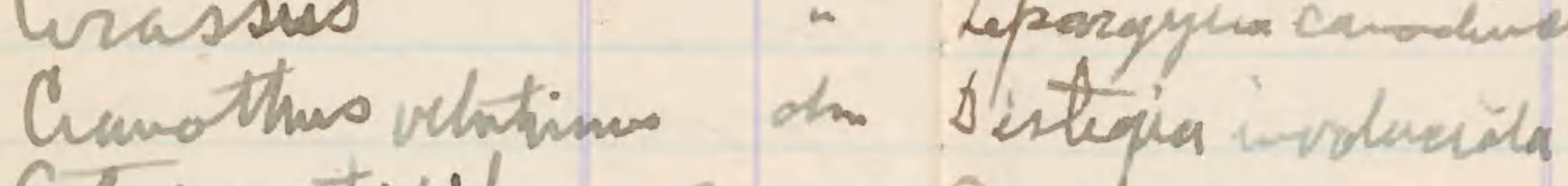

Arhmisiatifida S. $\rightarrow$ Syuphomoves

Piors Holodiseas

spive

Desfecis ipenos

Paduptera

Anctosteplestos wa was

Soctus

Vaceinem ovolepich

S、 $\rightarrow$ Quaclanchier

- Aratulivia

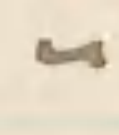

a

2

$n$ - Ptrichios

- Canaivo

- Brodia

- Callichor tis

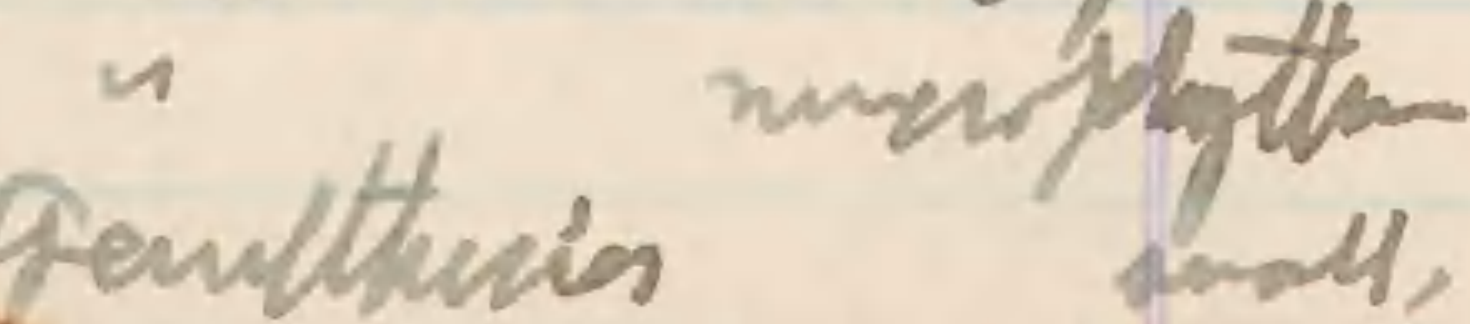

13 
tavida. Miru

Yoboter lity

- Coefbloens Pidens

Anplots

colaptes

Eefidmax

Pipils

Pivanga lnds.

sitta mesoni.

Spigilla arigona

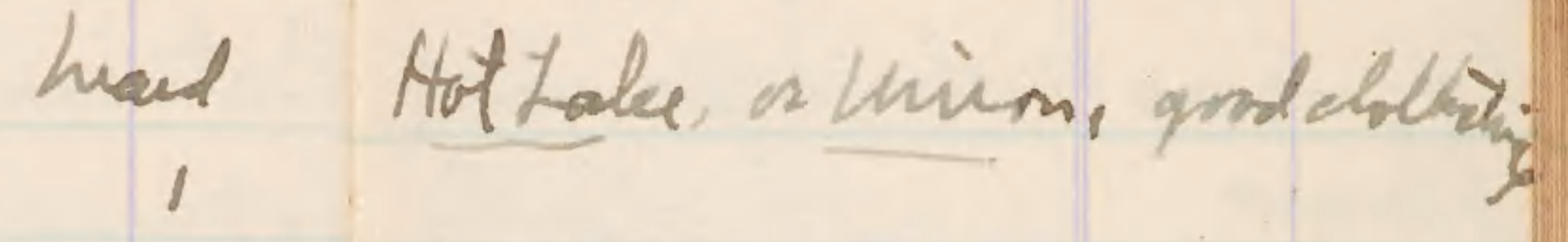

hand Ilocasel - toppidge

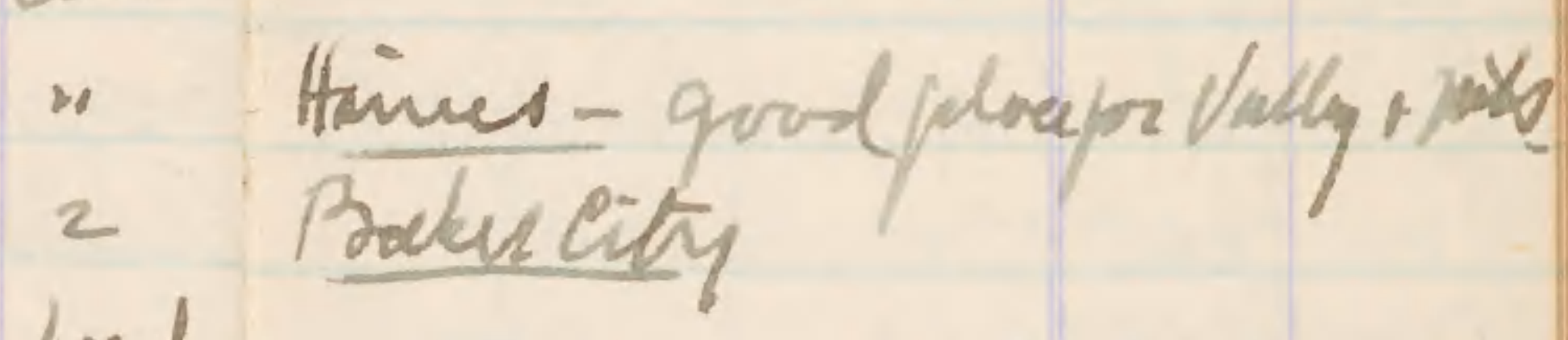

had

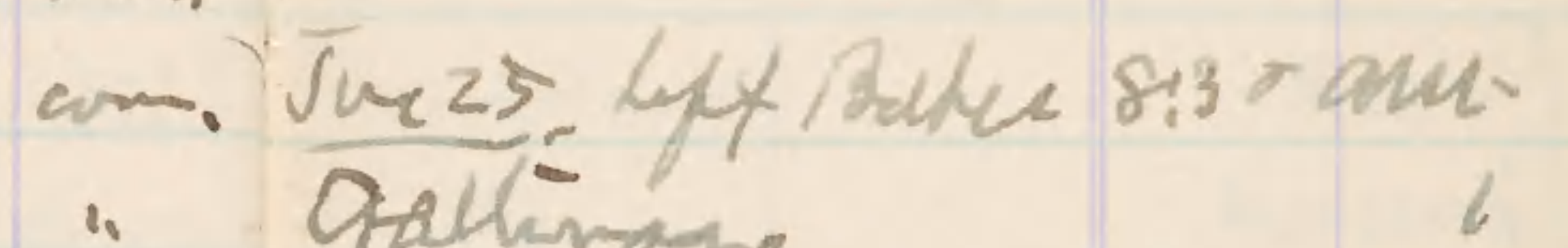

ino. Gathinage

Prives

Planistion

hand. Expthagens

1 Cignlains

Hylocidila

c Sturnalla

had Miloepoiza

Calourtio

Gewors ispted

Seimus

Ettamias

Callapponnphos

2

Atthes edunhians atm.

Thoumps

ans:

Comis, many with Ropes 
Babulity

- Pipulars tridveays shog T. Marnota

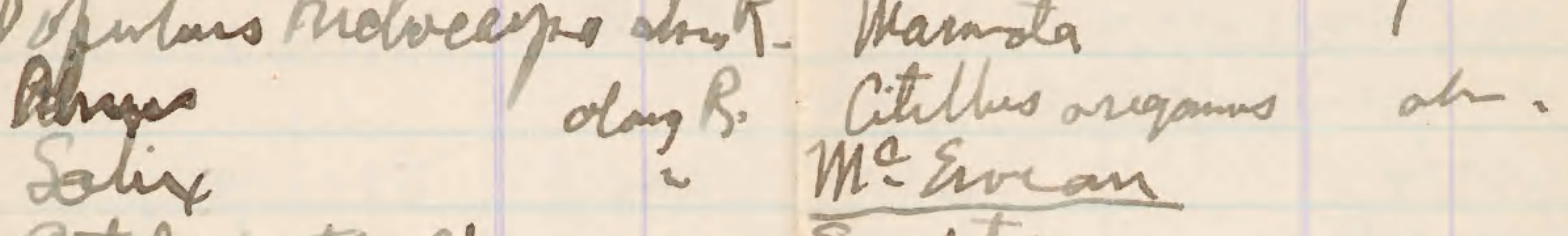

Pitule fontinalis

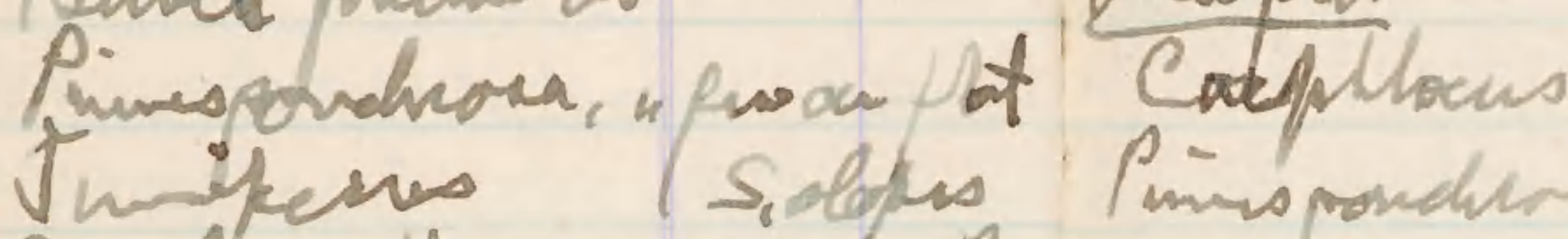

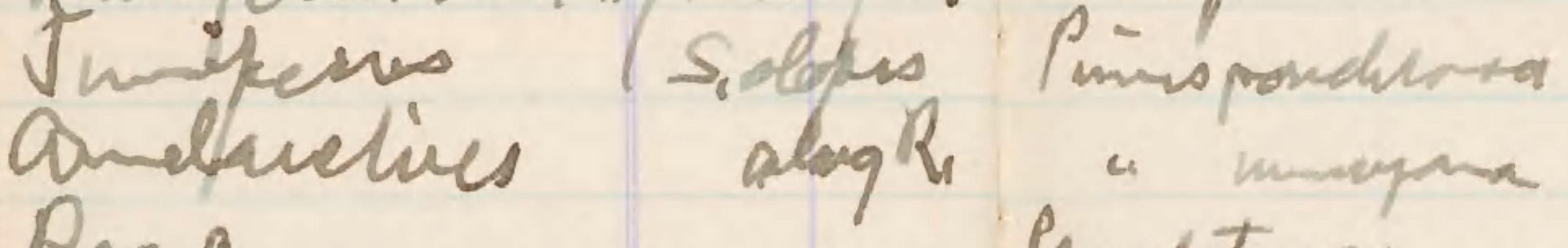

Prae

Conuis

coteque

clankia

Ialusbry.

Cilus auran

u

Kungia

Qniturisia

cheyrothonom

Thourparus

Pinis pondioma Pandationg Lacix

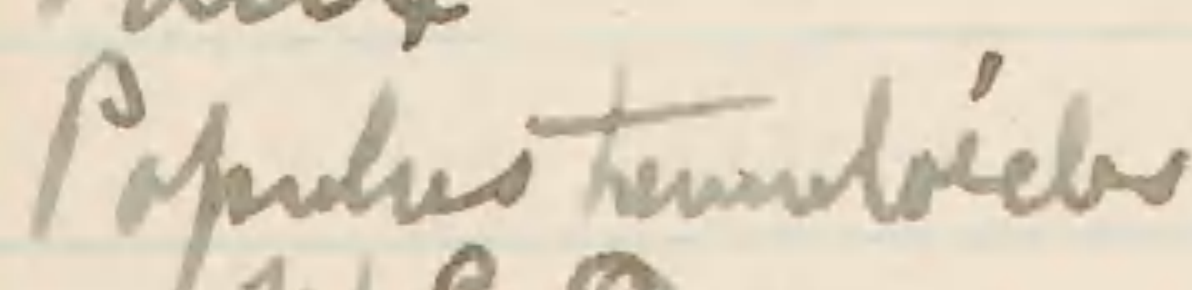

Ule Even

Pinnomimana Pieea
- Prudotura

a Jaria

n Apies

Picua

Ceavattuns weltimo

Smind of indy 6094 al

imanpipion it yellow finc forsit

Jonstertey

vivipul oceiditatis

Whitury

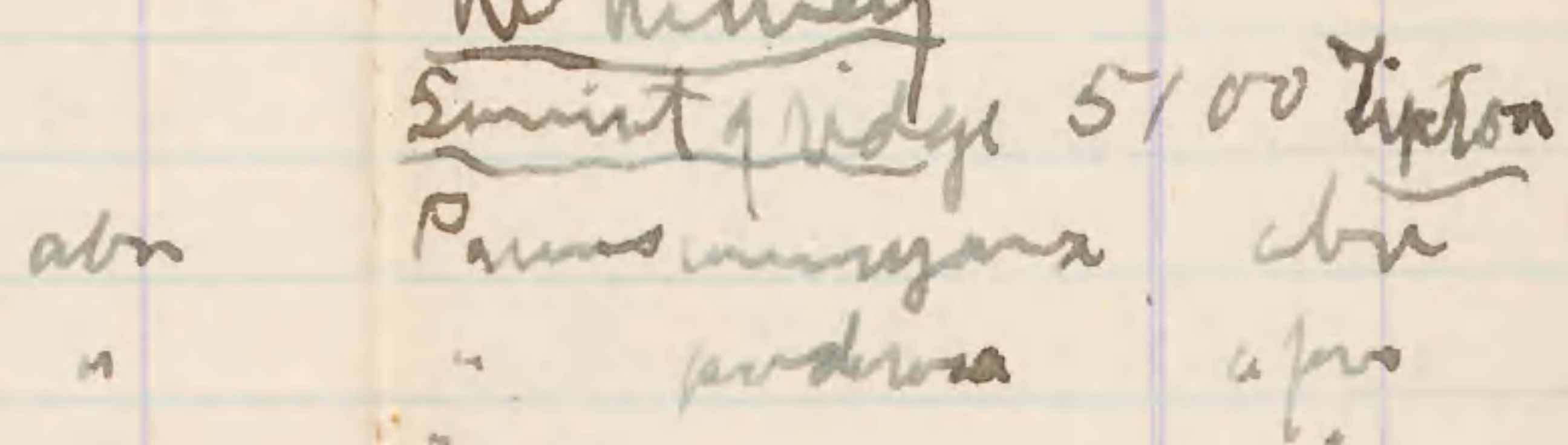

tavix chn

v

apas

Prudotruga

ouphats Populve Tnumbing

u 
Anstin 4200

Imizs Campon pot5 525

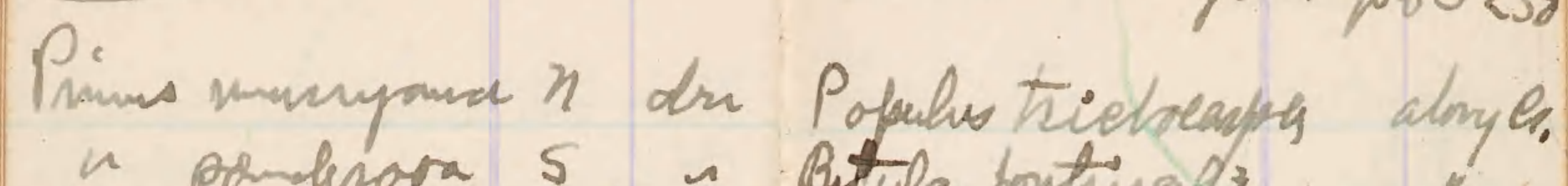
" ponderora 5

Zarix

Poundotonga

alics

aluns

Solin

Vincthom

Ropen

Sorbut

Cieovith

clanty

sheplise

Pave

Prinis

Campon lity. ajus Alume

puld Salix

" Pinm pondunat -5200 it

aloma es. Psucudolonga

ifferus arcihotehs tonss.

thempos hidplins

ingic tidoletw

Envis triduntita

unpothamas quary.

is tastopu?

ustomarrtinga

whines nolanreapa

ieladiphis linini

'arleia 


\section{State Musel}

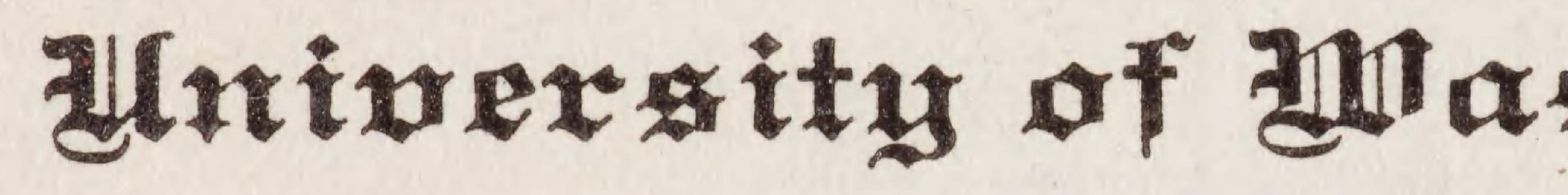

F. S. HALL, CURA

C. J. ALBRECHT, TAXID

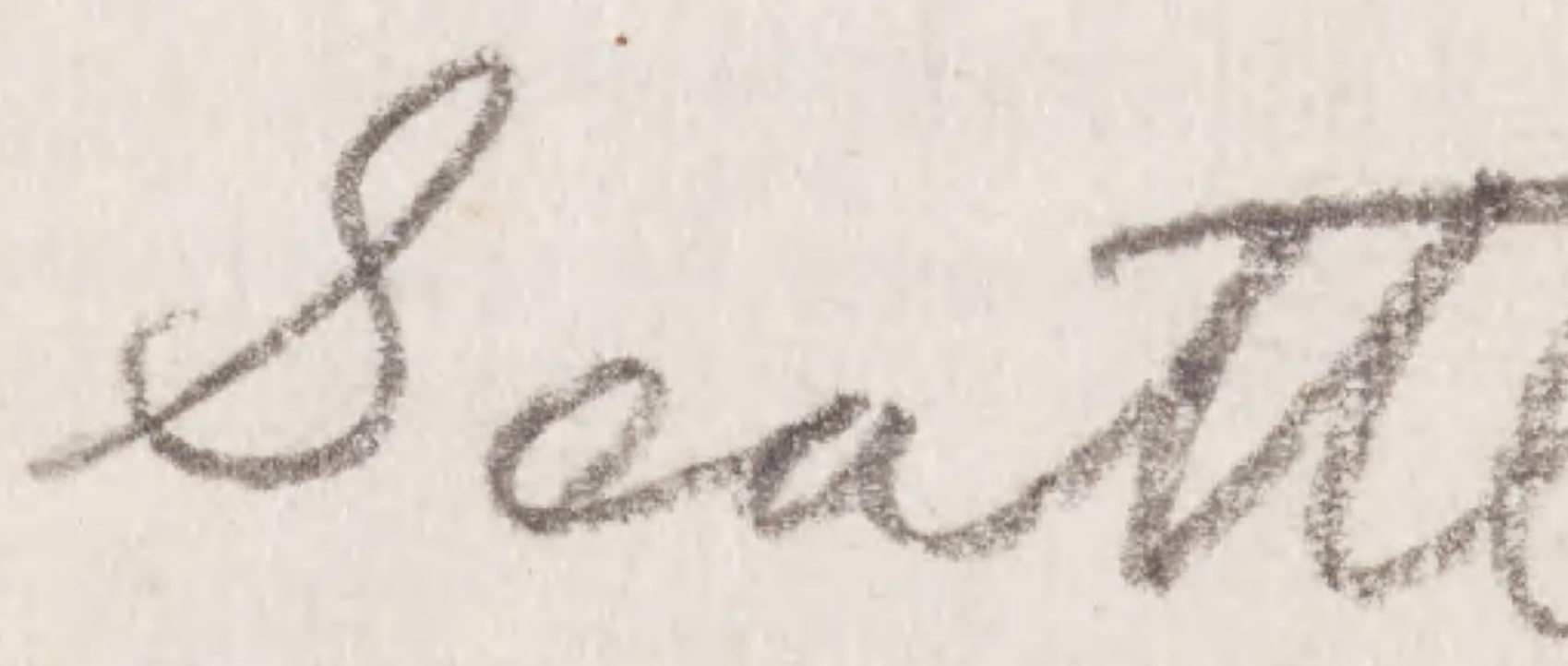


Anstin 4200

Jumzs Campon pot5 5250

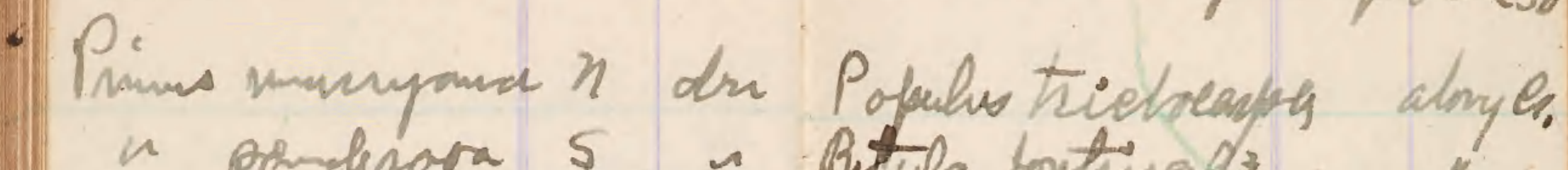
" pondirara 5 in Butula fortivads

Paric atuo Alume

Paudotonga upinled Salix

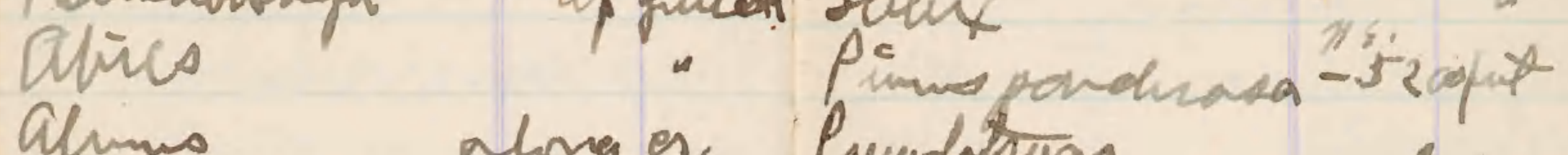

alims alona es. Psundolorga

Volnotrum

Ropen

Rutas nitkams

sobous

Erevition

insitivasia

Clangthes nelutions

Sheplestia candonicins

Pasa-5200

Prairia 0420

Campon loty 3150

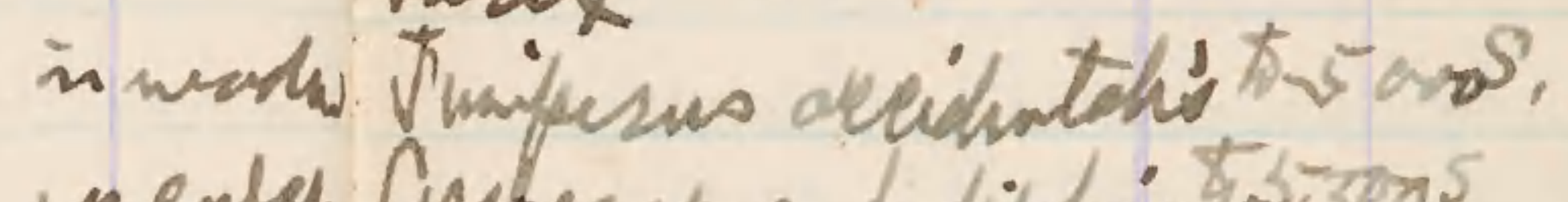

up guleh Crefrearpos hidiphims

Kinuzias tidolets

" Getrunis tiduntata

"Chripothanas quary.jon?

Aelstamantinga

Wyithia

Sambues wolareaspa

Pliladiphis luvini

Clerleia 
Cumplaty

- Depquehas

actitis

Aquila

Faleo stensins

Zenaidura

colaptis

Gsindirmys

Inpraminot ty

n visticalio

Somponis anyi

Enpidonax.

Coteppos vichandini apos

Sitta mymaia

Cosvus an.

Euptrigus

aflains

Letems vallarlés

stirnella inglita

Piranga hidorieiana

Firundo 5 yg.

Dudprica andobris

spigula arijonas.

Pasacaira anorra

Mulampiza

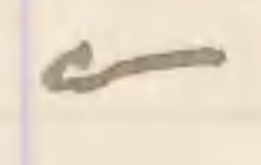

con Elundoto

- Cantodaces

1

pipilo

1 Passer

en Salpinats

- Percus

In Planestiens

Sialdín cumneids

"Sudriva sotios z

c

heaed I

cu

2

lueet.

e-

w

2 Seinnes

ontwis.

no Ectanias

- Callerpenophibes

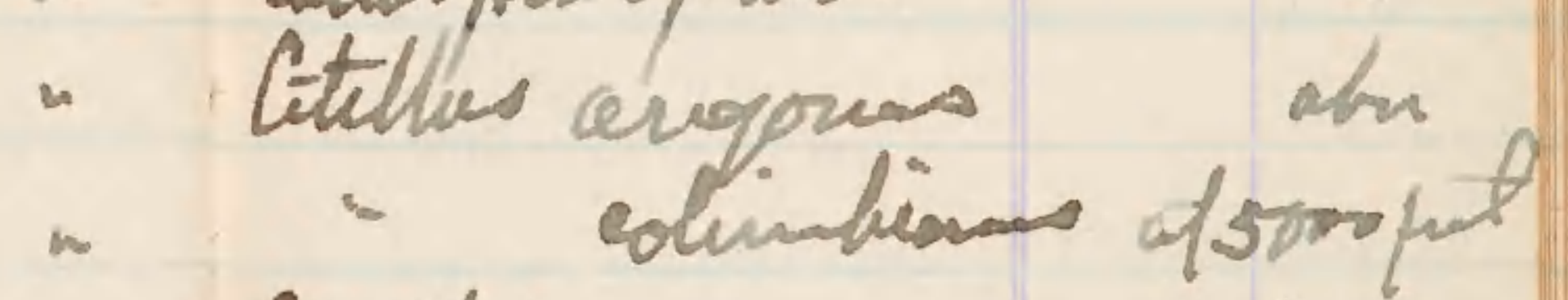

n en

- Eectuion

n Thonngs

n Hepene

oblignas

b iypilapres nuttedi. I ans.

- Mutoma

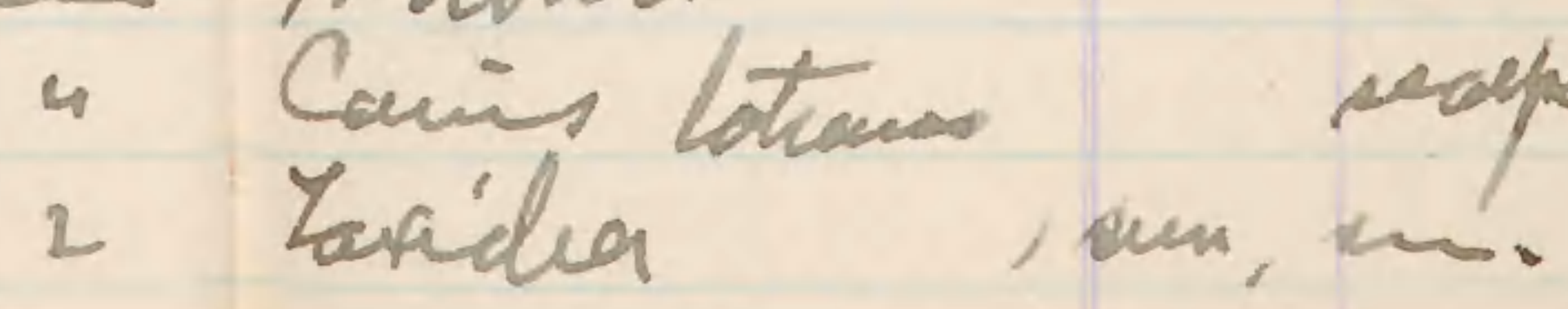


Tinz 27 Prairi,3420 Strewherm Buth

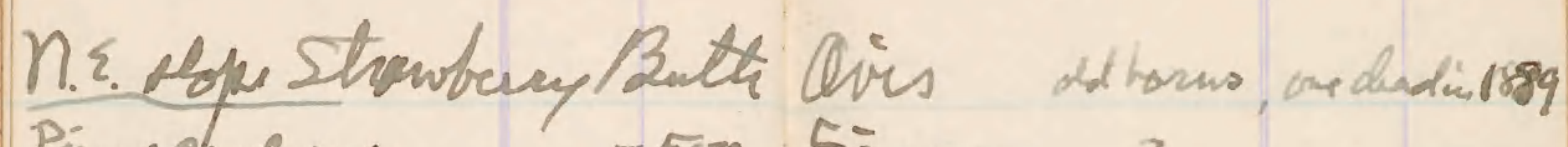

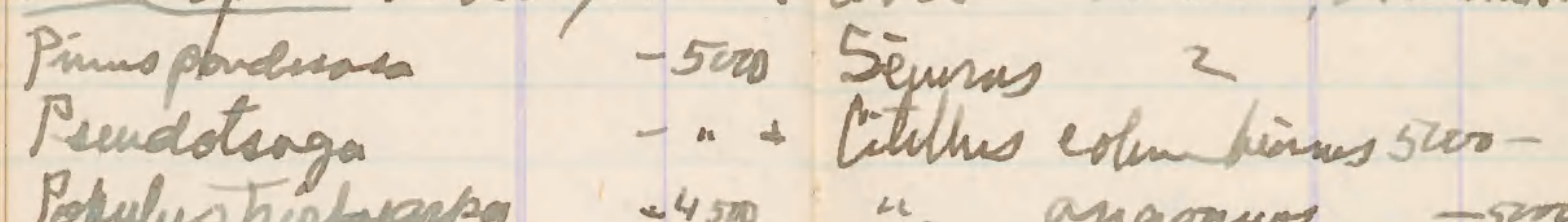

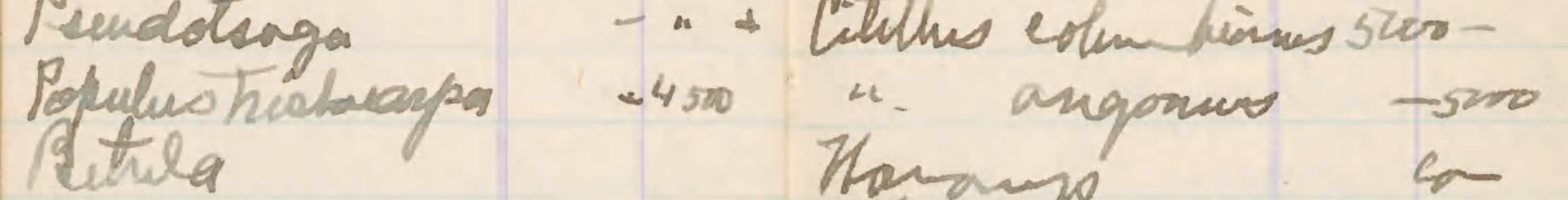

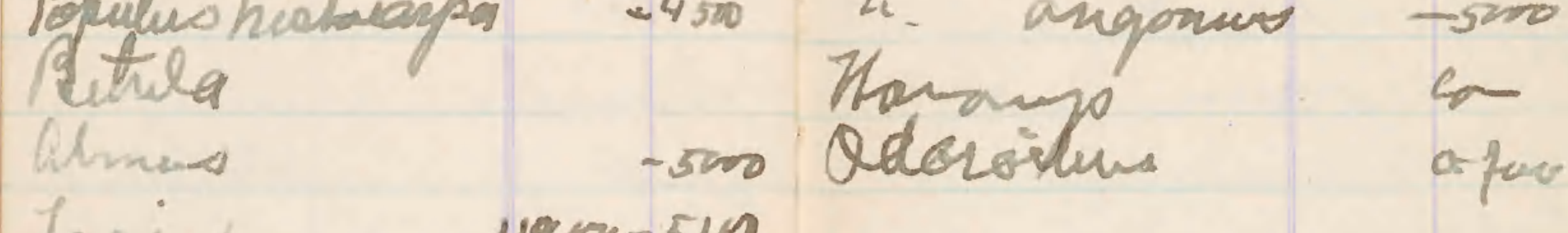

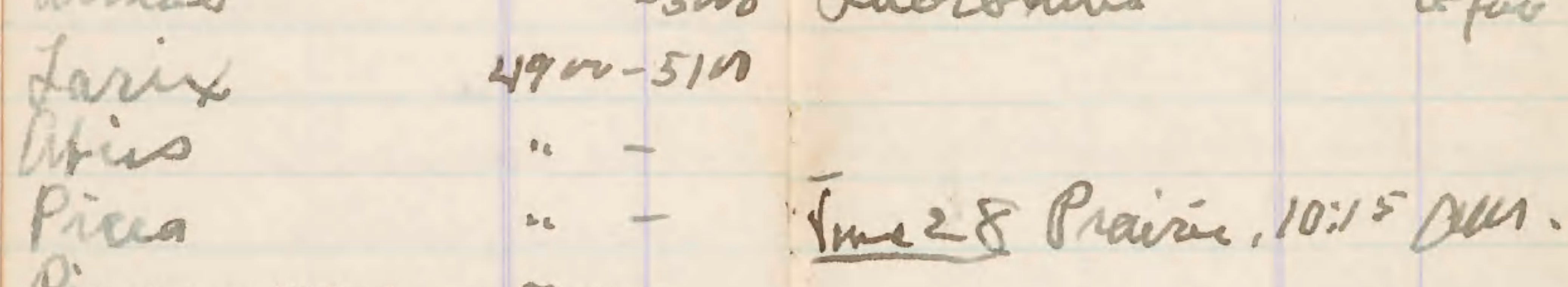

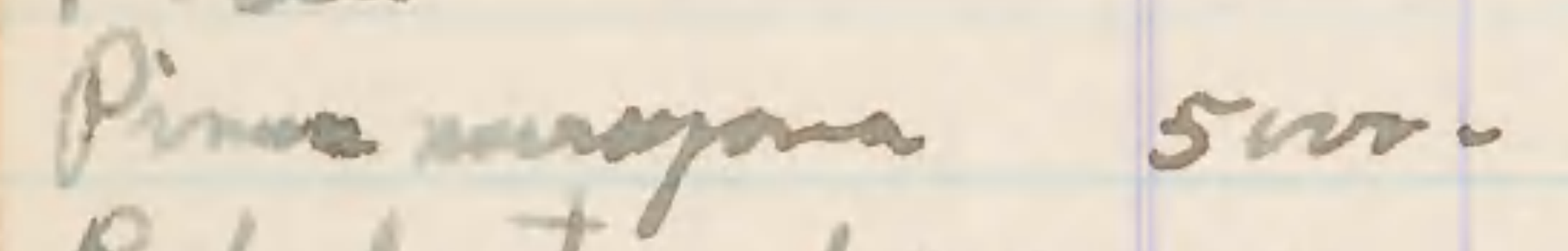

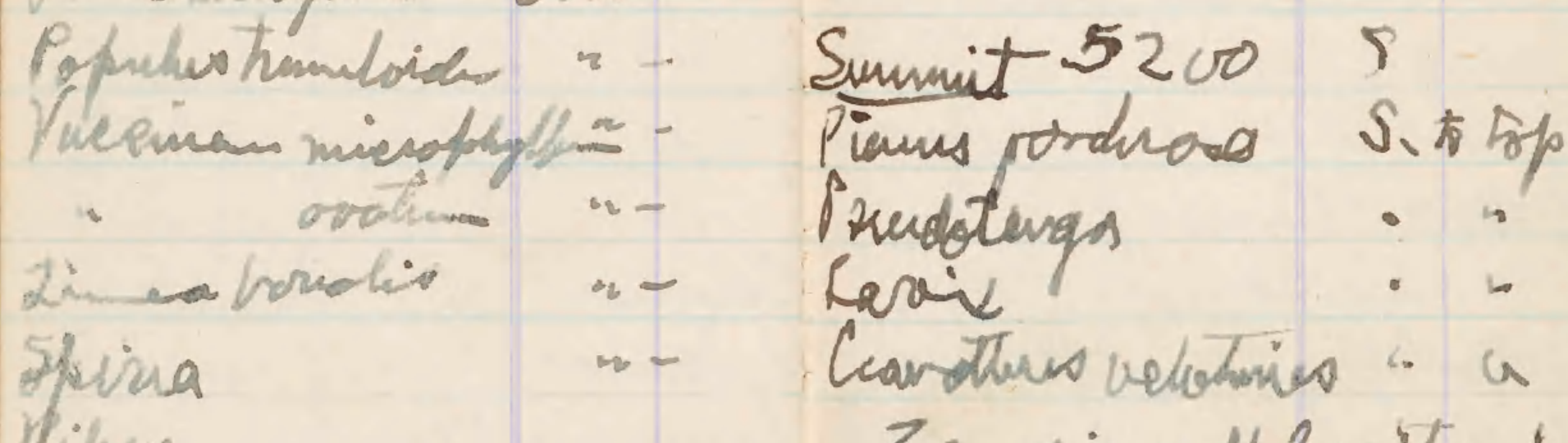
Nibe

pravulaina Zenopiens alboleswitis 1 X. Slafel

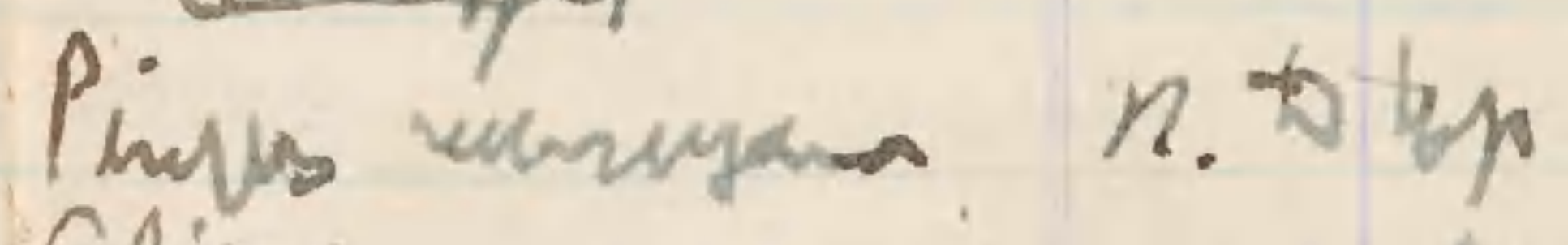
Jumecos Hylievda Cabins Pieca Pirmega lindo. Msundatteng Piea Vacriven wa

coppen 
Simpter a Borns

Iumples " Ram

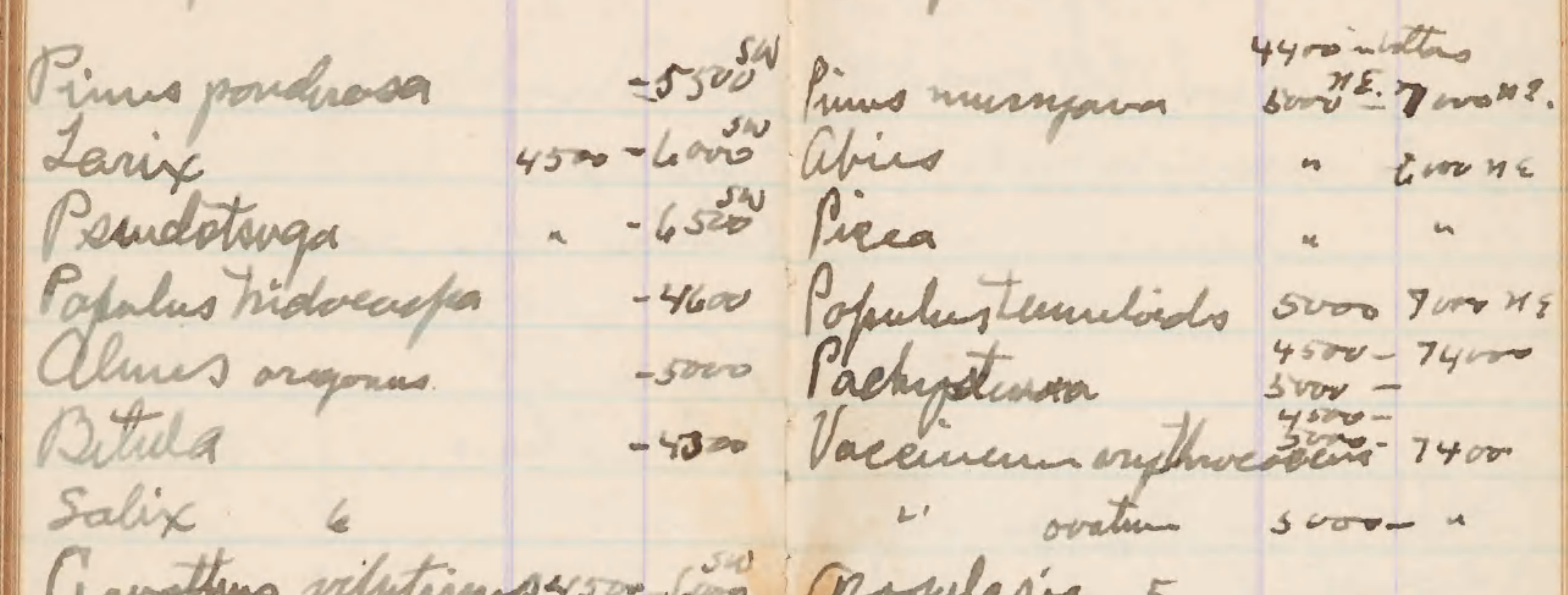

Gavathens vilutimosson-6io grosularis 5

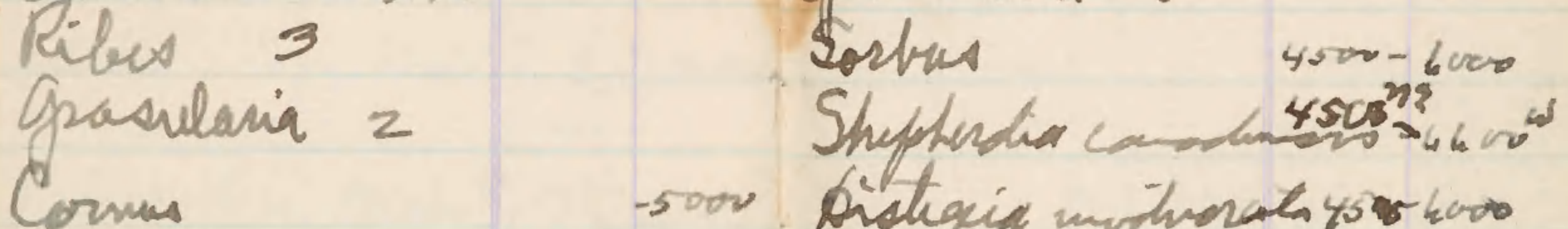

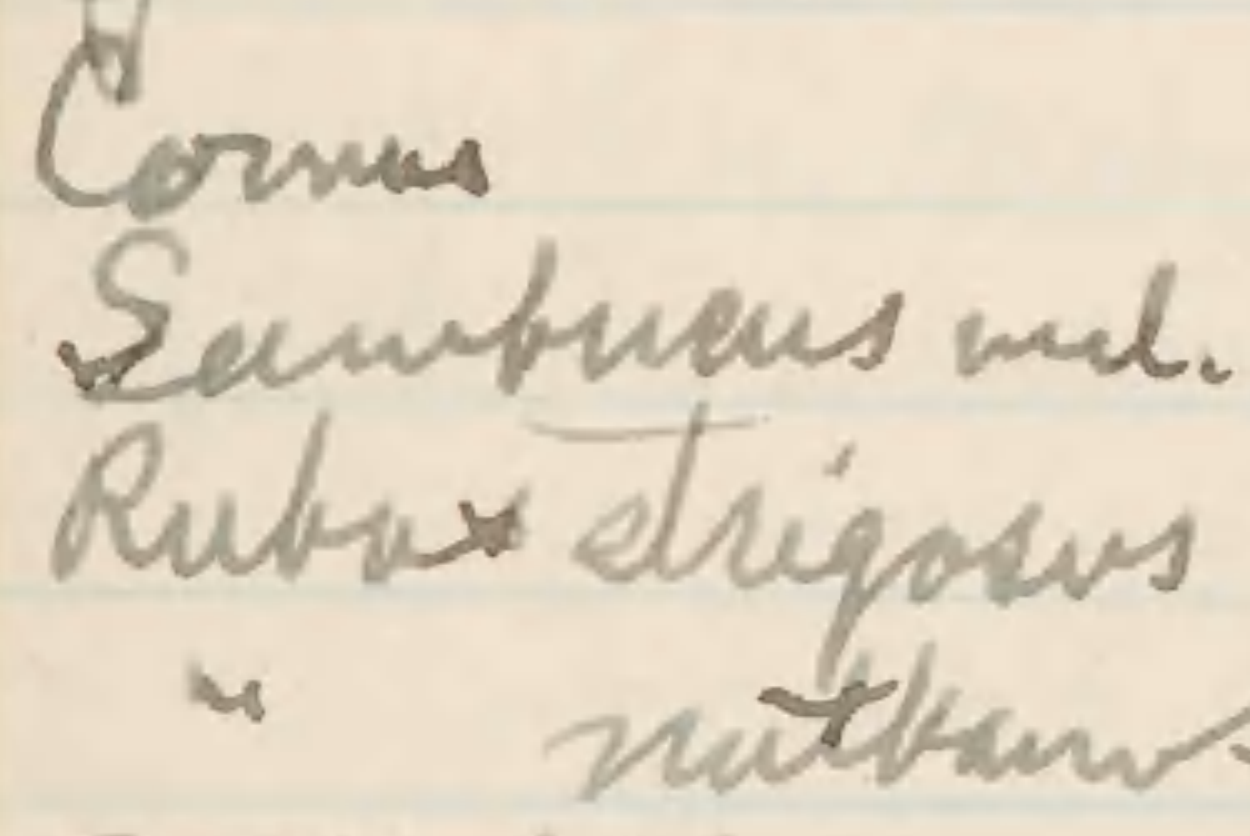

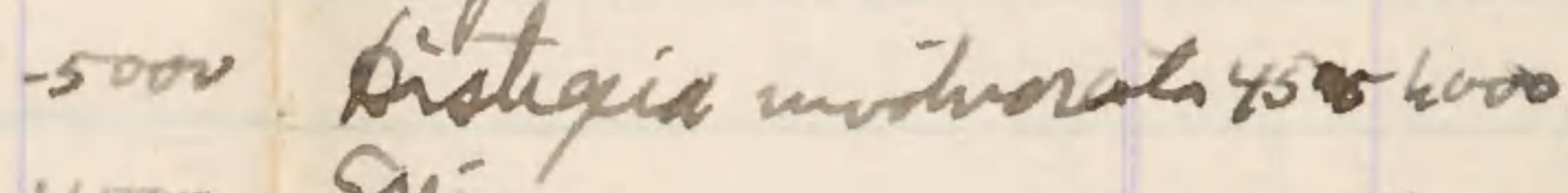

4500 piria

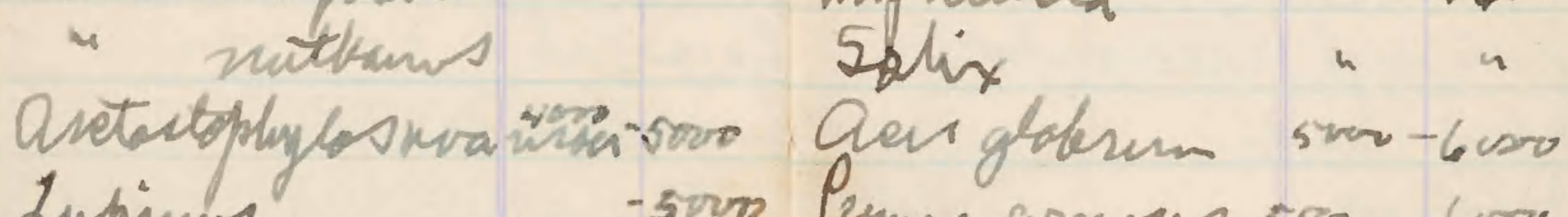
Lupinims

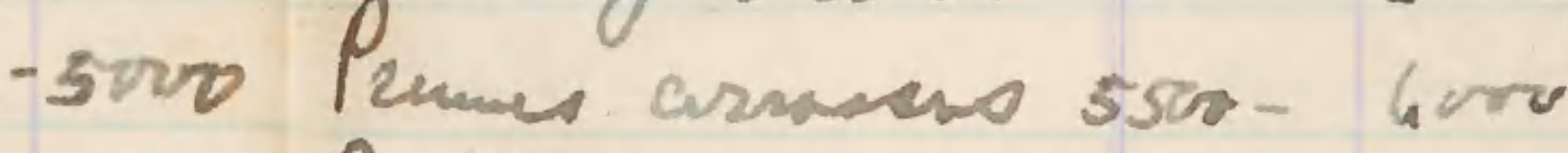

Suchum

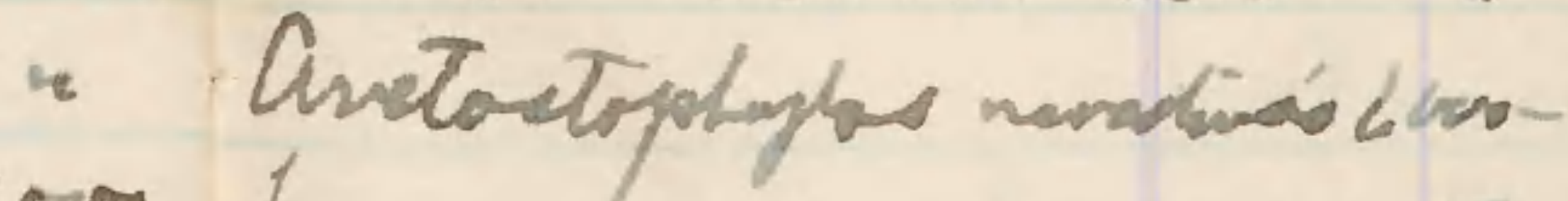

Besbins spens

-3500 tomeracangapistis? W00 - Tovo

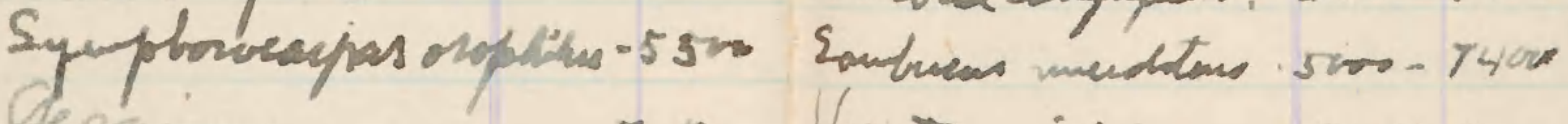

- "s Veratremsinden jover-7ro

curtallya

- 5 mon "spunqu"

$6000-7000$

Ereveagper bodiplies

$-300$

Palmoniman folidorm?

$\therefore-7800$

- Olmus.

$5000-7000$

Antren

Valurian

6on 7400

Qhis Expresta

Pins allicentis

$$
700^{2} 2
$$


Sumptrat aboum, ju 29

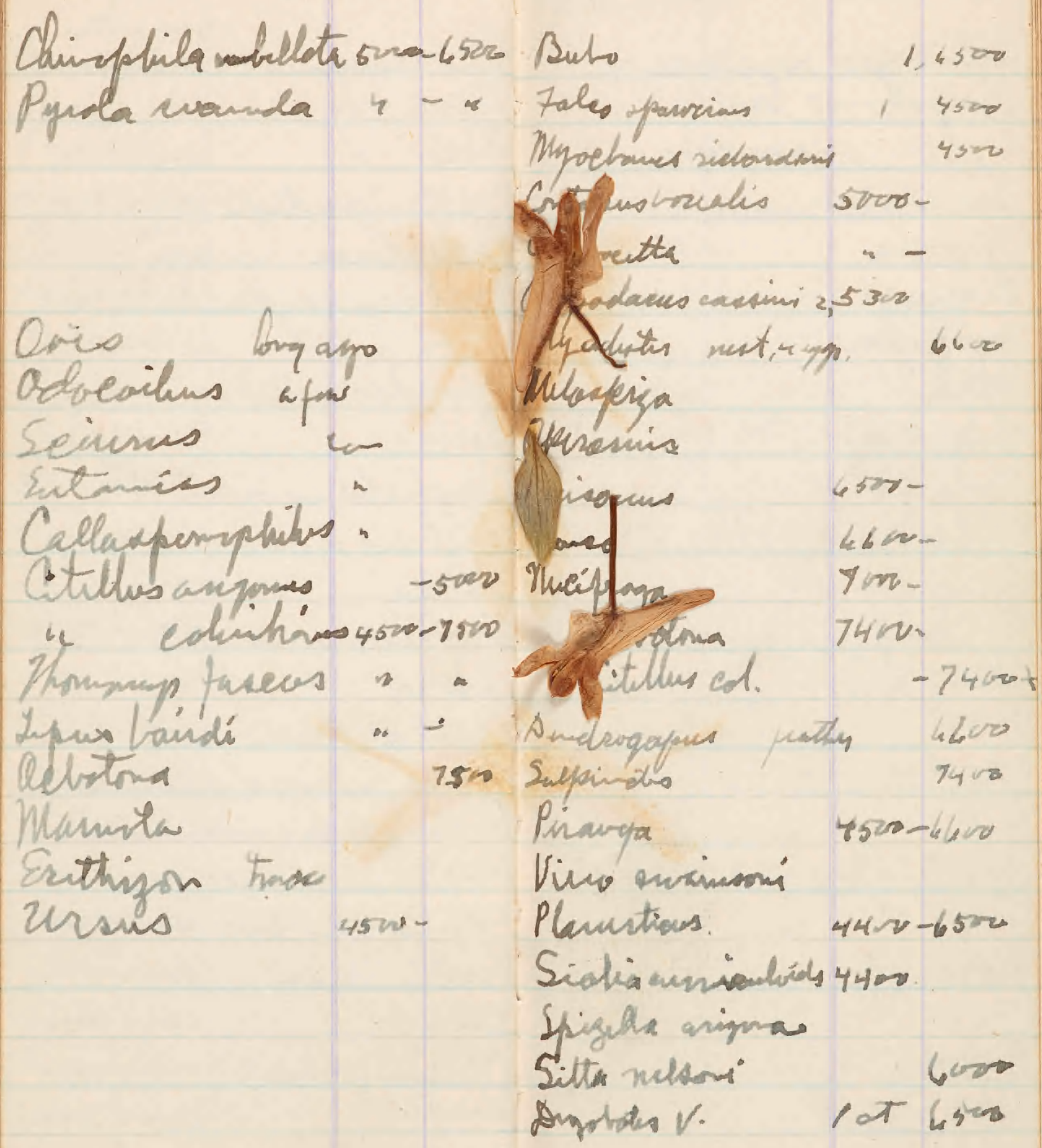



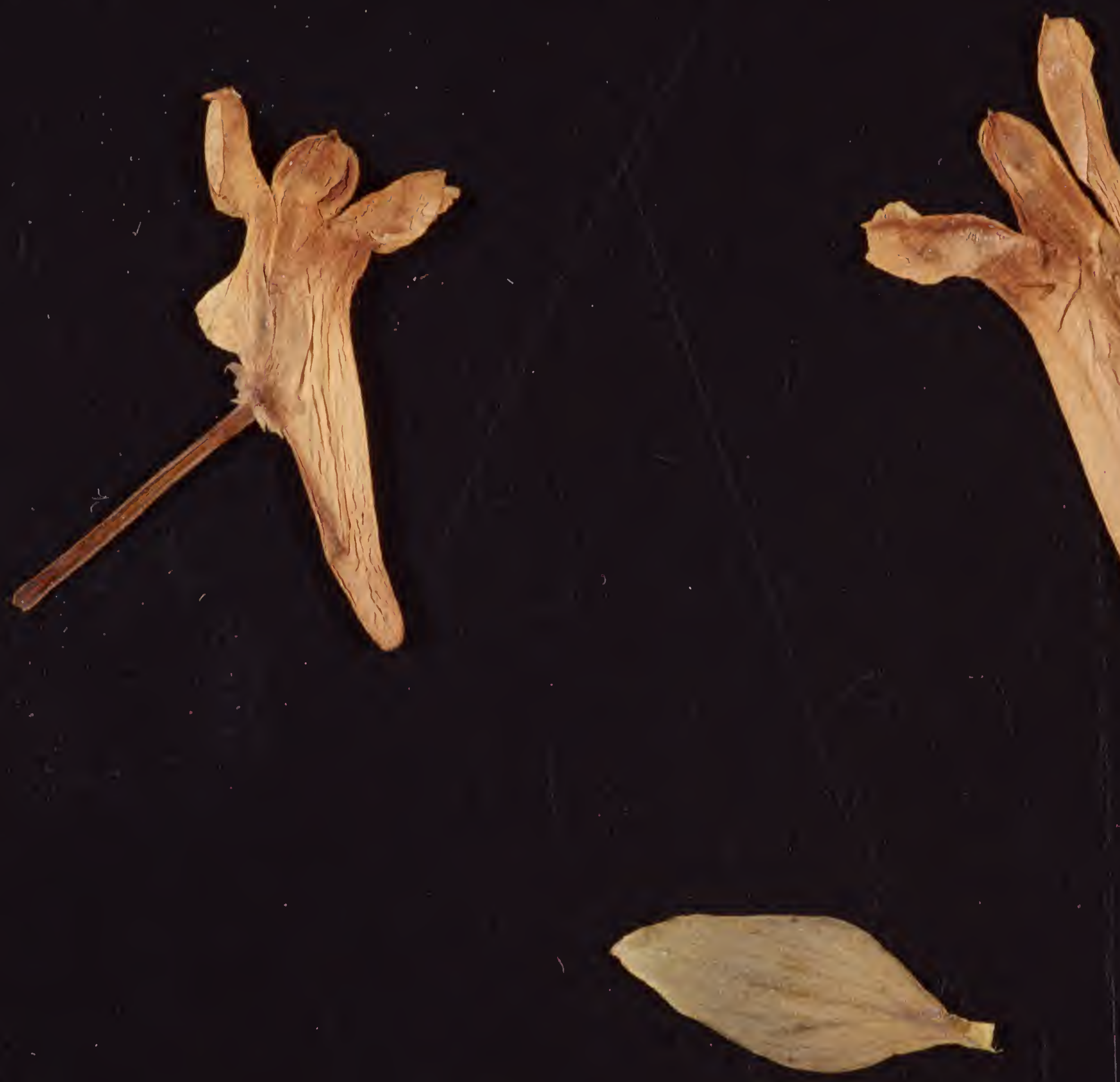


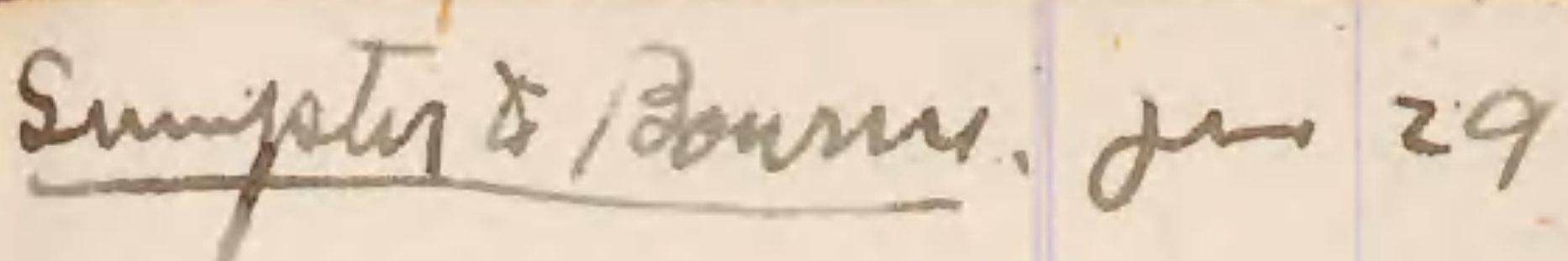

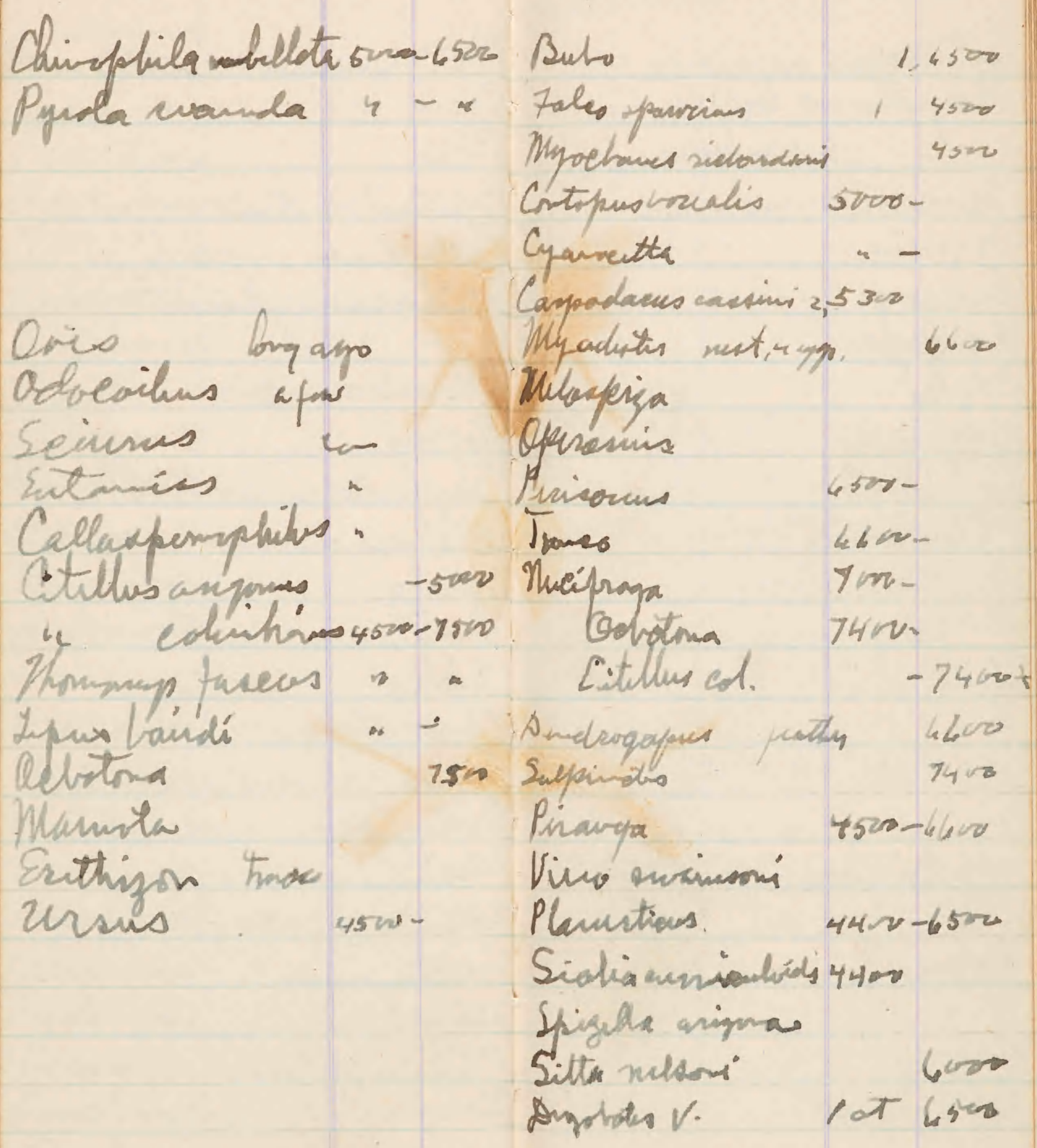




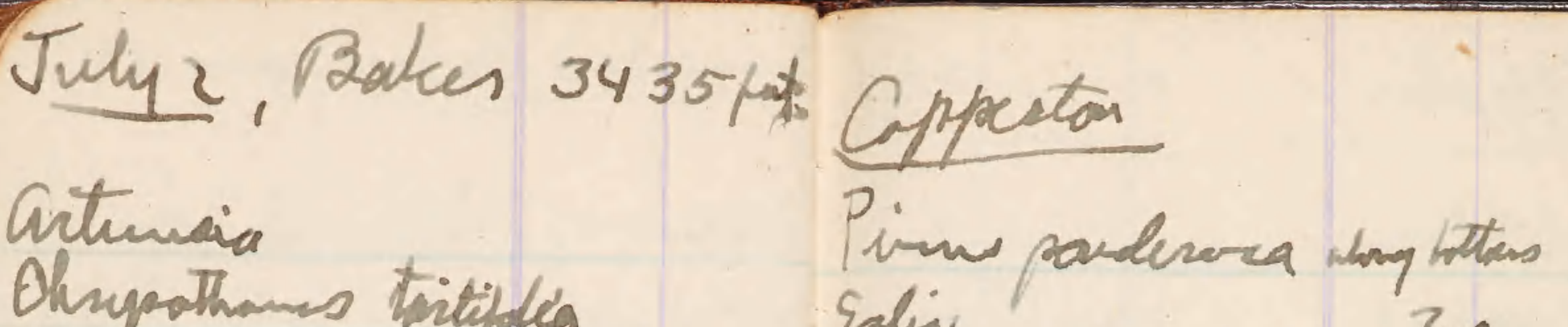

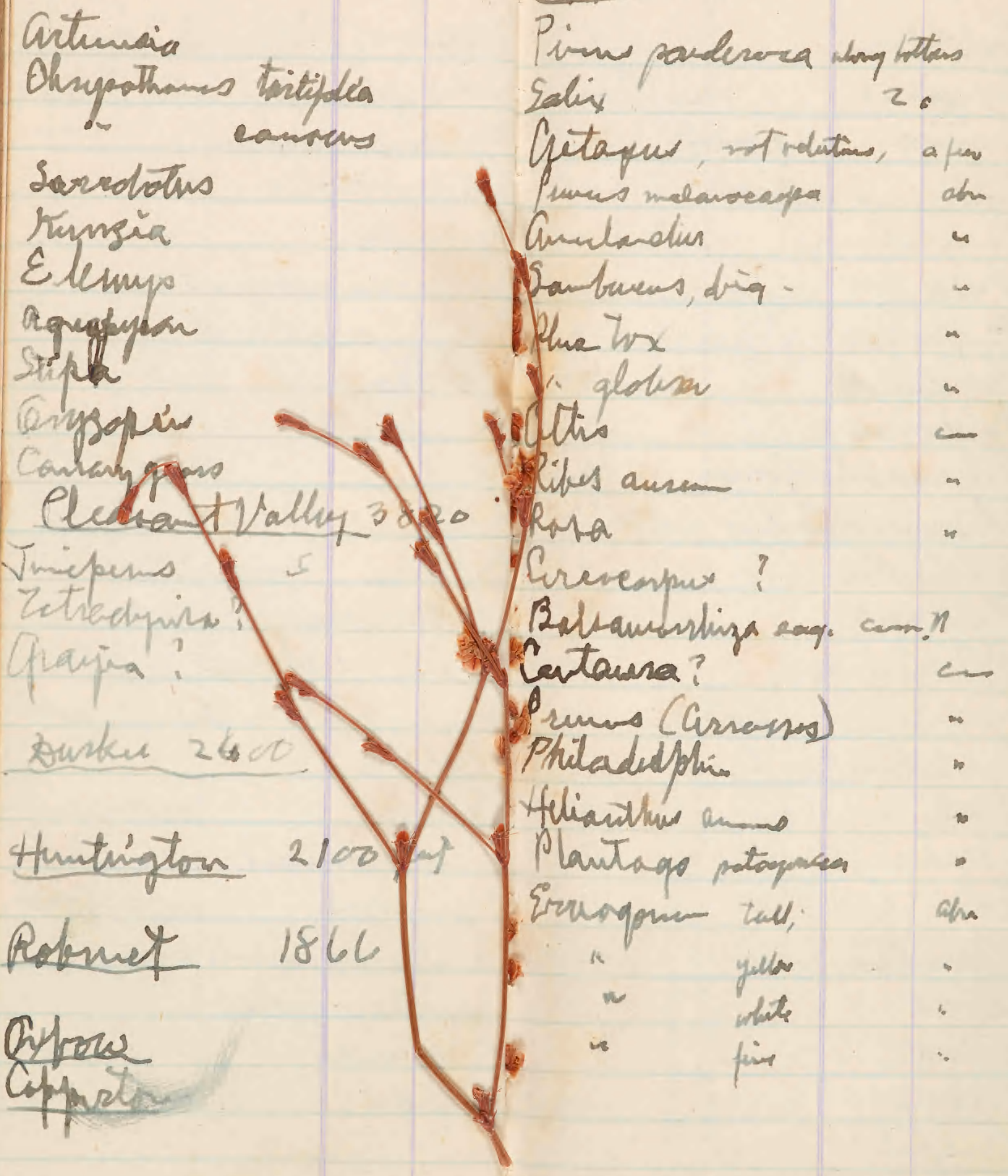




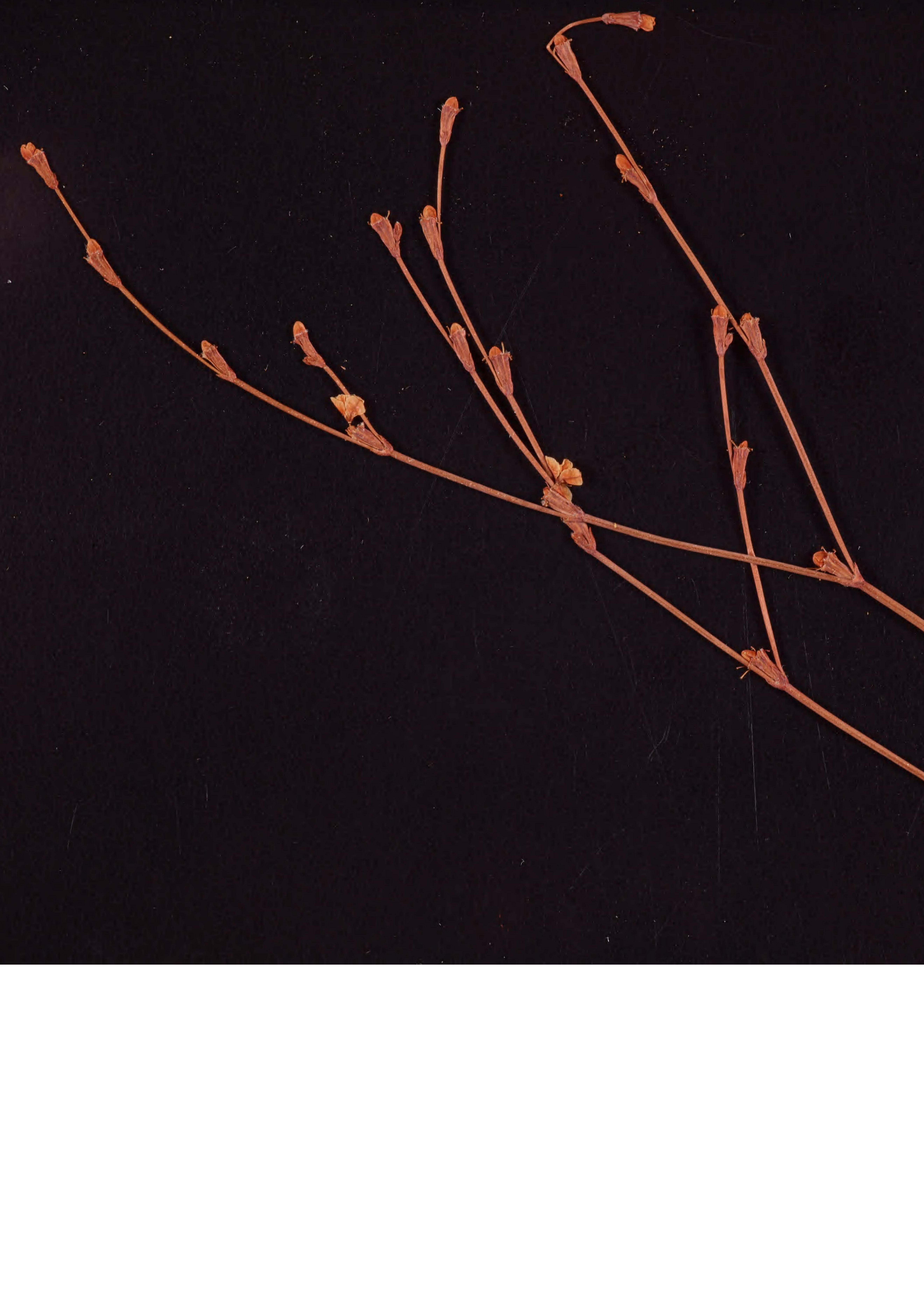


July2, Bakes 3435/4* Cppestar

Airturaia

iven pardererea ung withers

Salix

2 .

Getapur, mindution, a fur

Saredotus

rungia

puncs melavorappa

Anualandiss

chn

Elempo

Sanbuens, dirg

aguafyian

the tox

arysopin

citiglodisa

a

concug grass

Rifes anseon

Plevact Valloz $3820^{\circ}$ Rra

Timiperms

Creveaspux?

Baltaunashing ray. corm.n

Cartaura?

Prums (Grrans)

Philadeiphin

Heliainumas anano

Huntrigton $2100 \mathrm{far}$

Plantage notapancas

Erruapoum tall,

Rotemet 1866

Cypow

" yeus

$-$

,

$-$

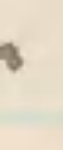

r

Bunkue 2600

" whe

copprite.

n fin

$\omega$

$\infty$

n

พ

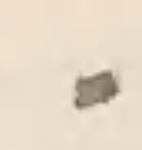

ahe

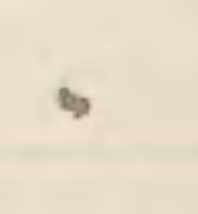




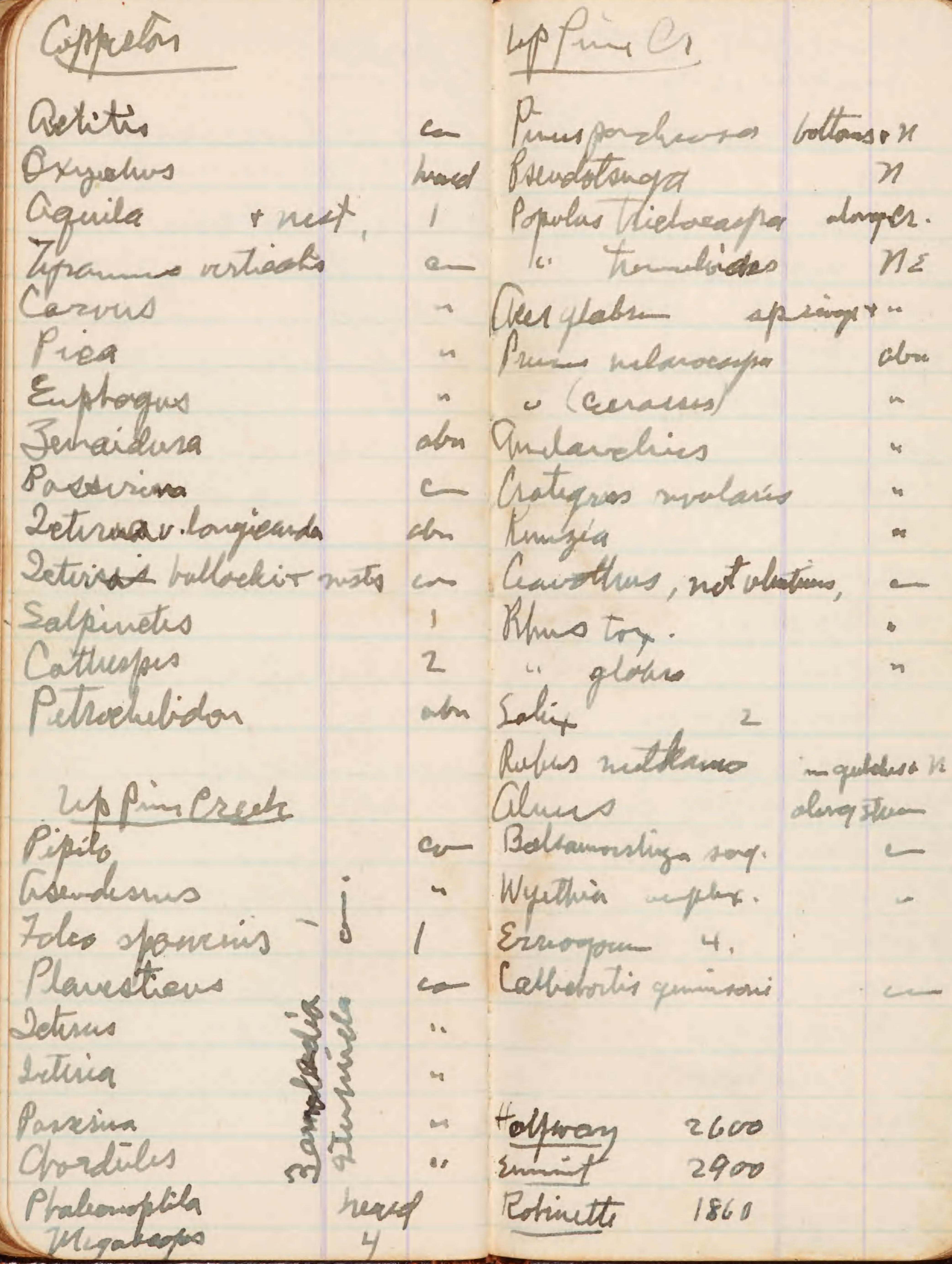


Carmerpia 4600,2 bs batinereat 7300 :

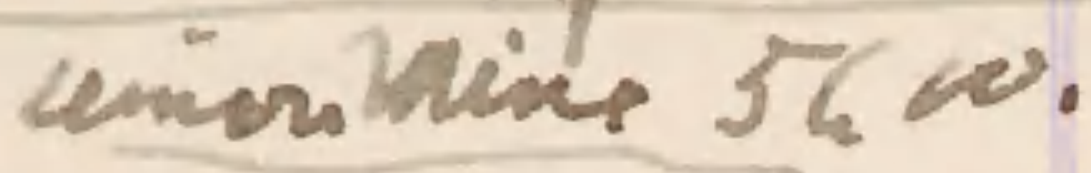

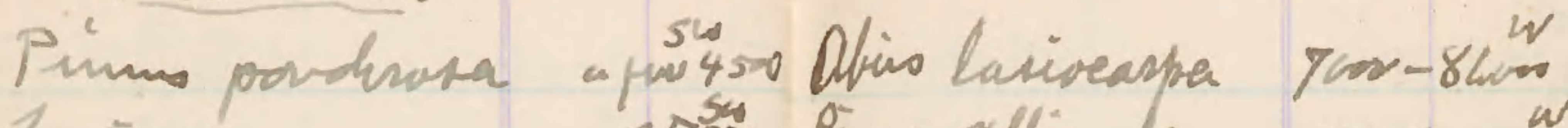

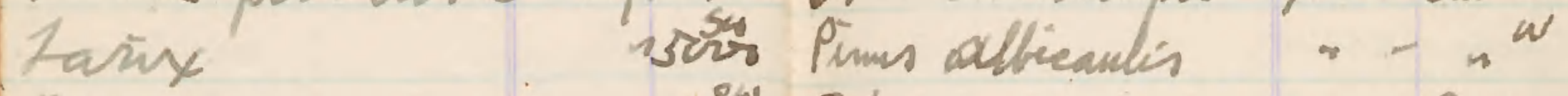
Peuditang - $46 \approx 0$ Pdyogom

- Sere Abies ancoler

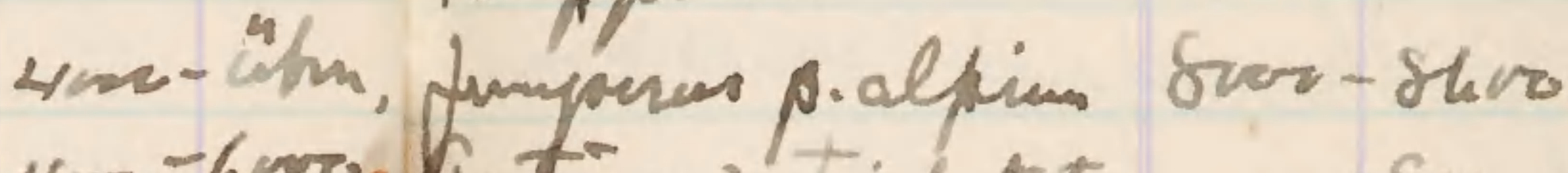
pícua

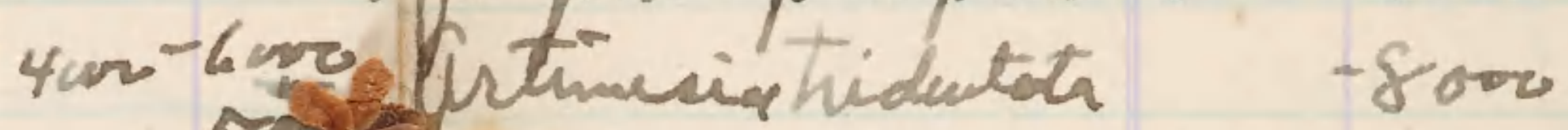

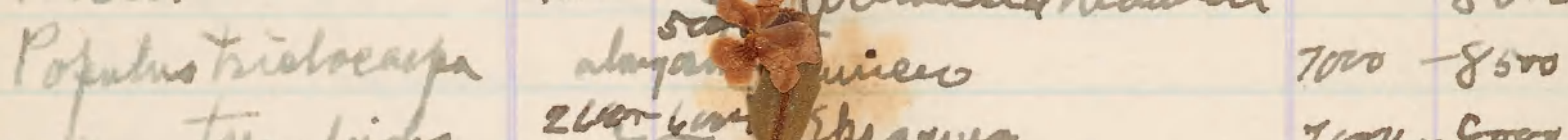

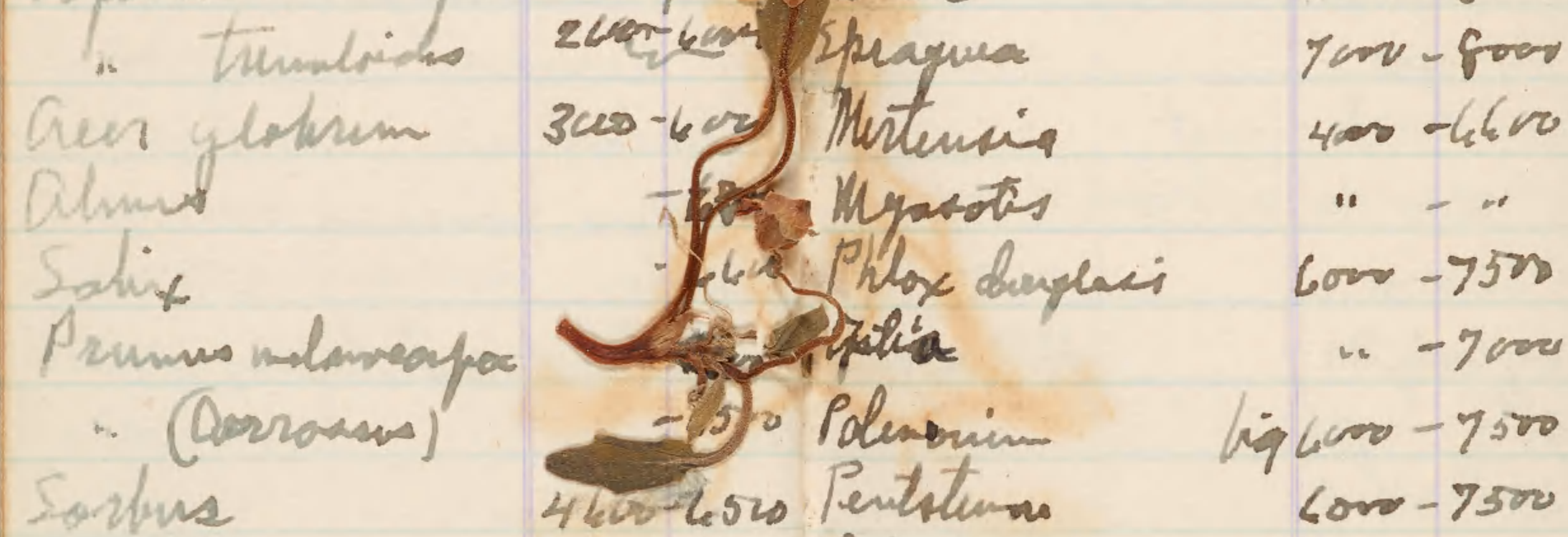

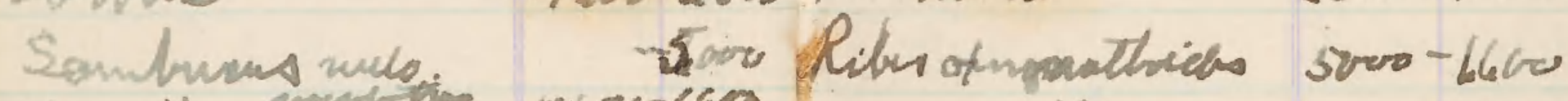

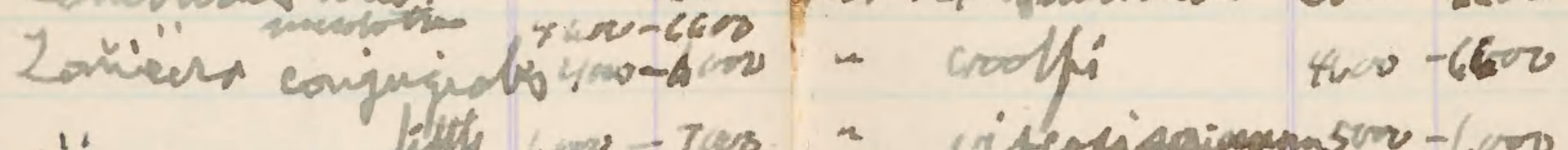

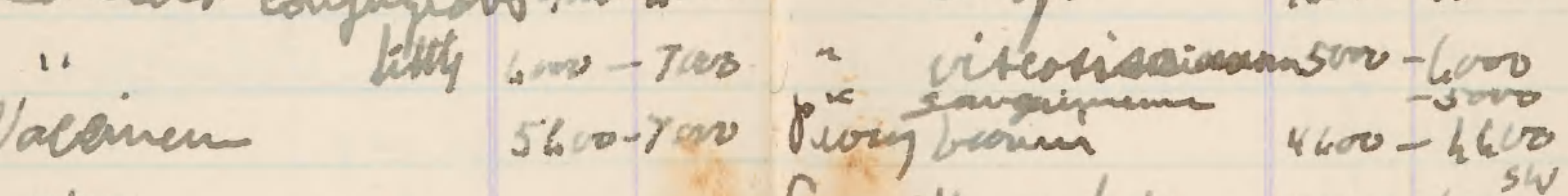

Veratrma

Ceanothers prlution

$-6000$

Retus mithenso

$-5000$

Valivion

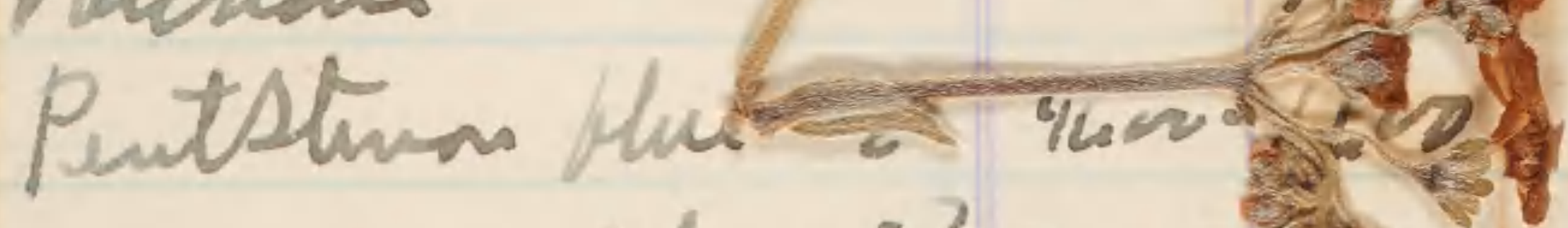

Ballewanshizg

Wrethin

Suphororespos

-4500 i.

-5 ins 

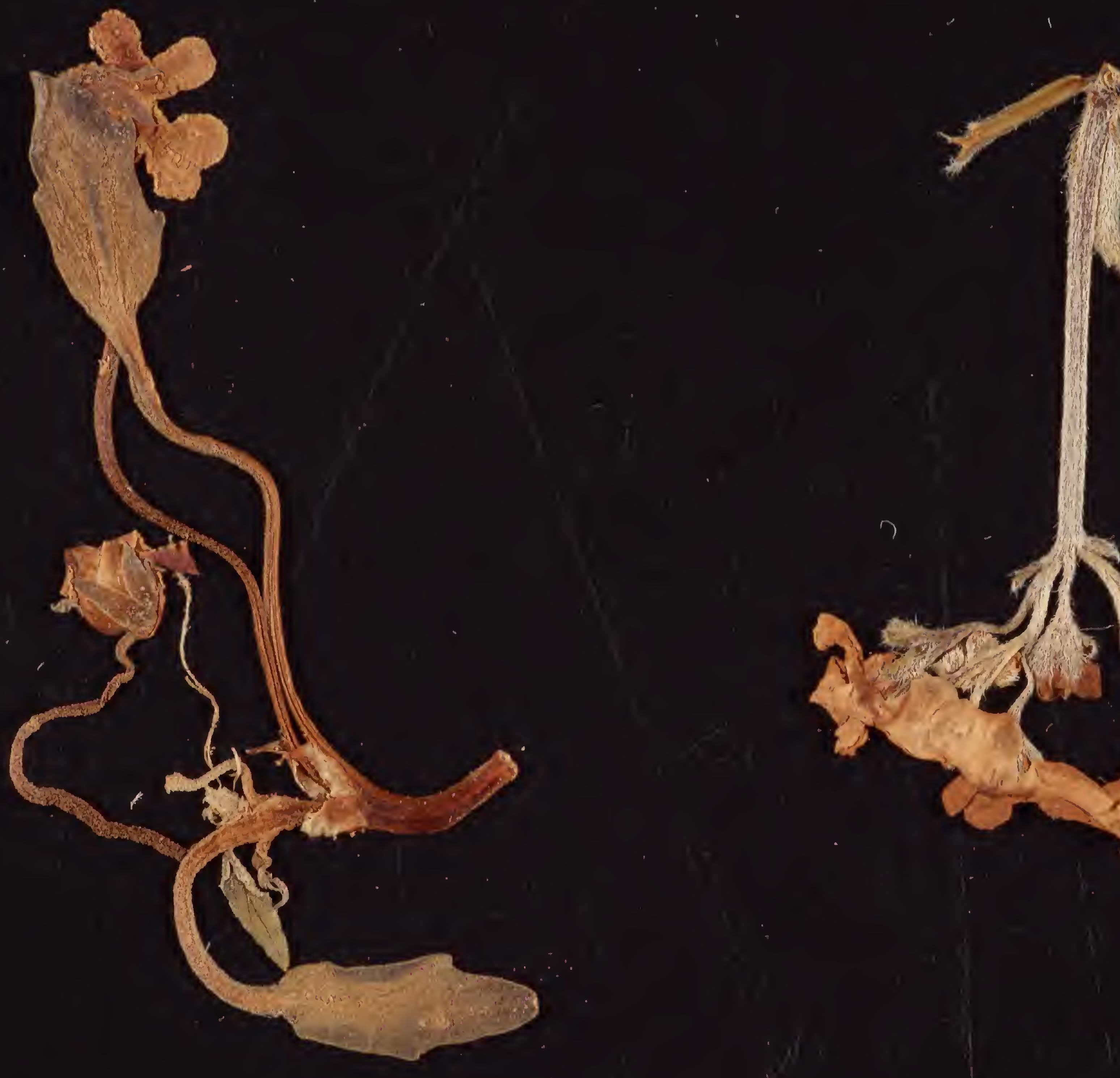

Carinerpiar 4600, duds batromeat 7300:

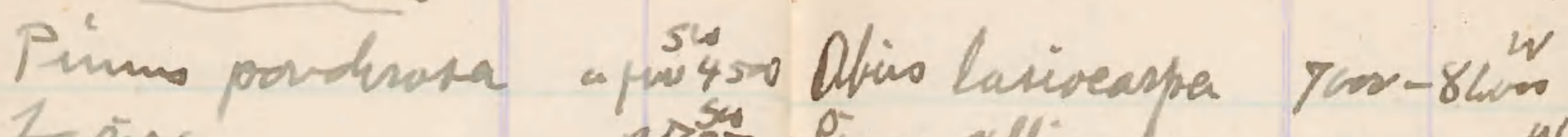

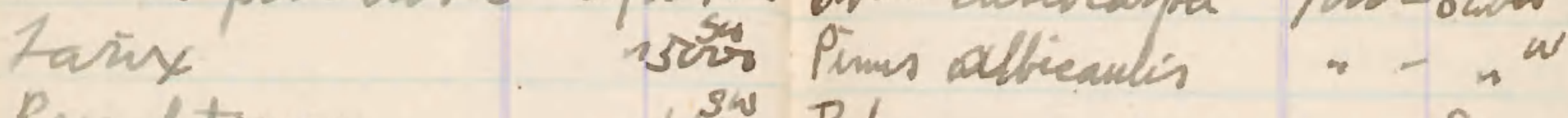
Peudoting $-6 b^{3 i 2}$ Pdyopom

- Sero

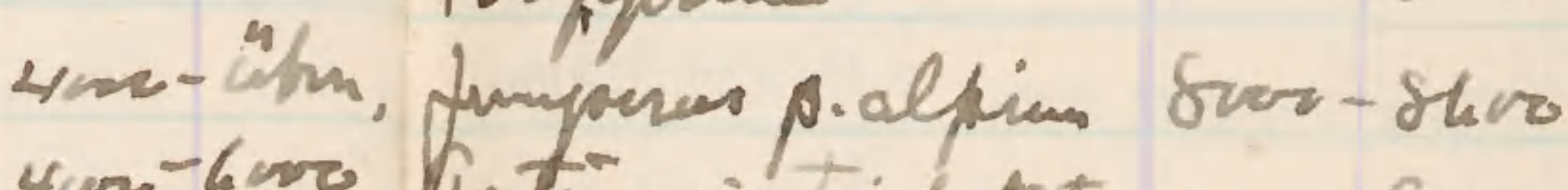

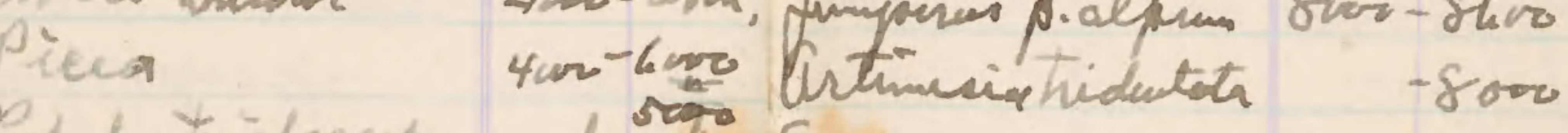

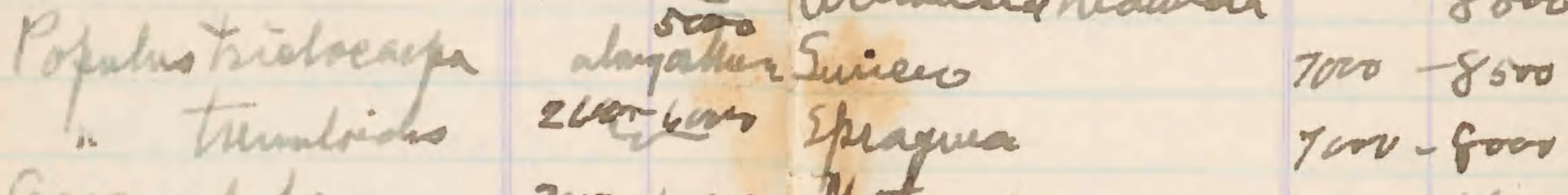

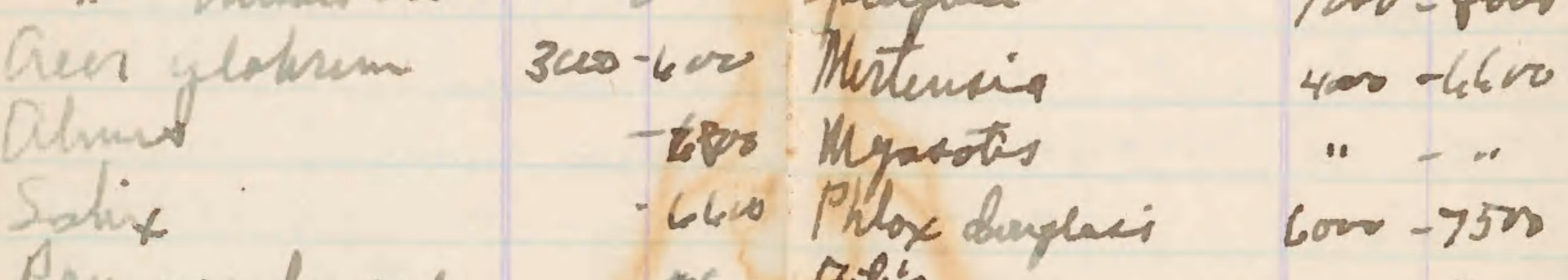
Prix minolaneapos $6000-7500$ (Corronsas) - tere Tatia $\therefore-7000$ - 5550 Polenorim $196000-7500$ 4600-leses Pentotumos $6000-7500$

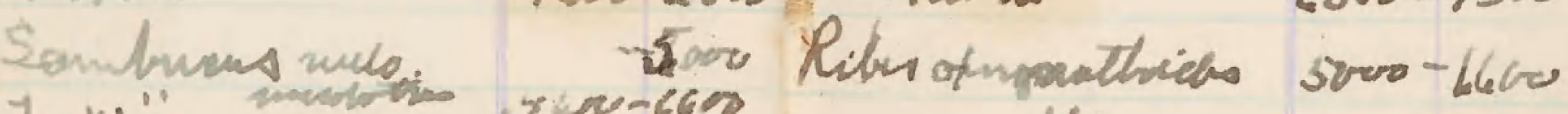

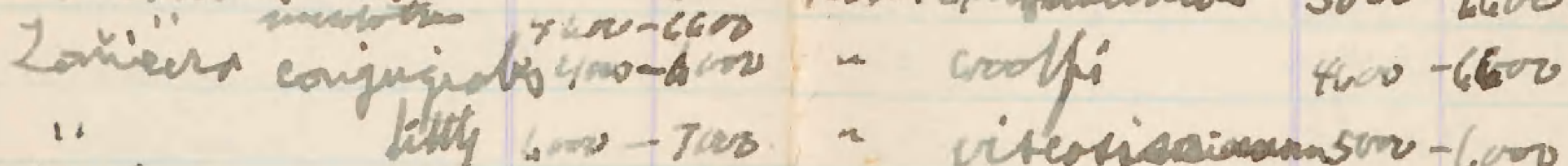
Vaceinem Veratrum

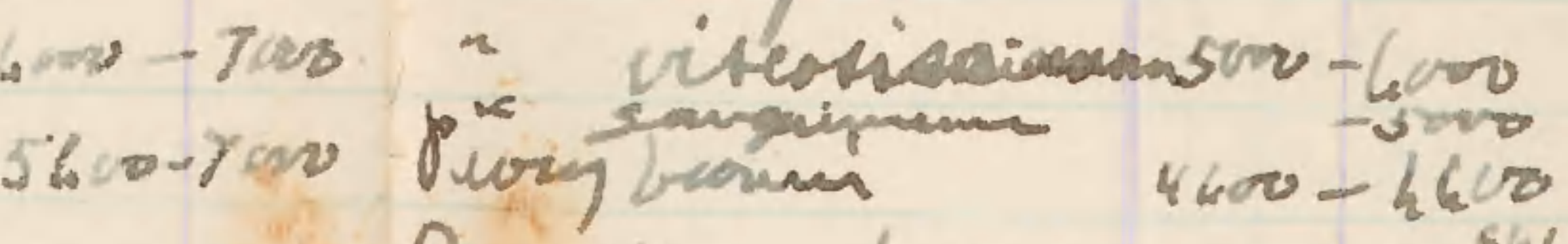
Ceanothus relution $4000-60$ ono

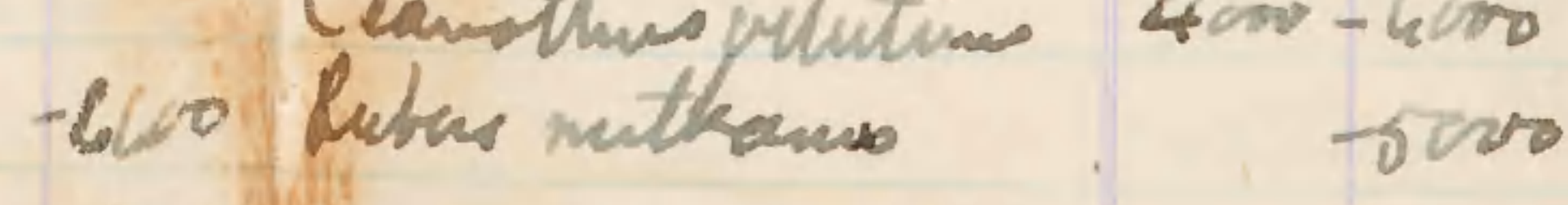
Volecian $-7000$

Puntstunos plue 2 4noverbos

" nusbermi? uno-7no Boltamanshizg Whathis

$$
\begin{aligned}
& -6,50 \\
& -500
\end{aligned}
$$



Feelyks. Hemtington

Antañor Vale fun 7.

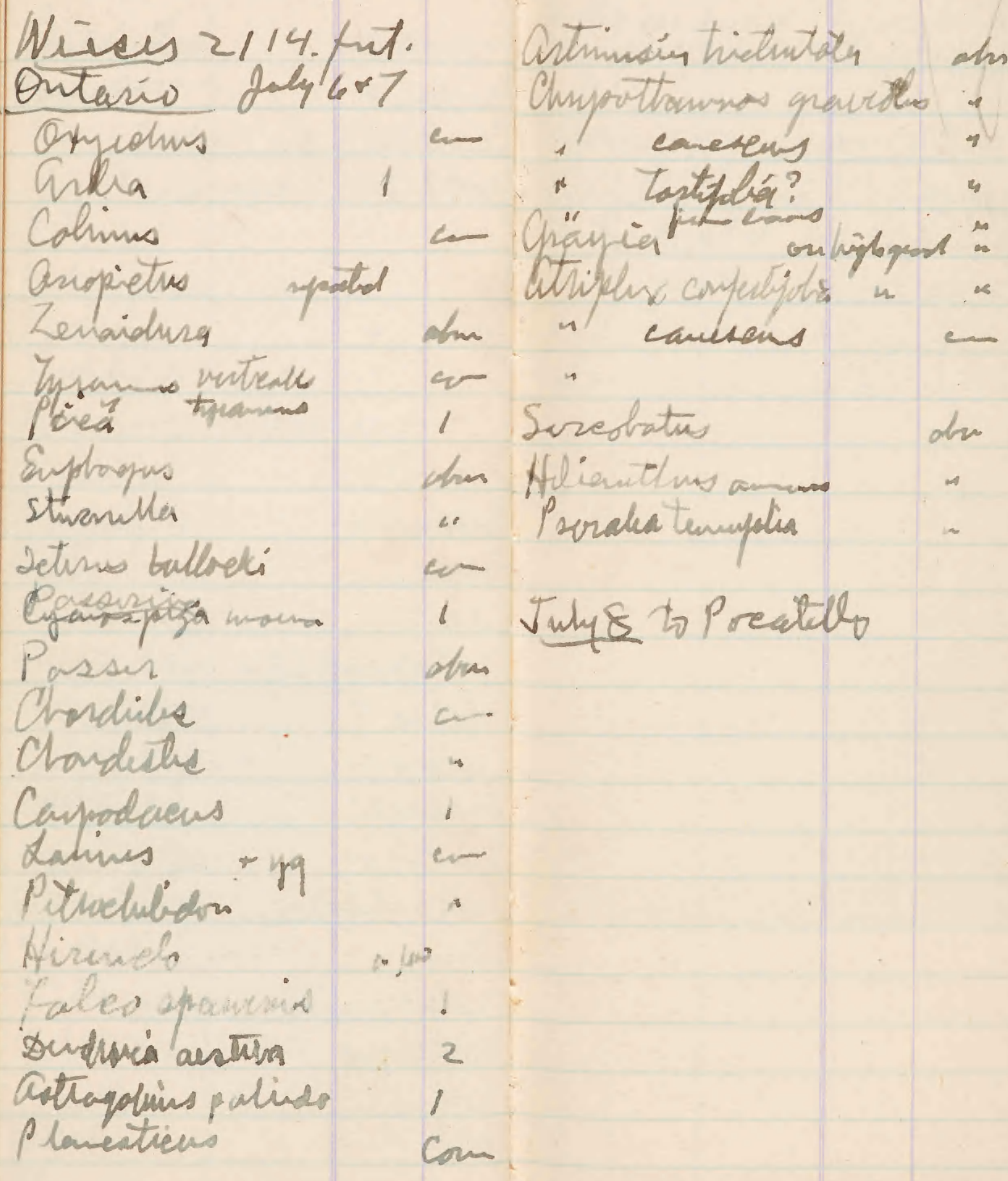


Copptespister

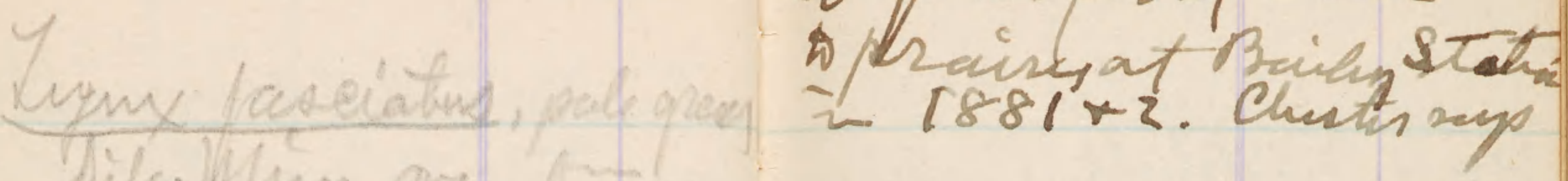

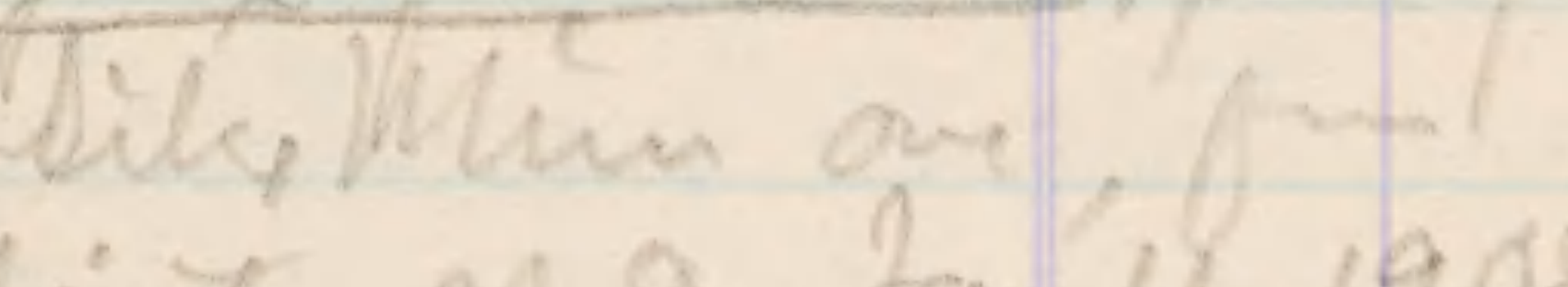

Mirito Par 12,1908 gray aquinstónest in

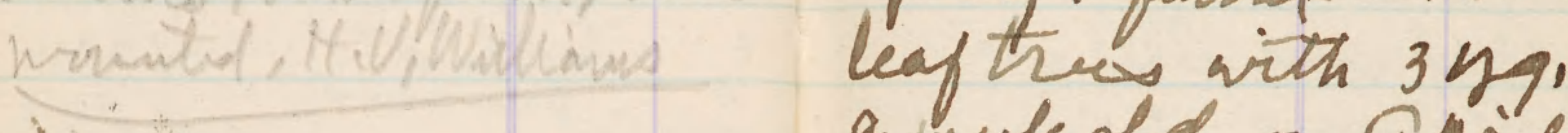

a mukald on Apintos.

Williston MD. D

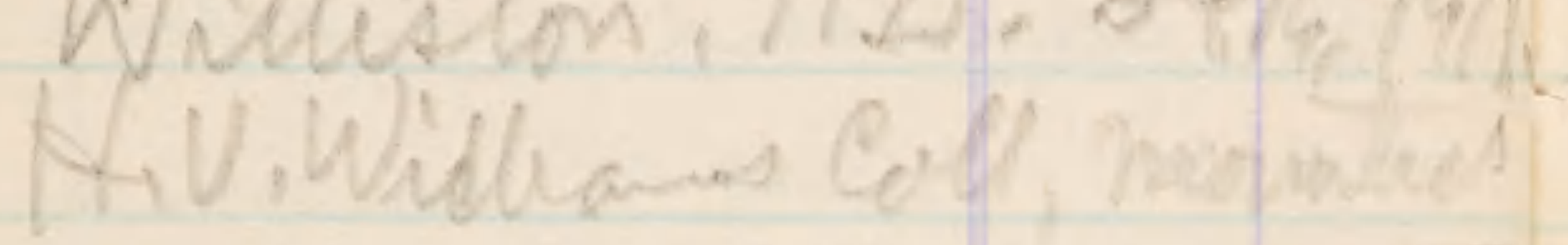


Bass 2 soing

Stiphersirth, Nory

sud circulas

Bird homu, N.D, te.

C. Q. Jones Tavidomust.

Boldm, Saskerteh. Pox 47

z. Axthilm, Bordm

Hoted perp.

ins .Marys. Clary.

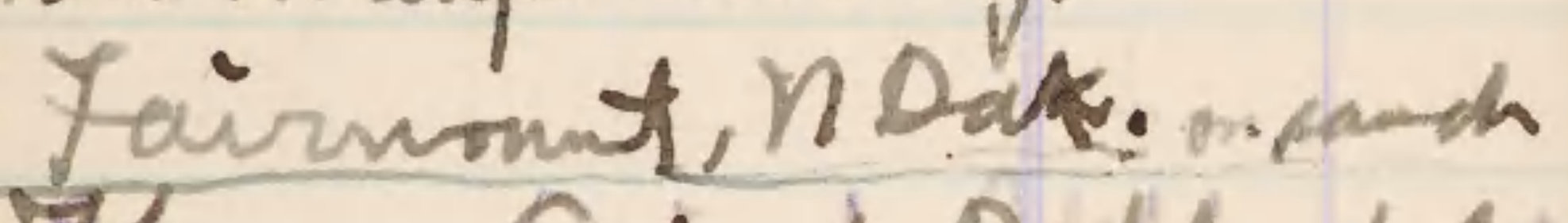

Thowes Siged Pritlland, or

Shopnomar Eaghenas

Juhias Tratum Richlavel

Edgar fohnson, Robriute

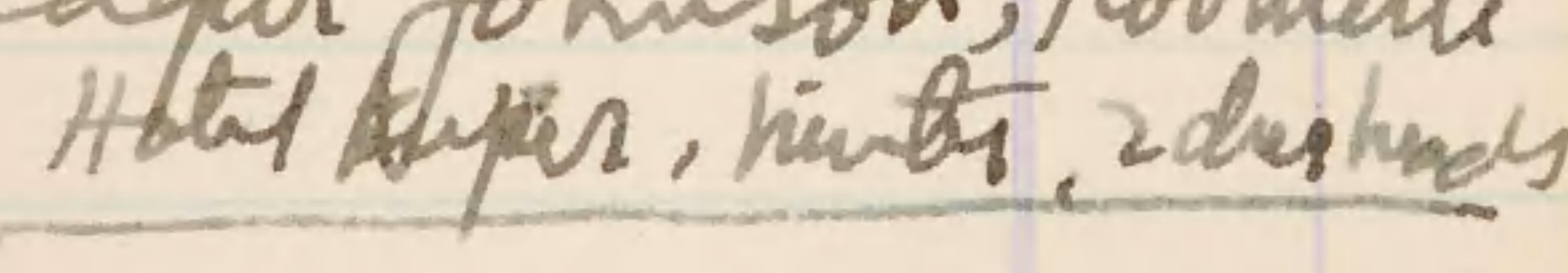





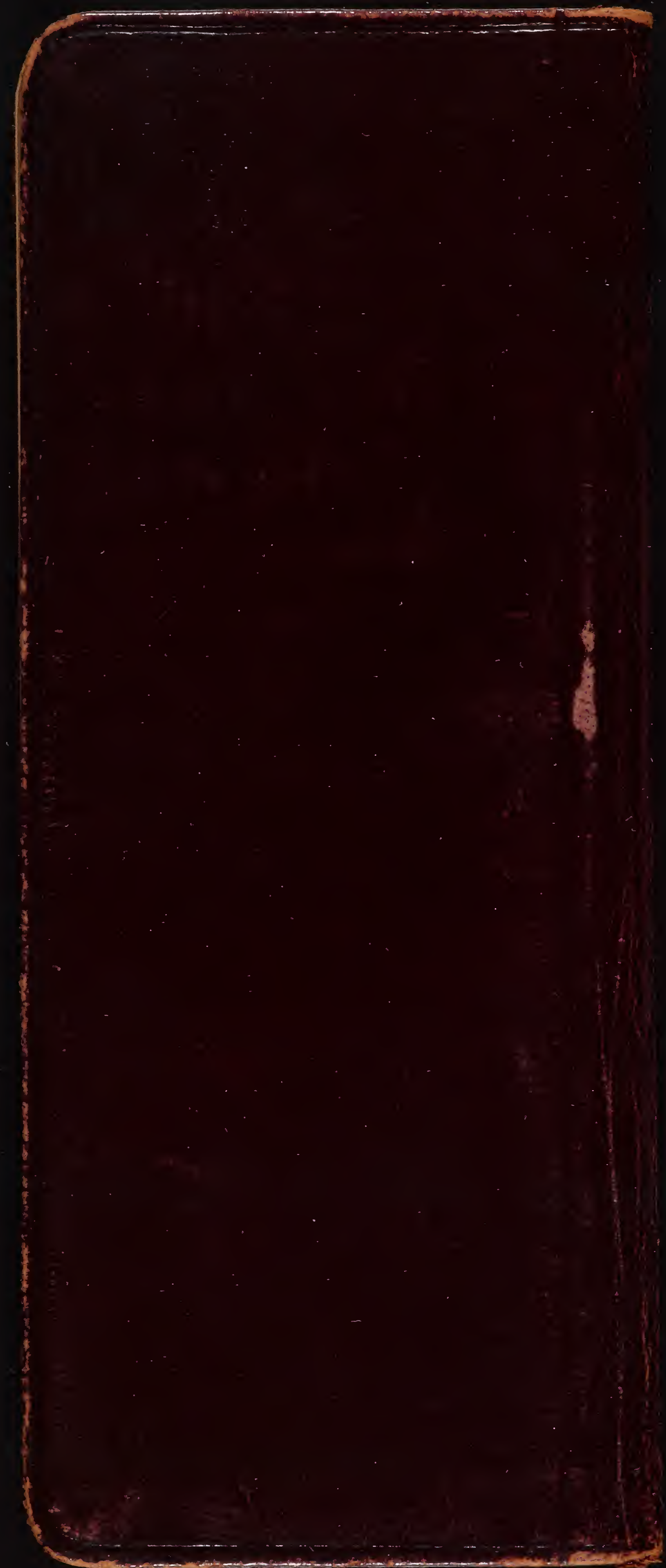

